\title{
Transmission spectra of highly irradiated extrasolar planet atmospheres
}

\author{
Dissertation \\ zur Erlangung des mathematisch-naturwissenschaftlichen \\ Doktorgrades \\ "Doctor rerum naturalium" \\ der Georg-August-Universität Göttingen \\ im Promotionsprogramm PROPHYS \\ der Georg-August University School of Science (GAUSS)
}

vorgelegt von

Lisa Nortmann

aus Hamburg

Göttingen, 2015 
Betreuungsausschuss

Prof. Dr. Stefan Dreizler

Sonnenphysik und Stellare Astrophysik, Institut für Astrophysik, Georg-AugustUniversität Göttingen, Germany

Prof. Dr. Ansgar Reiners

Sonnenphysik und Stellare Astrophysik, Institut für Astrophysik, Georg-AugustUniversität Göttingen, Germany

Mitglieder der Prüfungskommision

Referent: Prof. Dr. Stefan Dreizler

Sonnenphysik und Stellare Astrophysik, Institut für Astrophysik, Georg-AugustUniversität Göttingen, Germany

Korreferent: Prof. Dr. Artie Hatzes

Exoplaneten, Thüringer Landessternwarte Tautenburg, Germany

Weitere Mitglieder der Prüfungskommission:

Prof. Dr. Ansgar Reiners

Sonnenphysik und Stellare Astrophysik, Institut für Astrophysik, Georg-AugustUniversität Göttingen, Germany

Prof. Dr. Peter Hauschildt

Numerische Astrophysik, Hamburger Sternwarte, Germany

Prof. Dr. Wolfram Kollatschny

Extragalaktische Astrophysik, Institut für Astrophysik, Georg-August-Universität Göttingen, Germany

Prof. Dr. Wolfgang Glatzel

Sonnenphysik und Stellare Astrophysik, Institut für Astrophysik, Georg-AugustUniversität Göttingen, Germany

Tag der mündlichen Prüfung: 19.11.2015 


\section{Contents}

$\begin{array}{ll}\text { Abstract } & \text { iii }\end{array}$

$\begin{array}{lll}\text { Preface } & \text { v }\end{array}$

1 Introduction $\quad 1$

1.1 Exoplanet detection methods . . . . . . . . . . . . . . . . 1

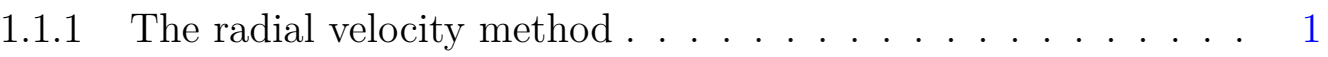

1.1.2 The transit method . . . . . . . . . . . . . . 2

1.2 Characterizing the planetary atmosphere . . . . . . . . . 5

1.2.1 Secondary eclipse and emission spectra . . . . . . . . . 5

1.2.2 Primary transit and transmission spectra . . . . . . . . . 7

1.3 Predictions of theoretical exoplanet models compared to previous work 9

1.4 This thesis . . . . . . . . . . . . . . . . . . 11

2 Investigation of the highly irradiated atmosphere of WASP-17b in the red optical with VLT/FORS2 13

2.1 Overview and motivation . . . . . . . . . . . . . . . . 13

2.2 Observations . . . . . . . . . . . . . . . . . . . . . . . . . . . . . . . . . . . . . .

2.3 Data reduction . . . . . . . . . . . . . . . . 15

2.3.1 Background estimation . . . . . . . . . . . . 15

2.3.2 Wavelength calibration and detector drift correction . . . . . . 15

2.3.3 Creation of light curves . . . . . . . . . . . . 16

2.4 The LADC - The origin of the systematic noise in FORS2 light curves 18

2.4.1 Occurrence of instrument systematics in previous spectroscopic FORS2 observations . . . . . . . . . . . . . . 21

2.4.2 Replacement of the FORS2 LADC prisms . . . . . . . . . . . 24

2.5 Analysis and results . . . . . . . . . . . . . . . 25

2.5.1 Limb darkening . . . . . . . . . . . . . . . . . 27

2.5.2 Modeling the instrument noise in the 'white light' curve . . . 27

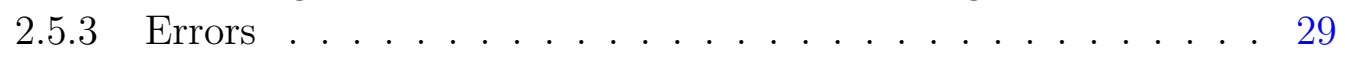

2.5.4 Red noise estimation . . . . . . . . . . . . . . . . . . 29

2.5.5 'White light' curve results . . . . . . . . . . . . . . . . . 31

2.5.6 Multi component noise signal . . . . . . . . . . . . . . 32

2.5.7 Transmission spectrum . . . . . . . . . . . . . 36

2.6 Comparison to literature data and theoretical models . . . . . . . . . 42

2.6.1 Rayleigh scattering . . . . . . . . . . . . . . 45 
2.6.2 Un-occulted star spots . . . . . . . . . . . . . . . 45

2.6.3 Third-light contamination . . . . . . . . . . 50

2.7 Conclusion . . . . . . . . . . . . . . . . . . . . . 50 50

3 Confirmation of the flat transmission spectrum of HAT-P-32Ab with GTC/OSIRIS \& Characterization of the M-dwarf companion HAT-P-32B

3.1 Paper: The GTC exoplanet transit

spectroscopy survey. IV. Confirmation of

the flat transmission spectrum of

HAT-P-32b . . . . . . . . . . . . . . . 53

3.2 Conclusion of the characterization of the

M-dwarf companion HAT-P-32B . . . . . . . . . . . . . . . 73

4 Summary, discussion and outlook $\quad 75$

4.1 Transmission spectra . . . . . . . . . . . . . . . . . . . . . . . . . . . . . . 75

4.2 Instrument systematics . . . . . . . . . . . . . . . . . . 76

4.3 Outlook on future observations with

VLT/FORS2 and GTC/OSIRIS . . . . . . . . . . . . . 77

$\begin{array}{ll}\text { Bibliography } & 79\end{array}$ 


\section{Abstract}

The ground-based facilities ESO/VLT+FORS2 (Very Large Telescope + FOcal Reducer and low dispersion Spectrograph) and GTC+OSIRIS (Gran Telescopio CANARIAS + Optical System for Imaging and low Resolution Integrated Spectroscopy) were used to probe the atmospheres of the two hot Jupiter planets WASP-17b and HAT-P-32Ab using multi-object-spectrophotometry. For WASP-17b, observed with FORS2, 14 transit light curves were extracted from the observed spectra and for HAT-P-32Ab, observed with OSIRIS, 20 light curves. Each light curve represents a $20 \mathrm{~nm}$ wide wavelength interval and was fitted with an analytical transit model to determine the wavelength dependent transit depth. For both planets strong nonastrophysical systematic noise, caused by the instrument, was found to affect the light curves. This thesis presents new findings regarding the source, nature and proper correction of these instrument specific and observing set-up dependent systematics, as well as the results for the planet transmission spectra. By including terms for the correction of the systematic noise signals into the light curve modeling, results for the transit depths were reached with uncertainties between 108 and 297 ppm for HAT-P-32Ab and between 127 and 481 ppm for WASP-17b.

The results for WASP-17b show a large-scale trend towards larger transit depth with shorter wavelength indicating strong absorption in the blue. Furthermore, these results act as a possible connection between two spectrophotometric studies in neighboring wavelength regions which are separated by a $>0.2 \%$ gap in transit depth. Neither a hot atmosphere containing titanium and vanadium oxide nor a cool atmosphere free of these molecules is fully consistent with the measurements. In contrast, a flat transmission spectrum was measured for HAT-P-32Ab, which could be indicative of a cloud layer at high altitude masking any atmospheric features below. Other possible explanations are an atmospheric depletion in alkali metals or a smaller than expected atmospheric scale height caused by either a lower planet equilibrium temperature or a heavier atmospheric mean molecular weight. The result is in excellent agreement with another ground-based study probing HAT-P-32Ab's optical transmission spectrum. This independent reproduction of compatible results demonstrates that ground-based measurements of exoplanet atmospheres can achieve robust and dependable measurements if the sources of systematic noise are well studied and corrected for appropriately.

Furthermore, an optical spectrum of the M-dwarf HAT-P-32B was obtained to correct its flux contribution to the HAT-P-32Ab transit light curves. By comparing the spectrum to PHOENIX stellar atmosphere models an effective temperature of $T_{\text {eff }}=3187_{-71}^{+60} \mathrm{~K}$ was derived. This value is slightly lower than results obtained by other groups in studies using broadband infrared data only. 



\section{Preface}

This thesis consists of four chapters. Chapter 1 is an introduction to the field of exoplanet research and the study of their atmospheres. Here the necessary background and motivation for the work presented in the remaining chapters is given. Chapter 2 presents the study of the transmission spectrum of the hot Jupiter planet WASP-17b. The text was taken from the manuscript 'Ground-based investigation of the highly irradiated atmosphere of the exoplanet WASP-17b in the red optical with VLT/FORS2' (Nortmann et al. 2015) written by me under consideration of minor editing suggestions by the co-authors. Co-authors are Stefan Dreizler, Jacob L. Bean and Travis Barman. All parts of the described analysis were done by me. The theoretical planet atmosphere models used for comparison to our data shown in Section 2.6 were calculated by Travis Barman. The manuscript was submitted to Astronomy \& Astrophysics for publication and has since been revised under critical reflection of comments given by the anonymous referee. The study was started prior to my PhD project. The planning and conduction of the observation of WASP$17 \mathrm{~b}$, the basic data reduction and extraction of spectra from the data described in Sections 2.2 to 2.3.2 were done as part of my Master project and contain several adopted sentences from my Master thesis 'Ground-based Spectroscopy of Exoplanet Atmospheres' handed in at the Georg-August-Universität Göttingen in 2011. In the Master thesis, further, a very preliminary analysis and feasibility study of the potential to extract useful information from this FORS2 data set was already reported on. The description of the observation and data reduction was again included in this dissertation as they provide essential information for the comprehension of the study conducted during my $\mathrm{PhD}$ project. My contributions to this study during the $\mathrm{PhD}$ make up the remainder of Chapter 2 (Section 2.3.3 to 2.7) and represent a comprehensive re-analysis of the data using of state of the art methods to evaluate the uncertainties and ascertain robustness of the results and a discussion of these results.

Chapter 3 presents the study of the transmission spectrum of the hot Jupiter planet HAT-P-32b (also known as HAT-P-32Ab) and also describes the study of the stellar companion HAT-P-32B. The majority of this chapter was published in Astronomy \& Astrophysics as the article 'The GTC exoplanet transit spectroscopy survey. IV. Confirmation of the flat transmission spectrum of HAT-P-32b' (Nortmann et al. 2016) after the final examination of this thesis and is reproduced in under Sect. 3.1; Credit: L. Nortmann, E. Pallé, F. Murgas, S. Dreizler, N. Iro and A. Cabrera-Lavers, A\&A, 594, A65, 2016, reproduced with permission OESO. The text of the article was written entirely by me with minor editing suggestions from the co-authors and the journal language editor. The co-authors are Enric Pallé, Felipe Murgas, Stefan 
Dreizler, Nicolas Iro and Antonio Cabrera-Lavers. All parts of the analysis were done by me. The theoretical planet atmosphere models used for comparison to our data were calculated by Nicolas Iro. The last subsection of the Chapter (Section 3.2), which presents a conclusion to the study of the stellar companion HAT-P32B, was not included in journal publication. The final Chapter 4 summarizes and discusses the general results of this thesis and outlines future prospects. 


\section{Chapter 1}

\section{Introduction}

We live in exciting times. The possible existence of foreign worlds beyond our Solar System has been subject of philosophical debate for thousands of years. For several decades, now, they have been depicted in colorful detail in science fiction media. Actual scientific evidence for the presence of such worlds, however, had not been found until a little over 20 years ago. It was in 1992 when the first confirmed extra solar planet detection was reported by Wolszczan \& Frail. The planet, orbiting the millisecond pulsar PSR1257+12, was found by detecting the projected movement of the host star around the common center of gravity of the starplanet system. This was done by measuring the very small timing irregularities in the pulsation caused by the variations in light travel time. This detection method was limited to pulsating stars, which expose their planets to a very extreme environment considerably different from our own Solar System. This might be the reason why it was only after the first planet was found around a solar-like star in 1995 by Mayor \& Queloz that the field of exoplanets truly gained momentum. Just like the detection of PSR1257+12b the presence of this planet, Peg 51b, was detected indirectly by measuring the host star's movement along the line of sight of the observer using a Doppler shift of its spectral lines. This method, called the radial velocity method, is still one of the most successful detection methods for exoplanets to date only outperformed by the transit method.

\subsection{Exoplanet detection methods}

\subsubsection{The radial velocity method}

Starting out as a method to characterize binary star orbits, the radial velocity (RV) method today is one of the most successful exoplanet detection methods with 470 candidates confirmed. ${ }^{1}$ In this method the existence of a planet is indirectly inferred by the movement of the star. Due to the presence of one or more planets the common center of gravity is shifted from the stellar center. All objects in the system including the host star orbit the barycenter. As the system is not always aligned with the observer's line-of-sight, the stellar movement can be broken down in its part along

\footnotetext{
${ }^{1}$ www.exoplanets.org (status as of 9 th October 2015)
} 
the line-of-sight and the part perpendicular to that direction. The stellar movement in the line-of-sight direction can be inferred from changes in the star's velocity. This in turn can be measured by monitoring the spectral lines of the star and detecting small bluewards and redwards shifts caused by the Doppler effect.

The observed shift in observed wavelength $\Delta \lambda$ of a light emitting object with a non-zero velocity relative to the observer is given by Eq. 1.1.

$$
\Delta \lambda=\frac{v}{c_{0}} \lambda_{0}
$$

where $\lambda_{0}$ is the wavelength emitted by the object, $c_{0}$ is the velocity of light ${ }^{2}$ and $v$ the velocity of the star relative to the observer. This equation is only correct fot small velocities i.e. $v \ll c_{0}$. The relation between the measurable velocity amplitude $K$ and the physical planet and system parameters is given in Eq. 1.2, which is adopted from (Cumming et al. 1999, Equation 1). In Eq. $1.2 M_{\mathrm{p}}$ is the planetary mass, $M_{\star}$ the mass of the host star, $P$ is the orbital period, $e$ the eccentricity of the planet orbit and $G$ the gravitational constant ${ }^{3}$.

$$
K=\left(\frac{2 \pi G}{P}\right)^{1 / 3} \frac{M_{p} \sin i}{\left(M_{p}+M_{\star}\right)^{2 / 3}} \frac{1}{\sqrt{1-e^{2}}}
$$

The method is strongly aided by stellar spectral types that exhibit many well separated lines and by large planetary masses. As the observer can only measure the projected movement of the star, the inclination $i$ of the system to the observers line-of-sight introduces an uncertainty if it is unknown. In that case only a lower limit for the mass of the planet can be determined. At inclinations close to zero, i.e. when the system is observed almost face on, a large portion of the stellar movement would be perpendicular to the line-of-sight of the observer. In such cases, however, the displacement of the star on the night sky can sometimes be large enough to be detected by astrometric measurements. These are often conducted as follow up of RV detections to determine upper limits on the planetary masses (Benedict et al. 2002, 2006, McArthur et al. 2004).

In the opposite case, if the inclination of the system instead is close to $90^{\circ}$, the probability rises that the planet can be seen transiting in front of its host star.

\subsubsection{The transit method}

The currently most successful exoplanet detection method with 1147 detections to date $^{4}$ is the transit method. The large number of detections is still constantly increasing, a fresh supply of data being provided by several ground based surveys (e.g. SuperWASP, HATNet, TrES) and, until recently, by two space missions CoRoT and Kepler, all dedicated to the detection of transiting planets. During a transit the planet passes in front of it host star in the line of sight of the observer, causing a decrease in the total measured stellar flux. If the star is approximated as a

\footnotetext{
${ }^{2} c_{0}=299792458\left[\mathrm{~m} \mathrm{~s}^{-1}\right]$

${ }^{3} G=6.67408 \pm 0.00031 \cdot 10^{-11}\left[\mathrm{~m}^{3} \mathrm{~kg}^{-1} \mathrm{~s}^{-2}\right]$

${ }^{4}$ www.exoplanets.org (status as of 9th October 2015)
} 
homogeneously emitting disc and the planet as a completely dark disk the observable drop in observed flux $F$ or so called transit depth $\delta \equiv \Delta F$ is proportional to the planet-to-star area ratio as given in Eq. 1.3 where $R_{\mathrm{p}}$ is the planet radius, $R_{\star}$ the stellar radius.

$$
\delta=\left(\frac{R_{\mathrm{p}}}{R_{\star}}\right)^{2}
$$

As a consequence the planet radius in units of the stellar radius can be inferred from the measured transit depth. In the early days a majority of the planets found with this method were Jupiter sized planets orbiting their host star in very close orbits. As these planets are receiving a lot of external radiation from their host star they are generally referred to as hot Jupiters. Their detection was further favored by the fact that for a homogeneous distribution of inclinations the probability $p$ to observe a transit increases for smaller orbital distances as given in Eq. 1.4 where $a_{\mathrm{p}}$ is the semi-major axis of the planet orbit.

$$
p=\frac{R_{\star}}{a_{\mathrm{p}}}
$$

Another factor is that the close distance to their host stars result in very short orbital periods, leading to the transit signal repeating with a frequency of mere days. Opposed to this, a transit of our Solar System's Jupiter would only be observable to an outside observer every 11.86 years. With the observation of several transit events the period $P$ can be determined. The inclination of the planet's orbit $i$, its semi-major axis in units of the stellar radius $a_{\mathrm{p}} / R_{\star}$ and the host star's density $\rho_{\star}$ can be derived when combining the measurements of the transit depth $\delta$, total transit duration $t_{\mathrm{T}}$ (including ingress and egress), duration of the transit bottom $t_{\mathrm{B}}$ (excluding ingress and egress) and orbital period $P$ (for details see Equation 8, 9 and 13 in Seager \& Mallén-Ornelas 2003). The transit method is however not limited to extreme hot worlds. The very successful space missions CoRoT and Kepler have found a multitude of planets. The Kepler survey has even yielded forward a sub-Mercury sized planet (Barclay et al. 2013).

As several constellations can mimic a transit light curve (e.g. a blend by a background binary) RV measurements are desired to additionally determine the planetary mass and thus confirm the planetary nature of an object (Santerne et al. 2013). While this can be routinely done for large gas giants it is still problematic for lighter planets (and faint host stars) due to limits in the precision reachable with current RV instrumentation. As many planet candidates detected by the space missions Kepler and CoRoT fall into this category, instead of a confirmation of the planetary nature of a candidate the concept of planet validation was brought forward. In this concept a planet is 'validated' if the probability of the planetary nature of the object significantly outweighs the sum of the probabilities of all possible false positive scenarios (Díaz et al. 2014).

\subsubsection{Characterizing the planetary system}

The charm of the transit method does not only lie in the detection of planets, but also in the opportunity that the observation of a transit event gives us to further 
characterize the system.

Small variations in the mid-transit-time of the periodically reoccurring transit event for instance can reveal additional objects in the system perturbing the transiting planets orbit (Ballard et al. 2011). Such transit timing variations (TTV) in combination with transit duration variation (TDV) measurements could in the future lead to the detection of an exomoon (Kipping 2009). TTVs have also been used as means to confirm the planetary nature of Kepler candidates by deriving planetary masses out of reach of RV precision e.g. in the case of the multiplanetary systems Kepler-9 (Holman et al. 2010).

Another benefit of transiting planets is that the spin-orbit alignment of the system can be determined due to the Rossiter-McLaughlin effect when the RV signal of the host star is observed during a planetary transit (Ohta et al. 2005, Winn et al. 2005, 2006). As the star rotates around its own axis, one side will move towards and the other side will move away from the observer. This rotation leads to Doppler broadening of the stellar lines. During transit the planet consecutively occults parts of different velocity of the star, resulting in a distortion of the stellar line profiles. When measuring the RV signal this distortion mimics a change in RV velocity. The resulting signal reveals the path of the plenary disc over the rotating stellar surface and, thus, the system alignment.

Using the planetary disc as a means to sample different regions of a star can further reveal inhomogeneities in the brightness of the stellar disc. Deviating from the simplified model of a homogeneously emitting stellar disc, where the transit depth is given by Eq. 1.3, a real host star will show limb-to-center brightness variations and in some cases dark star spots and bright plages that influence the actual measurable drop in brightness.

\subsubsection{Stellar limb darkening}

The stellar disk shows center to limb brightness variations because, at the limb the line of sight penetrates the stellar sphere at an oblique angle. The photons stemming from an optical depth of $\tau=1$ are at the limb, and therefore, produced in a much higher layer of the atmosphere than at the center of the projected stellar disc. As in most cases the temperature drops with increasing stellar radius, therefore the photons reaching the observer from the limb of the stellar disc are produced at a colder environment. This limb darkening i.e. the wavelength dependent drop in specific intensity from the center $I_{\lambda}(1)$ towards the limb is often described by a linear (Eq. 1.5) or a quadratic law (Eq. 1.6) (Kopal 1950).

$$
\begin{gathered}
I_{\lambda}(\mu)=I_{\lambda}(1) \cdot\left[1-u_{1}(1-\mu)\right] \\
I_{\lambda}(\mu)=I_{\lambda}(1) \cdot\left[1-u_{1}(1-\mu)-u_{2}(1-\mu)^{2}\right]
\end{gathered}
$$

where $I_{\lambda}(\mu)$ is the specific intensity, $\mu=\cos (\vartheta)$, with $\vartheta$ being the angle between the line of sight and the surface normal, and $u_{1}$ and $u_{2}$ the linear and quadratic limb-darkening coefficients.

The effect of a limb darkened star on a transit light curve manifests in a curvature of the transit bottom. It is important to note that in these cases a large fraction 
of the total observable stellar flux is emitted from the center of the stellar disc and the maximal transit depth, reached when the planet crosses over this area, is deeper than it would have been for a homogeneously emitting sphere. Therefore, it is crucial to consider limb darkening carefully when modeling transit light curves, especially when accurate measurements of the transit depth are desired.

\subsubsection{Stellar spots and plages}

Similar to center-to-limb brightness variations stellar spots and plages cause an inhomogeneous stellar surface. As a consequence their existence will influence the measured transit depth, even if the planet does not cross them during transit. An unocculted bright plage will make the transit appear shallower, as a lower percentage of the total emitted flux is obscured by the planet. Similarly, an un-occulted dark spot will cause a deeper transit. In case the planet crosses over a spot or a plage this often can be seen as a upwards or downwards anomaly in the transit light curve. These effects can, however, also be more subtle and be mistaken for red-noise. Therefore, planet host stars are often monitored for photometric variability associated with spots and plages (Sing et al. 2011b, Dragomir 2015).

\subsection{Characterizing the planetary atmosphere}

When the radius and mass of a planet have been obtained via transit and RV measurements its bulk density can be calculated. However, this information is not always enough to determine a planet's nature as different internal compositions would yield identical average densities. This can, for instance, make it impossible to distinguish between a planet with a heavy core and a thin hydrogen or helium atmosphere and an ocean planet with a water-ice core (Adams et al. 2008). This degeneracy can only be broken by measuring this planet's atmosphere highlighting the importance of such measurements.

Furthermore, the atmosphere of a planet is the only way to obtain any insight on its actual habitability. Any chance of detecting life outside of our Solar System in the future will have to rely on the remote measurement of atmospheric biomarkers (Seager \& Deming 2010).

Current atmospheric studies of exoplanets are heavily benefitting from the geometry of transiting planets.

\subsubsection{Secondary eclipse and emission spectra}

If the eccentricity of a transiting planet's orbit is moderate then not only the occultation of the star by the planet, but also the occultation of the planet by the star can be measured. Compared to the star the planet is very faint in the optical, but the contrast becomes more favorable in the infrared where most successful secondary eclipse detections were reported (e.g. Croll et al. 2011, Zhou et al. 2014). When approximating the planet as a black-body emitter, the peak of the planetary emission increases and moves towards shorter wavelength with rising planetary equilibrium temperatures. This has made it possible to detect secondary eclipses in the optical 
for very hot planets like CoRoT-2b (Alonso et al. 2009, Snellen et al. 2010). In the optical disentangling the signal of light emitted from the planet and contributions of reflected star light becomes difficult.

If the secondary eclipse is observed in several broad bands or, even better, at higher resolution the dayside temperature can be obtained and molecular features in the emission spectrum can be detected (e.g. Knutson et al. 2008, 2009, Fressin et al. 2010).

A planet's equilibrium temperature $T_{\text {eq }}$ is expected to increase with its proximity to its host star due to the external irradiation. However, the planetary albedo $A$ and its efficiency in re-distributing heat e.g. from the day side to the night side also play a major role. An estimate for $T_{\text {eq }}$ can be calculated with Eq. 1.7, where $T_{\text {eff }}$ is the host stars effective temperature and the heat-redistribution is considered by introducing the factor $f$ which assumes the value $f=1 / 4$ for fully efficient redistribution over the whole planetary surface and larger values for more inefficient redistribution of planets causing large contrasts between the day and night side.

$$
T_{\text {eq }}=T_{\text {eff }}(f(1-A))^{1 / 4} \sqrt{\frac{R_{\star}}{a_{\mathrm{p}}}}
$$

The heat-transport is especially interesting for close-in planets as they are often tidally locked, i.e. always face their host star with the same side. Depending on the efficiency of energy re-distribution, the terminator temperatures probed by transmission spectroscopy (see Sect. 1.2.2) consequently could differ strongly from the temperatures at the sub-stellar point on the planet's day side probed during secondary eclipse measurements. If a strong contrast exists between the night and day side, a variation in planet emission can be observed when a full phase curve of a planet orbit is measured. Using the secondary eclipse and phase curve observations Knutson et al. (2007) were able to detect a slight offset of the brightest point of the hot Jupiter HD 189733b from the sub-stellar point, implying the existence of a heat-transporting mechanism. A similar asymmetry the phase curve was also found by Stevenson et al. (2014c) who obtained color resolved phase curve observations of unprecedented precision for the transiting planet WASP-43b. These measurements allowed the authors to determine the longitude resolved thermal structure of the planet's atmosphere.

Direct detection of exoplanet emission without the aid of a secondary eclipse at low to medium resolution could so far only been achieved for a few young and bright planets at large separations to their host stars (Barman et al. 2015). However, using very high resolution $(\sim 100000)$ spectroscopy makes it possible to trace the Doppler shifted lines of the planet and detect them separated from host star emission and telluric absorption lines. One example for the use of this method is the successful detection of the Doppler shifted CO lines of the planet $\tau$ Boötis b by Brogi et al. (2012) using the ground-based instruments VLT/CRIRES.

For planets that do transit their host stars, however, insights into the exoplanet atmosphere can not only be gained from the secondary eclipse but also from the primary transit. 

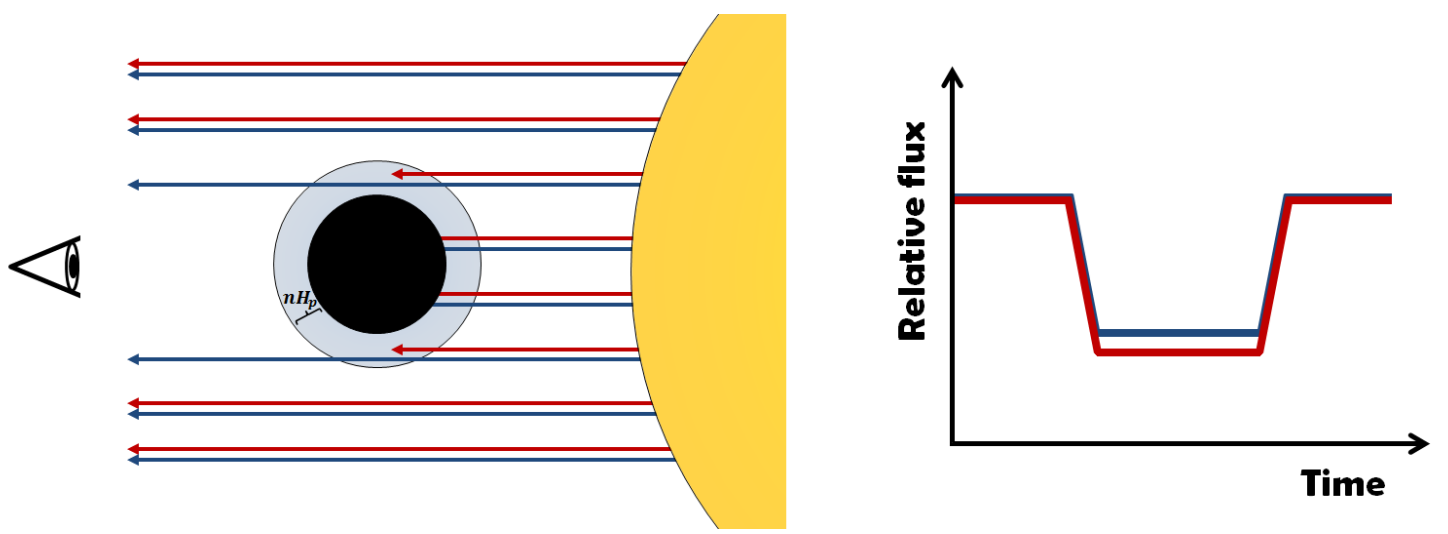

Figure 1.1: Left panel: sketch of the geometry during transmission spectroscopy of a transiting planet. The depicted atmosphere is opaque for 'red' photons and transparent for 'blue' photons. Right panel: transit light curve in the depicted two colors. The measured transit depth is deeper in the red than in the blue by approximately $\Delta \delta$ (Eq. 1.8).

\subsubsection{Primary transit and transmission spectra}

During the primary transit part of the stellar light can pass through the upper layers of the atmosphere. Only in certain regions, where the constituents in the atmosphere cause it to be optically thick, the star light will be blocked, letting the planet appear larger in these wavelength regions. The transmission spectrum of a planet's atmosphere can therefore be directly inferred from measurements of its wavelength dependent transit depth (Seager \& Sasselov 2000). The expected signal is small even for the most favorable candidates (between $0.1-1 \mathrm{mmag}$ ). An estimate of the amplitude of an atmospheric absorption signal, which can extend over several $(n)$ atmospheric scale heights $H_{\mathrm{p}}$ (see Eq. 1.9), can be calculated with Eq. 1.8.

$$
\begin{gathered}
\Delta \delta=\frac{\left(R_{\mathrm{p}}+n H_{\mathrm{p}}\right)^{2}}{R_{\star}^{2}}-\frac{R_{\mathrm{p}}^{2}}{R_{\star}^{2}}=\frac{2 R_{\mathrm{p}} n H_{\mathrm{p}}+\left(n H_{\mathrm{p}}\right)^{2}}{R_{\star}^{2}} \\
H_{\mathrm{p}}=\frac{k_{b} T_{\mathrm{eq}}}{\mu g_{\mathrm{p}}}
\end{gathered}
$$

where $T_{\text {eq }}$ is the planet equilibrium temperature, $g_{\mathrm{p}}$ the planetary gravitational acceleration, $m$ the mean molecular weight of the atmosphere and $k_{B}$ the Boltzmann constant. As the transmission feature signal increases with the planet radius and its scale height, which in turn depends on the planet's equilibrium temperature, many of the best candidates of exoplanet atmosphere studies are highly irradiated giant (hot Jupiter) planets. A notable exception is the Super-Earth GJ 1214b, which closely orbits an M-dwarf, thus, exhibiting a large planet-to-star radius ratio due to the small radius of the star, rather than the large radius of the planet (Charbonneau et al. 2009). Observations of targets with large expected signals are the perfect basis to test and improve our observing strategies and find the limiting factors in our instruments, thus, paving the way to maybe one day detecting bio-signatures in terrestrial exoplanet with significantly smaller scale heights and consequently significantly smaller signals. 


\subsubsection{Space-based Observation}

The first successful measurement of an exoplanet transmission spectrum was achieved from space by Charbonneau et al. (2002) using the Space Telescope Imaging Spectrograph (STIS) of the Hubble Space Telescope (HST). Charbonneau et al. detected an excess absorption in the Na D lines when observing the hot Jupiter HD 209458b during transit in the wavelength region between 581.3-638.2 nm. HST instrumentation has since enabled researchers to probe the transmission of planet atmospheres in a wide range of wavelengths. Many of these studies made use of STIS, which alone offers an impressive coverage of the optical $(115-1000 \mathrm{~nm})$. But also other HST instruments were employed e.g. the Near Infrared Camera and Multi-Object Spectrometer (NICMOS, $800-2500 \mathrm{~nm}$ ), which was in operation till 2008 and the infrared channel $(800-1700 \mathrm{~nm}$ ) of the Wide Field Camera 3 (WFC3), which was mounted on HST in 2009 (e.g. Berta et al. 2012, Nikolov et al. 2014, 2015, Sing et al. 2015). In addition to HST, the Spitzer Space Telescope, in particular its Infrared Array Camera (IRAC) offering simultaneous imaging in four channels $(3.6 \mu \mathrm{m}, 4.5 \mu \mathrm{m}$, $5.8 \mu \mathrm{m}$ and $8 \mu \mathrm{m}$ ), was used for secondary eclipse measurements (e.g. Charbonneau et al. 2008, Knutson et al. 2008, Todorov et al. 2010). After the liquid helium supply was depleted in 2009 only the two channels $3.6 \mu \mathrm{m}$ and $4.5 \mu \mathrm{m}$ remained operable. Despite the fact that space offers a rather stable environment reducing possible external noise sources the data obtained with HST/NICMOS and Spitzer/IRAC was found to be strongly affected by systematics. Their proper correction and the derived results for planet atmospheres were under strong debate in the community (Gibson et al. 2011, Diamond-Lowe et al. 2014). The main drawback of space-based observations, however, is that the available mirror sizes are small compared to that of ground-based facilities. The launch of the James Web Telescope (JWST ${ }^{5}$ ) planned in 2018 has a chance to remedy this disadvantage with its primary mirror size of $6.5 \mathrm{~m}$. JWST planned wavelength coverage $(600-2800 \mathrm{~nm})$, however, will miss out entirely on the blue optical, which, once HST eventually reaches its inevitable end of mission, will only accessible by ground-based studies.

\subsubsection{Ground-based Observation}

When choosing to observe from the ground the available mirror sizes i.e. collecting area of the available facilities increases significantly. However, other obstacles arise from the Earth's own atmosphere. In order to correct for effects of atmospheric variations on the data a reference star can be observed together with the planet host star. Division by the reference star flux should then yield data free from telluric variations. This works well for imaging data and has been used to probe planet spectra in a few distinct band passes using either broadband filters or very narrow tunable filters (e.g. Bento et al. 2014, Sing et al. 2011a, Murgas et al. 2012). However, when using spectroscopy with a narrow spectroscopy slit even very small misalignments between the two observed stars can lead to drastic differences in slit flux losses, especially if the seeing is variable during the observations. To avoid any slit losses one can use slit-less spectroscopy, or instead use very broad slits in which

\footnotetext{
5 jwst.nasa.gov
} 
the entire point spread function (PSF) of the stars fit. The latter is preferable over the first, as it helps avoid excessive sky background flux and contamination of the desired spectra by nearby stars. The use of broad slitlets was pioneered for ground-based exoplanet transmission studies by Bean et al. (2010) observing the Super-Earth GJ 1214b at the Very Large Telescope facility (VLT) using the FOcal Reducer and low dispersion Spectrograph 2 (FORS2) with a spectroscopy mask. Following their success the method was employed at several other facilities, either with spectroscopy masks enabling the measurements of multiple reference stars or by using one long and broad-slit, inside which usually only two stars can be aligned. One pitfall of this method is that the required data quality can only be reached if a suitable reference star of similar brightness and color is available at a very close distance to the planet host star. While this limits the list of potential targets, ground based transmission spectroscopy studies have been very successful e.g. with GTC/OSIRIS (Sing et al. 2012, Murgas et al. 2014, Mallonn et al. 2015), Magellan/IMACS (Jordán et al. 2013) and Gemini/GMOS (Gibson et al. 2013a,b).

\subsection{Predictions of theoretical exoplanet models compared to previous work}

Early theoretical models for transiting extrasolar giant planets by Seager \& Sasselov (2000) predicted strong alkali absorption lines (Na I and K I resonance doublets) and a Rayleigh slope in the optical spectra of their atmospheres. These models were assuming the planetary atmospheres to be similar to those of L dwarfs, as these exhibit comparable temperatures. In their work Seager \& Sasselov already mention the effect of cloud layers, pointing out, that depending on their height in the atmosphere they could block out parts of the atmospheric absorption features.

Seager \& Sasselov indicate that the depth to with the observation has penetrated the planet, could be inferred from an investigation of the measured line wings of the alkali doublets, as these should get progressively wider at greater depths due to pressure broadening.

Over time, theoretical models were refined by adding opacity sources (e.g. opacity tables by Freedman et al. 2008) and by considering atmospheric circulation (e.g. Burrows et al. 2010). Three-dimensional models were realized by creating latitudinal and longitudinal grids of vertical one-dimensional columns. In these models each one-dimensional column exhibited an individual temperature-pressure profile from which corresponding distinct absorption cross sections were calculated under assumption of local chemical equilibrium (Fortney et al. 2010). Possible effects of non-equilibrium chemistry, for instance driven by photochemistry processes on the highly irradiated day side and non-gaseous opacities of clouds and hazes were neglected.

The models published by Fortney et al. (2010) predicted strong $\mathrm{H}_{2} \mathrm{O}$ and $\mathrm{CO}$ absorption bands in the infrared transmission spectra of highly irradiated giant planet atmospheres. For cooler atmospheres instead of $\mathrm{CO}$ the model infrared spectra started exhibiting broad $\mathrm{CH}_{4}$ absorption features. When the modeled planets were exposed to strong stellar irradiation their optical transmission spectra differed from 
the early model spectra presented by Seager \& Sasselov (2000). Instead of showing alkali line absorption their optical transmission spectra were dominated by broad titanium and vanadium oxide absorption bands. Titanium oxide ( $\mathrm{TiO}$ ) and vanadium oxide (VO) are found in cool stellar atmospheres and therefore were expected to play an important role in exoplanet atmospheres as well.

Fortney et al. (2008) proposed two different classes of hot Jupiters, based on their received irradiation and the metal oxide content of their upper atmospheres. In his hypothesis the strong irradiation by their host stars would create temperatures in which $\mathrm{TiO} / \mathrm{VO}$ could exist in the oxidized form. As these metal oxides show absorption over large parts of the optical they could heat up and facilitate the formation of a thermal inversion layer i.e. a region in which the atmospheric temperature rises with increasing altitude and decreasing atmospheric pressure. Fortney et al. stated that the occurrence of inversion layers would be strongly linked to the received stellar irradiation and, thus, the proximity of the planet to its host star. The first proposed class, the 'pM' planets, would be the highly irradiated giant planets, with an inversion layer and an optical transmission spectrum dominated by TiO. The second class, the 'pL' planets, would exhibit no thermal inversion and their optical spectra would exhibit absorption by the $\mathrm{Na}$ and $\mathrm{K}$ doublets. The transition zone between these two classes was estimated to occur at an orbital distance of $\sim 0.04-0.05 \mathrm{AU}$.

This hypothesis was challenged by observations as several highly irradiated close-in gas giants did not exhibit the predicted inversion layer (Gillon et al. 2010, Fressin et al. 2010, Machalek et al. 2008). Also none of the planets, whose optical transmission was studied showed solid evidence for $\mathrm{TiO} / \mathrm{VO}$ absorption, with only one report of a tentative detection in the literature for the hot Jupiter HD 209458b (Désert et al. 2008). HD 209458b was considered to show clear signs of an inversion layer (Knutson et al. 2008). This results, however, has recently been contradicted by reports of little to no evidence for a thermal inversion from a re-analysis of Spitzer data by Diamond-Lowe et al. (2014) and new ground-based observations (Schwarz et al. 2015). Madhusudhan \& Seager (2010) suggested that the inference of thermal inversions in hot Jupiter atmospheres could be biased by the choice of chemical compositions in the assumed model atmosphere. Madhusudhan (2012) provided planet atmosphere models with varying $\mathrm{C} / \mathrm{O}$ ratios and suggested that a depletion of $\mathrm{TiO} / \mathrm{VO}$ could be driven by $\mathrm{C} / \mathrm{O}$ ratios larger than 1 , which would cause all available oxygen to be bound in $\mathrm{CO}$. Other explanations for the lack of $\mathrm{TiO} / \mathrm{VO}$ are given by Spiegel et al. (2009) who propose that these heavy molecules would settle and rain out in deeper, colder layers of the atmosphere from where they could only be reintroduced into higher layers by very strong vertical mixing. Parmentier et al. (2013), further, mention a cold night side as a possible cold-trap for TiO/VO. Knutson et al. (2010) discuss stellar activity as a possible hindrance for the formation of thermal inversions layers. They suggest that UV irradiation could photo-dissociate the species that would otherwise heat up the atmosphere.

After several years of data collection and analysis, observed transmission spectra show several distinct re-occurring features unanticipated by models. For instance, instead of the predicted hot $\mathrm{TiO} / \mathrm{VO}$ absorption dominated and cold alkali metal absorption dominated atmospheres many transmission spectra were actually found 
to be completely featureless. The most prominent example for such a completely flat spectrum is the well-studied Super-Earth GJ 1214b (Bean et al. 2010, 2011, Berta et al. 2012, de Mooij et al. 2013, Kreidberg et al. 2014), but several similar cases have been reported for other planets (e.g., Gibson et al., 2013a (WASP-29b), Gibson et al., 2013b (HAT-P-32b); Knutson et al., 2014 (GJ 436b)). These flat spectra can be explained by high altitude cloud layers blocking the star light and making a detection of species in lower atmospheric layers impossible. In addition to clouds, also hazes have been found to play a major role in the optical. While other explanations of an increasing planet radius towards shorter wavelength, like the crossing of stellar pages during transit (Oshagh et al. 2014), have been discussed, Rayleigh and haze scattering are still considered the most likely culprits. For some planets the steep featureless slopes associated with Rayleigh and haze scattering are the only feature seen in their transmission spectra e.g. HD 189733b (Sing et al. 2011b) and Tres-3b (Parviainen et al. 2016). Other planets exhibit narrow alkali line absorption features on top of such slopes. This is the case for WASP-6b, for which in addition to a haze slope (Jordán et al. 2013) a tentative detection of the Na and K line cores was reported by Nikolov et al. (2015). WASP$31 \mathrm{~b}$ does not only feature a Rayleigh slope and $\mathrm{K}$ absorption, but also a muted water band in the infrared, suggesting a cloud deck masking lower layers of the atmosphere (Sing et al. 2015). The detection of a strong K but weak Na absorption indicates a sub-solar $\mathrm{Na} / \mathrm{K}$ abundance ratio for this planet. The authors state that this finding might point to non-solar metal distributions during formation or by later condensation of Na.

For several planets successful detection of the infrared water band at $1.1-1.7 \mu \mathrm{m}$ was reported based on HST/WFC3 observations e.g. for WASP-12b, WASP-17b, WASP-10b (Mandell et al. 2013), XO-2b, HD 209458b (Deming et al. 2013) and HAT-P-1b (Wakeford et al. 2013). For HAT-P-1b a large offset between the optical HST/STIS data and the infrared HST/WFC3 data was found by Nikolov et al. (2014). This offset could not explained by regular models. The authors speculate about a hypothetical high opacity absorber which is only efficient in the optical and rule out stellar activity and non-linearity effects of the WFC3 instrument as possible error sources. Not discussed was the possibility of the infrared data being affected by the subtle wavelength independent visit-long time-dependent systematic which was found to appear in WFC3 data by Stevenson et al. (2014a). This systematic, if not taken into account, could lead to systematically offset transit depth measurements.

\subsection{This thesis}

Many of the space-based and ground-based studies report non-astrophysical systematic noise in their data. Often these systematics are instrument specific and not well understood. Several approaches to their proper correction have been discussed, including modeling the systematics as functions of auxiliary parameters or as Gaussian processes and attempting to disentangle them from the astrophysical signal using independent component analysis (Gibson et al. 2012, Gibson 2014, Thatte et al. 2010, Waldmann 2012). As an (intermediate) outcome, results are still considered 
with skepticism and their robustness is questioned.

In order to test and refine existing theoretical models for exoplanet atmospheres, reliable observational data and continuous wavelength coverage is needed. Furthermore, to pave the way for future observations of terrestrial planet atmospheres we have to understand the limits of our instruments and find ways to overcome them. In this thesis I present ground-based observations of the two hot Jupiters WASP17b (Chap. 2) and HAT-P-32Ab (also commonly known as HAT-P-32b, Chap. 3) obtained in the optical at two different facilities. In the case of WASP-17b, observed at the VLT with FORS2, the observations cover a previously unrobed wavelength region closing the gap between the two previous studies of Mandell et al. (2013) and Bento et al. (2014). In the case of HAT-P-32Ab, observed with OSIRIS at the Gran Telescopio CANARIAS (GTC), the wavelength region covered by the observations was previously studied by Gibson et al. (2013b). Here, our contribution lies in the independent repetition of the measurements. It represents a necessary exercise, aiming to establish whether robust results have and can be obtained despite the existence of strong correlated noise in the data. We found such systematic noise to affect both our data sets. I address the findings about their sources in this thesis as information of this kind can help to improve the design of the next generation of instruments aiming at the characterization of rocky and potentially habitable worlds. 


\section{Chapter 2}

\section{Investigation of the highly irradiated atmosphere of WASP-17b in the red optical with VLT/FORS2}

All Sections of this chapter were taken from the paper manuscript 'Ground-based investigation of the highly irradiated atmosphere of the exoplanet WASP-1'b in the red optical with VLT/FORS2' (Nortmann et al. 2015). In the manuscript the first section of this chapter (Sect. 2.1) is part of the introduction section. The phrase 'this paper' was changed to '[this chapter]' to clarify the intended reference.

\subsection{Overview and motivation}

The hot Jupiter WASP-17b was discovered in 2010 and has a retrograde orbit around its host star, a F6 star with sub-solar metallicity (Anderson et al. 2010). The planet is heavily irradiated, which leads to an estimate of the dayside equilibrium temperature equal to $1755 \pm 28 \mathrm{~K}$ (Southworth et al. 2012) when bond albedo and heat re-distribution are neglected. With a density of $\rho=0.0618 \pm 0.0051 \rho_{\text {Jup }}$ (Southworth et al. 2012) it is one of the least dense planets known today. This, together with its high equilibrium temperature and allover very deep transit signal, makes it a prime target for transmission spectroscopy. As a consequence, WASP-17b has been observed by several others and has been successfully probed for sodium absorption by Wood et al. (2011) and Zhou \& Bayliss (2012). Recent publications of Bento et al. (2014) showed the results of multicolor broadband photometry in the SDSS $u$ ' $g$ ' and $r$ ' band, i.e. probing the range from 325 to $690 \mathrm{~nm}$ divided in three sub-regions. Furthermore, Mandell et al. (2013) provided narrowband (28 $\mathrm{nm}$ wide binned) light curves in the near-infrared spanning from $1.128 \mu \mathrm{m}$ to 1.634 $\mu \mathrm{m}$. In this work we aim to fill the gap between these two studies by probing the wavelength region between 738 and $1018 \mathrm{~nm}$ using the FORS2 spectrograph at the VLT.

[This chapter] is organized as follows. In Sect. 2.2, we will give details of the 
observational set up. This Sect. will be followed by a description of the data reduction in Sect. 2.3. Our investigation of instrument specific systematic noise will be presented in Sect. 2.4, and the implementation of our findings to correct the noise and the resulting obtained planet radius measurements at several different narrow wavelength channels can be found in Sect. 2.5. Our results will be discussed and set into context with existing measurements from the literature and theoretical models in Sect. 2.6. We conclude this paper with short summary and an assessment of the capabilities and limitations of current ground-based exoplanet atmosphere studies conducted with VLT/FORS2 in Sect. 2.7.

\subsection{Observations}

The hot Jupiter WASP-17b was observed on the night from the 12th to the 13th of July 2011 during transit with the FORS2 instrument (Appenzeller et al. 1998) on UT1, one of the ESO Very Large Telescopes at the Paranal Observatory. The FORS2 instrument was configured for medium resolution spectroscopy and used in multi-object spectroscopy mode. The latter was realized by using a custom made spectroscopy mask, in which slits at the position of WASP-17b and seven reference stars of similar brightness were cut. These slits were $10^{\prime \prime}$ wide and $30^{\prime \prime}$ long. The broad-slit width prevented any possible adverse effect of slit losses due to variable seeing and imperfect telescope tracking. The slit length of $30^{\prime \prime}$ permitted the measurement of the sky background for each target. The integration time was varied within the range between 20 and 30 seconds during the night to optimally adapt to changes in the observing conditions. The observations were started at 23:47 UT, $1.8 \mathrm{~h}$ before the transit ingress and ended at $6: 23 \mathrm{UT}, 0.5 \mathrm{~h}$ after transit egress. Over the whole duration of the night 373 spectra were taken for each of the reference stars and WASP-17. During the observations the rotation of the observed field on the sky was compensated by the instrument de-rotator, which ensured constant alignment of the slits on the stars. The observations were started at an air mass of 1.47. After the start of the observations the target was rising up to an air mass of 1.002, culminating while the planet WASP-17b was in transit, and sank again to a an air mass of 1.48 at the end of the observations. Due to imperfect telescope tracking, atmospheric dispersion and/or a change of the optical path due to flexures in the instrument the position of the stars within their slit changed slowly during the night. The maximal measured displacement in both directions of $\approx 2$ pixel $=0.5^{\prime \prime}$ is negligible against the large chosen slit width of $10^{\prime \prime}$ and the stars did at no point drift out of the slit.

The $600 \mathrm{z}$ grism was used to disperse the light over the wavelength range 738-1018 $\mathrm{nm}$ and the OG590 filter was used to separate the first order spectra. Due to the broad-slit width the effective resolution of the observed spectra was limited by the stellar point spread function (PSF). The conditions during the night were clear and the seeing was relatively stable at around $1.1^{\prime \prime}$. Taking this into account we derive an effective spectral resolution of $R \approx 1700$ at $800 \mathrm{~nm}$.

After the observations arc spectra of an emission lamp, exhibiting well documented emission lines, were taken to enable later wavelength calibration of the stellar spec- 
tra. These calibration spectra were taken with a slightly altered clone of the original spectroscopy mask, in which the slits were set to the smaller width of $0.1^{\prime \prime}$.

Eleven bias and 50 flat frames were obtained on the day after the observations. Following ESO standard procedure these flats were taken by using an internal lamp as the source of illumination.

\subsection{Data reduction}

The data reduction and spectral extraction of one-dimensional spectra from the two-dimensional images was realized with an IDL implementation of the 'optimal extraction algorithm' introduced and described in detail by Horne (1986). This algorithm encompasses the usual data reduction steps such as the correction of the raw CCD science frames by subtraction of the median averaged bias frame and posterior division by the median averaged flat frame as well as the subtraction of the sky background. The estimation of the sky background level is described in detail in Sec. 2.3.1. During the spectral extraction bad pixels and pixels affected by cosmic ray strike were masked. They were identified by filtering all pixels with values highly deviant $(4 \sigma)$ from an empirically derived 'expected profile'. This expected profile was calculated as the median averaged profile of the circumjacent wavelength regions in an interval of $5 \mathrm{~nm}$.

\subsubsection{Background estimation}

The sky background and its variance were estimated individually for each star and every detector column in dispersion direction by calculating the median average of all illuminated pixels with a minimum distance to the center of the spatial star profile of 20 pixels $\equiv 5^{\prime \prime}$ for each of the $30^{\prime \prime}$ long spectroscopy slits. In this calculation of the median average only values within $5 \sigma$ were considered, where $\sigma$ is the standard deviation of the background values. In case these designated background flux regions were contaminated by flux from a faint background star the corresponding pixels were excluded from the estimations. The result for the sky background flux was then subtracted from the data.

\subsubsection{Wavelength calibration and detector drift correction}

A calibration spectrum was extracted from the arc lamp frames for each star at the respective spatial detector position of this star. The corresponding wavelength solution was calculated by fitting a third-order polynomial function to the pixel positions of all identifiable lines in the calibration spectrum. The progression of the wavelength with pixel position in dispersion direction for the used set up was almost linear, but the additional orders significantly improved the fit quality. The residuals from the polynomial fit typically were of the order of $0.006 \mathrm{~nm}$ and the typical value of the linear dispersion term was $0.160 \mathrm{~nm}$ pixel $^{-1}$. 
The wavelength solution was conferred to the spectra by re-binning the spectra to a new wavelength grid with a spacing of $0.1 \mathrm{~nm}$. The re-binning was realized with the IDL routine rebinw from the PINTofALE package (Kashyap \& Drake 2000), which guarantees flux conservation. In this step we introduced two additional correction terms to the wavelength solution, which ensured that all spectra were perfectly aligned in wavelength. The first correction term was made necessary by the drift in dispersion direction within the slit that the stars exhibited during the night (as mentioned in Sect. 2.2) and which manifests as a shift in wavelength. The drift of the stars causes only very small offsets from a nearly linear wavelength solution. Therefore, it is sufficient to correct them with a linear correction term, which we determined from a cross correlation of the spectra. For this purpose all stellar spectra from each exposure were cross correlated with the corresponding stellar spectrum from the first exposure. The second correction term compensates the error from the initial centering of each star within the slit and is calculated by cross correlation of each star's first spectrum with WASP-17b first spectrum. As we only wish to correct shifts introduced by the observing set up but not those of astrophysical origin, i.e. shifts in stellar lines originating from different radial velocities of these stars, we chose windows around the telluric oxygen and water lines for this second cross correlation. This ensures that the reference star spectra are aligned with the target spectra and can accurately be used to correct variations in Earth's atmospheric transparency.

\subsubsection{Creation of light curves}

We create 14 absolute photometric transit light curves in different wavelength channels by summing the spectra of all stars over wavelength into bins of $20 \mathrm{~nm}$ width for every exposure. The choice for the bin-width of the channels was made by testing the decline of noise in the relative light curve with rising bin width. In this test noise was measured as the normalized standard deviation of the out of transit light curve interval between the frames 335-360 and compared to the theoretical progression of the noise expected from photon statistics. As can be seen in Fig. 2.1 the noise level does not further decrease significantly for larger bins after a bin width of $20 \mathrm{~nm}$.

The time stamp for every exposure was determined by using the MJD-OBS key word from the header and correct that date to the time of midexposure by adding half the duration of the exposure time. These time stamps were then converted to the Barycentric Julian date with the use of the IDL routine BARYCEN provided by Eastman et al. (2010).

In order to correct the effect of variable telluric absorption the absolute light curves of WASP-17 had to be divided by the absolute light curve of a reference star in the respective wavelength channel, yielding a set of relative light curves. For this purpose the absolute light curves of any of the reference stars could be used. Since it is desirable to maintain a high signal-to-noise ratio for the resulting relative light curve, however, generally an artificial reference star is calculated from summing the flux of all available reference stars. We found that for any chosen reference star the resulting relative light curves showed strong non-astrophysical systematic noise superimposed to the expected planet transit signal. This systematic noise appeared 


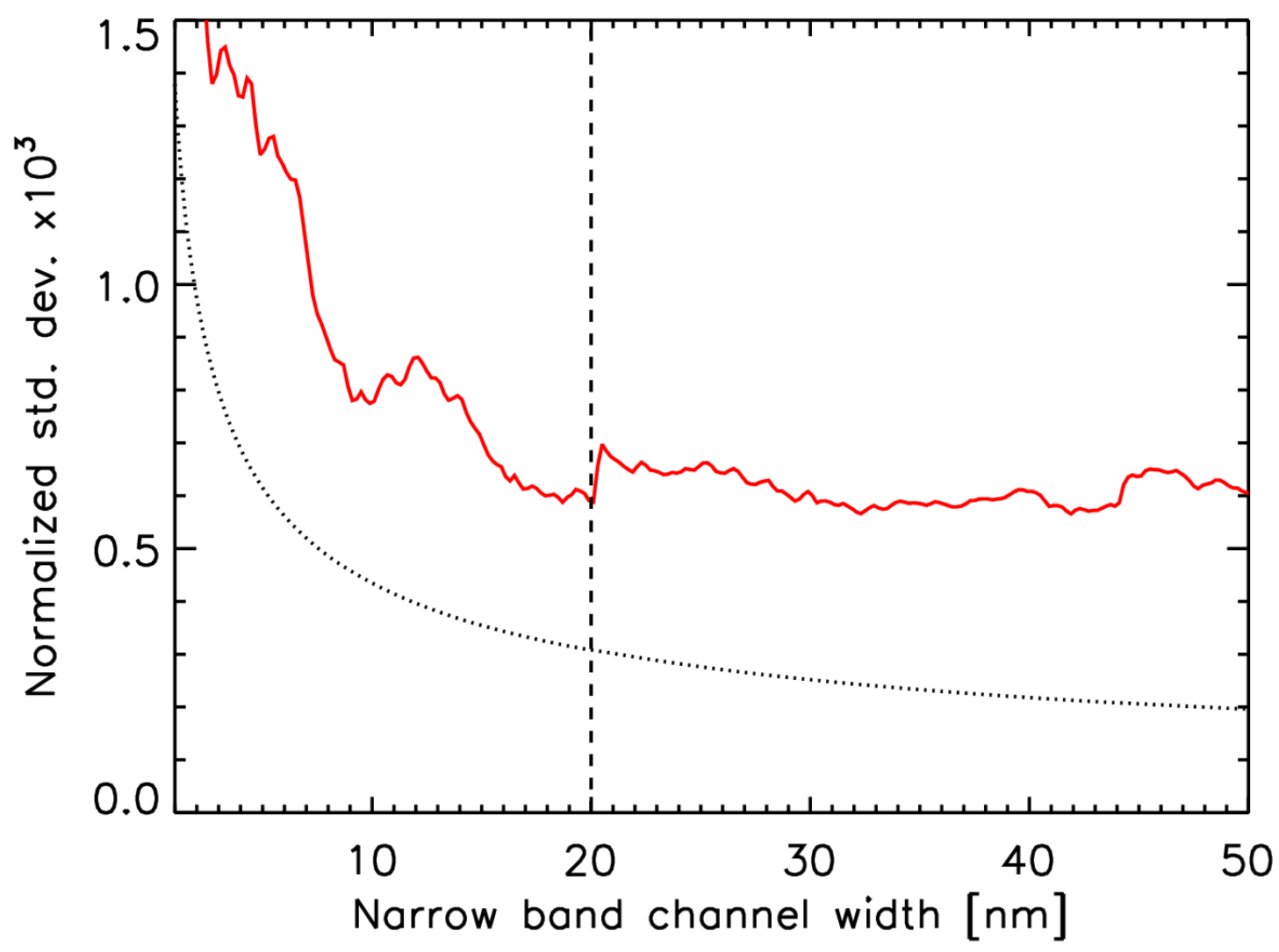

Figure 2.1: Progression of the standard deviation of a binned narrowband relative light curve with bin size (solid red line) compared to the expected theoretical progression based on photon-noise statistics (dotted black line). The vertical dashed line indicates the $20 \mathrm{~nm}$ bin-width we chose to create narrowband channel light curves.

to be of instrumental origin and was affecting the data of every star in a different manner. Consequently, the noise was manifesting in any given relative light curve as a combination of the noise affecting the target star and the noise affecting the reference star.

The contribution of the instrument noise associated with each reference star to an artificial reference star, i.e. the sum of all reference stars would vary with wavelength just as the flux contributed by each reference star is wavelength dependent. As a result the use of an artificial reference star would lead to a significantly more complicated systematic noise behavior of the relative light curve than the use of a single reference star would do. We, hence, refrained from using a summed up artificial reference star, and in the following only consider the investigation of the relative light curves constructed from division by the brightest available reference star. All other reference stars are significantly dimmer and consequently yield too noisy light curves. Figure 2.2 shows a finding chart in which WASP-17 and the used reference star are marked and the coordinates of both stars are given in Table 2.1. In Fig. 2.3 a spectrum of WASP-17 and of the used reference star are displayed. Lines indicate the $20 \mathrm{~nm}$ broad wavelength intervals over which the flux was integrated to yield 
2 Investigation of the highly irradiated atmosphere of WASP-17b in the red optical with VLT/FORS2

\begin{tabular}{|c|c|c|c|c|}
\hline Star & RA & DEC & $I[\mathrm{mag}]$ & $J[\mathrm{mag}]$ \\
\hline & $59 \mathrm{~m} \mathrm{50.947s}$ & $-28^{\circ} 03^{\prime} 42.33^{\prime \prime}$ & 10.635 & 10.509 \\
\hline Reference star & $15 \mathrm{~h} 59 \mathrm{~m} 26.392 \mathrm{~s}$ & $-28^{\circ} 01^{\prime} 17.37^{\prime \prime}$ & 10.635 & 10.205 \\
\hline
\end{tabular}

Table 2.1: Coordinates of the planet host star WASP-17 and the reference star used in this work.

the 14 narrowband light curves shown in Fig. 2.3.

Fig. 2.4 shows an example of a resulting relative light curve. The non-astrophysical noise in the light curve can be roughly described by a relatively smooth inverted s-shaped slope interrupted by two sharp jumps/drops in the relative flux level.

We find that even with the use of only one reference star that the systematic noise is not identical for every of the $20 \mathrm{~nm}$-binned light curves, i.e. is wavelength dependent. Consequently, the systematics cannot be easily removed by taking the ratio of the individual curves but have instead to be better understood and then modeled with a more advanced approach. In the following section, Sect. 2.4, we describe our studies of the origin of these systematics. In Sect. 2.5 we use the gained insight obtained from these studies and implement a model to correct the noise structures from the data.

\subsection{The LADC - The origin of the systematic noise in FORS2 light curves}

We found that flux jumps seen in the light curve coincide with times at which the Longitudinal Atmospheric Dispersion Corrector (LADC) was reset in order to optimally adapt to the actual observing conditions. The LADC (Avila et al. 1997) is situated in the M3 tower of the UT1 telescope and consequently located in the optical path of the instrument. It consists of two prisms whose distance can be regulated in order to optimally compensate the dispersion effect induced by the actual atmospheric conditions. In normal operations mode the LADC prism distance is adjusted at the beginning of every Observing Block and will be re-adjusted should this Observing Block be stopped and re-started. The value of the LADC prism distance for every frame during the observing night is shown in Fig. 2.5.

While the CCD detector, the spectroscopy mask, the grism and the filter are rotated by the instrument de-rotator to compensate the Earth's rotation, the LADC angle is fixed and the device moves only in altitudinal and azimuthal direction with the telescope. Consequently, the path along which the starlight passes though the two LADC prisms before reaching the detector is not fixed over the whole duration of the observing night. In 2010 Moehler et al. (2010) discovered that the LADC prisms exhibit a nonhomogenous transmittance over their surface. The problem was brought to light while investigating a flat field feature which appeared to be rotating with the de-rotator angle and, thus, could not be caused by the filter or detector but had instead to be caused by a part of the instrument that is located in front of the de-rotator. This study and its implications for broadband photometry 


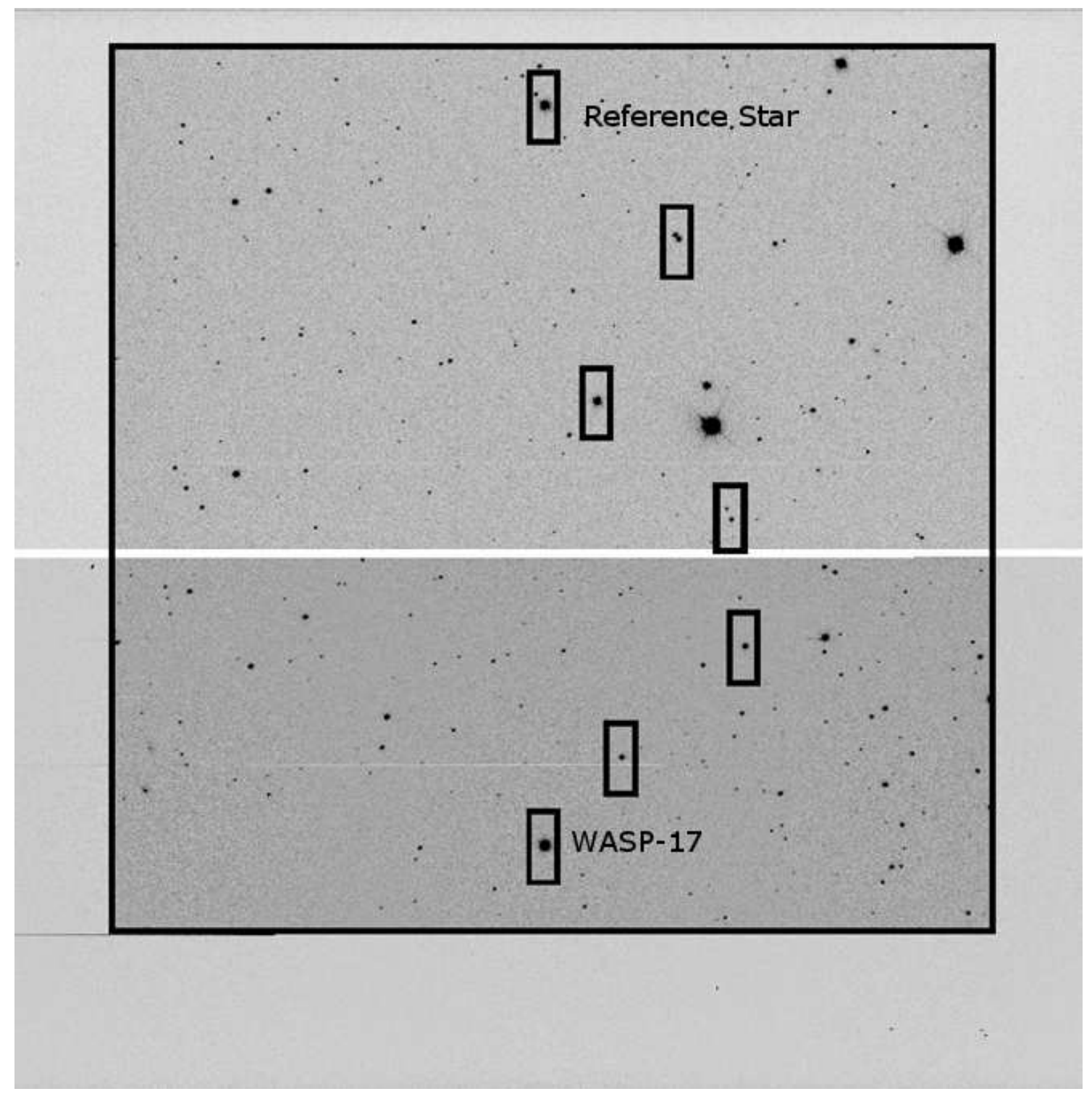

Figure 2.2: Finding chart in which WASP-17 and the used reference star are marked.

conducted with FORS2 was recently followed up by Coccato et al. (2014).

Adopting the approach of Moehler et al. (2010) we downloaded all sky flats obtained in the $z$ _GUNN filter in the year 2011 from the ESO archive (330 frames). We corrected these flats by a median flat and de-rotated the residuals by the value of the instrument de-rotator angle under which they had been obtained. Then we averaged these rotated residuals and, thus, created an image of the rotation dependent inhomogeneous transmittance pattern of the FORS2 instrument (in the following abbreviated as RP for 'rotation pattern'). For each frame taken during the observing night of June 11th 2011, we determined the position at which the star light of each star passed the LADC prisms. The resulting traces of WASP17 and the reference star on the RP are shown in Fig. 2.6. We extracted the corresponding transmittance curves for both stars from the RP and divided them to determine the expected effect of the LADC transmittance on the relative light 


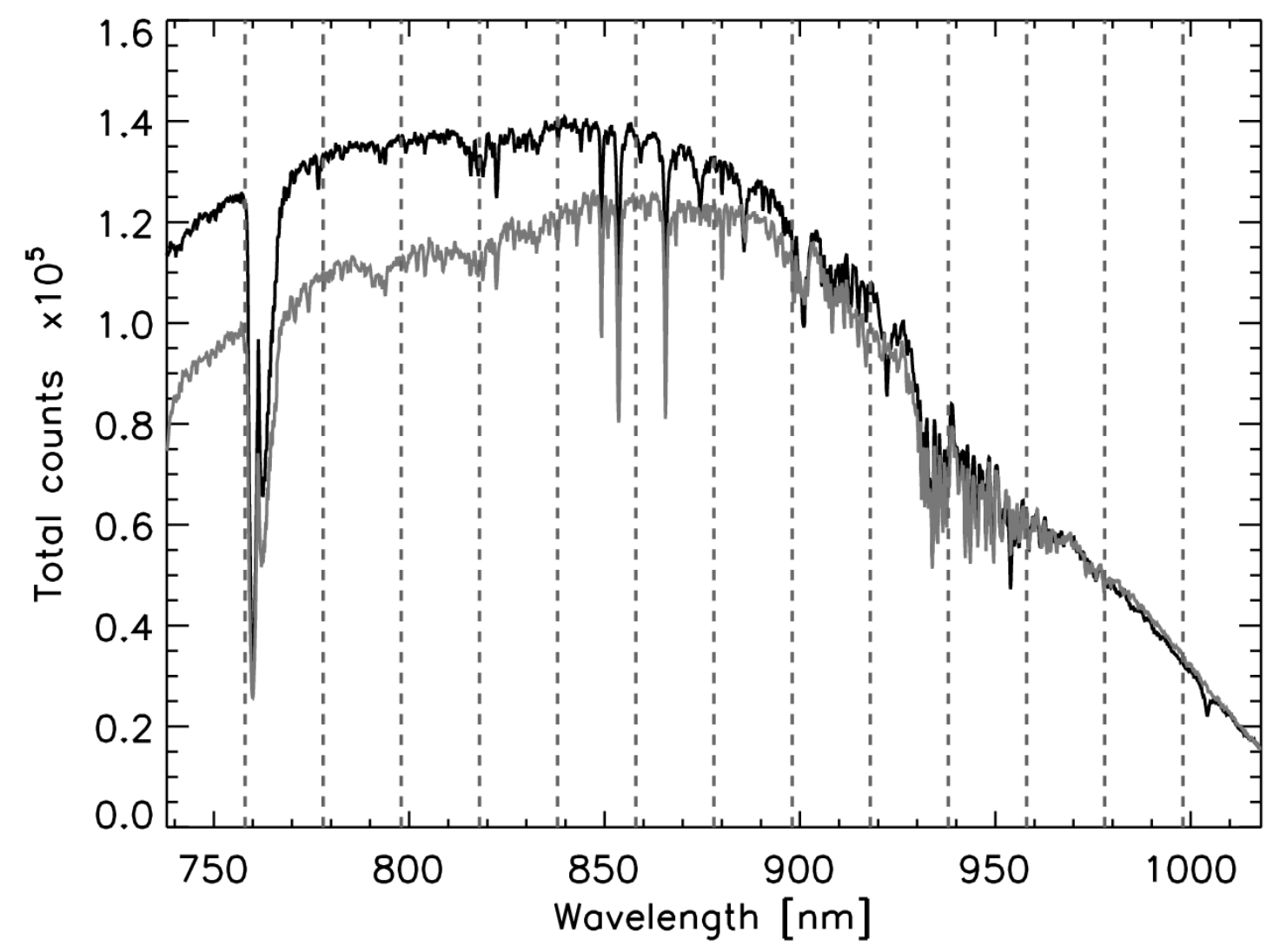

Figure 2.3: Spectra of WASP-17 (black) and the used reference star (gray). Lines indicate the limits of the $20 \mathrm{~nm}$ wide wavelength bins in which the flux for each observed spectrum was summed to create fourteen narrowband light curves.

curve. The resulting transmittance curves are displayed in Fig. 2.7 and are in good general agreement with the s-shaped systematic noise found in our actual data. The agreement between the extracted transmittance curve and our data is, however, not sufficient for a satisfying correction of the noise. The amplitude of the transmittance variation in the extracted curve is smaller than in our data. Even if we allow the signal to scale before we divide our data by it and allow the jumps to be compensated for by three independent offsets, the correction is still poor for the region in which the paralactic angle changed fast during the observation (frames 150-250, see Fig. 2.5 and 2.7), leaving a remaining strong upwards distortion in the transit bottom of the corrected curve. The shortcoming of the RP as a correction of our light curve is not surprising since the RP was constructed from a limited number of flats that were taken in a broadband filter (not in spectroscopy) and at randomly distributed LADC prism distances. The latter clearly exhibiting an influence on the overall level of transmittance. A sufficient correction for the systematics from flat field data, thus, would require a significantly larger number of spectroscopic sky flats taken at every rotation angle, and every (used) LADC prism distance. A less intricate solution for a correction of a specific relative transit light curve could have been a repetition the observation during a following or preceding night where no 


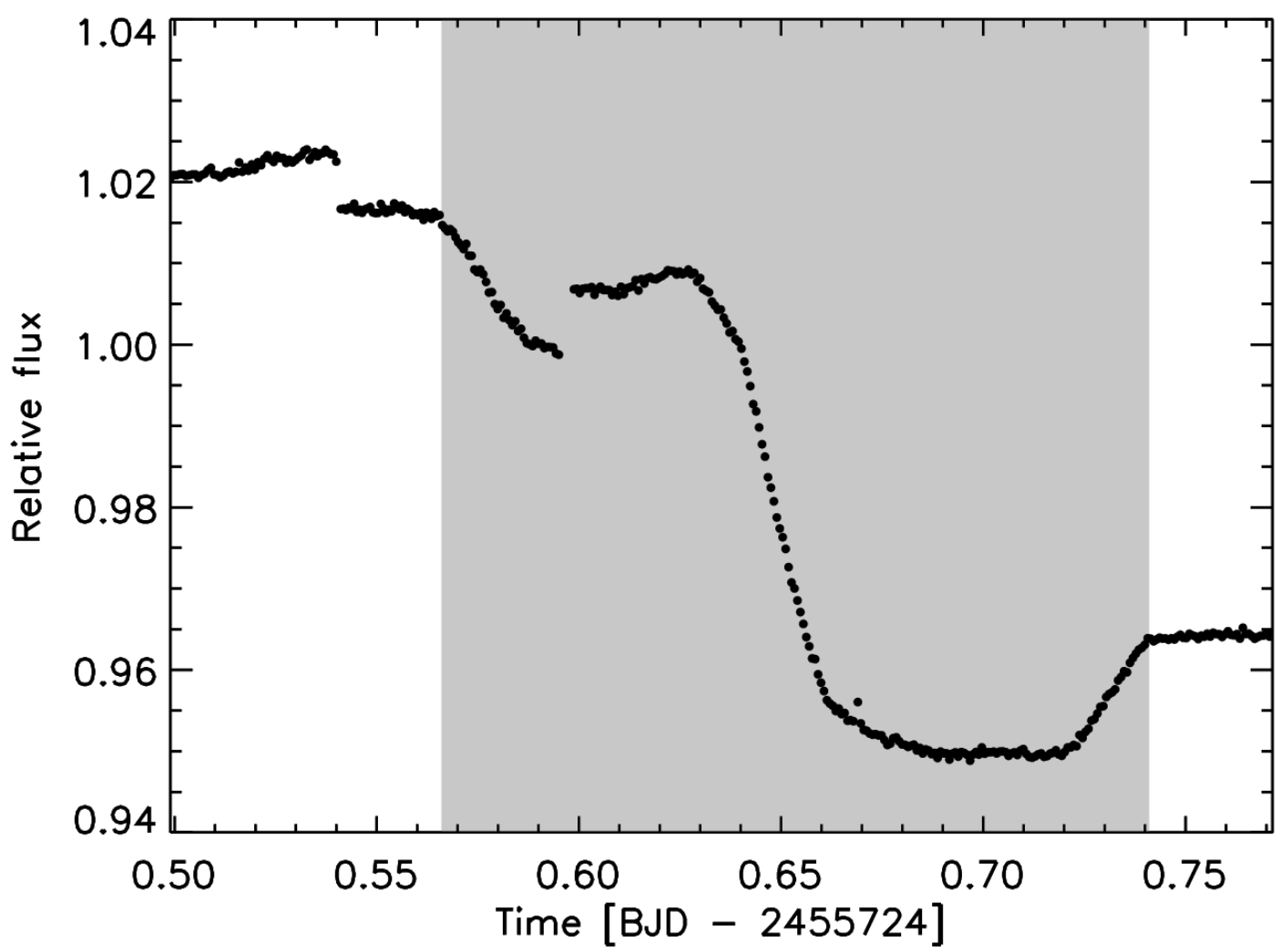

Figure 2.4: Example relative light curve that shows the transit signal superimposed by an inverse s-shaped non-astrophysical systematic noise and jumps. The time in which the transit occurs is marked in gray.

transit event occurred using the same instrument set up and observing the objects under the same paralactic angles while keeping the LADC prism distance fixed. If no further, yet unknown, systematics affect the light curves then such a division of the transit light curve by the next/previous day 'out-of-transit' light curve should have yielded an accurate correction. As the LADC prisms were exchanged in November 2014 (see. Sect. 2.4.2), such observations cannot be conducted anymore as follow-up for archive transit data obtained prior to this date.

\subsubsection{Occurrence of instrument systematics in previous spectroscopic FORS2 observations}

If our assumptions about the source of the instrument systematics are correct, they should have also affected data previously taken with the instrument. If so, one might ask how it was possible to obtain the successful observations of GJ 1214b made by Bean et al. in 2010 and 2011. This can be explained when we consider that the transit of GJ $1214 \mathrm{~b}$ is rather short $(52.7 \mathrm{~min})$ compared to the one of WASP-17b (262.8 min) and that due to GJ 1214's position in the sky over Paranal the paralactic angle of the observation only changes very slowly. If the timing of the transit is convenient and does not coincide with the meridian passage of the 
2 Investigation of the highly irradiated atmosphere of WASP-17b in the red optical with VLT/FORS2

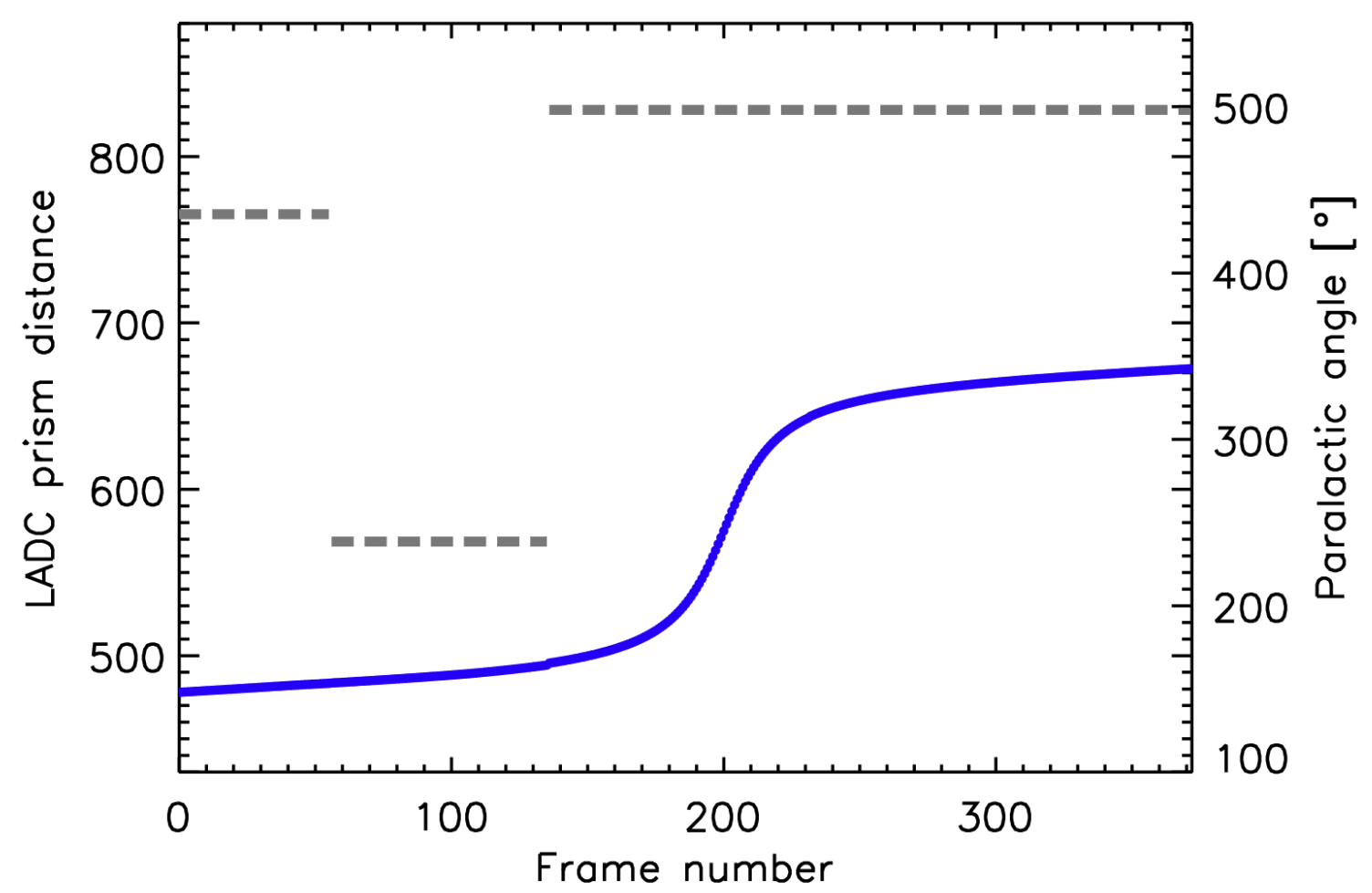

Figure 2.5: The progression of the LADC prism distance (dotted line) and the paralactic angle (solid line) during the observations of WASP-17.

target, where the paralactic angle would change faster, then only a small region of the LADC prism inhomogeneity pattern is sampled leading to a very small and less complex instrument systematic signal. As an example we show in the top panel of Fig. 2.8 the almost linear change in paralactic angle during the observations of GJ 1214b taken of April 29th 2010, (one of the two data sets used in Bean et al. (2010)). The LADC prism distance was not yet included as an auxiliary parameter in the header in 2010 but the observations were not stopped at any point and, thus, it is safe to assume that the prism distance was constant over the whole duration these observations. If we investigate the stellar traces of GJ 1214 and the five used reference stars (see Fig. 2.9) and derive an estimate for the expected signal affecting the light curves we yield a signal which can be described by a simple linear function in time (see Fig. 2.8, bottom panel). In this rough estimate we neglected that the five reference stars would all contribute to the summed up reference star with a slightly different weight due to their brightness differences. Bean et al. (2010) found their light curves to be affected by a time dependent curvature which they stated to be most likely caused by color differences between the target star and the reference stars. They corrected the curvature by de-trending their light curves with second order polynomial function in time, effectively modeling the instrument systematics together with the possible color effect. 


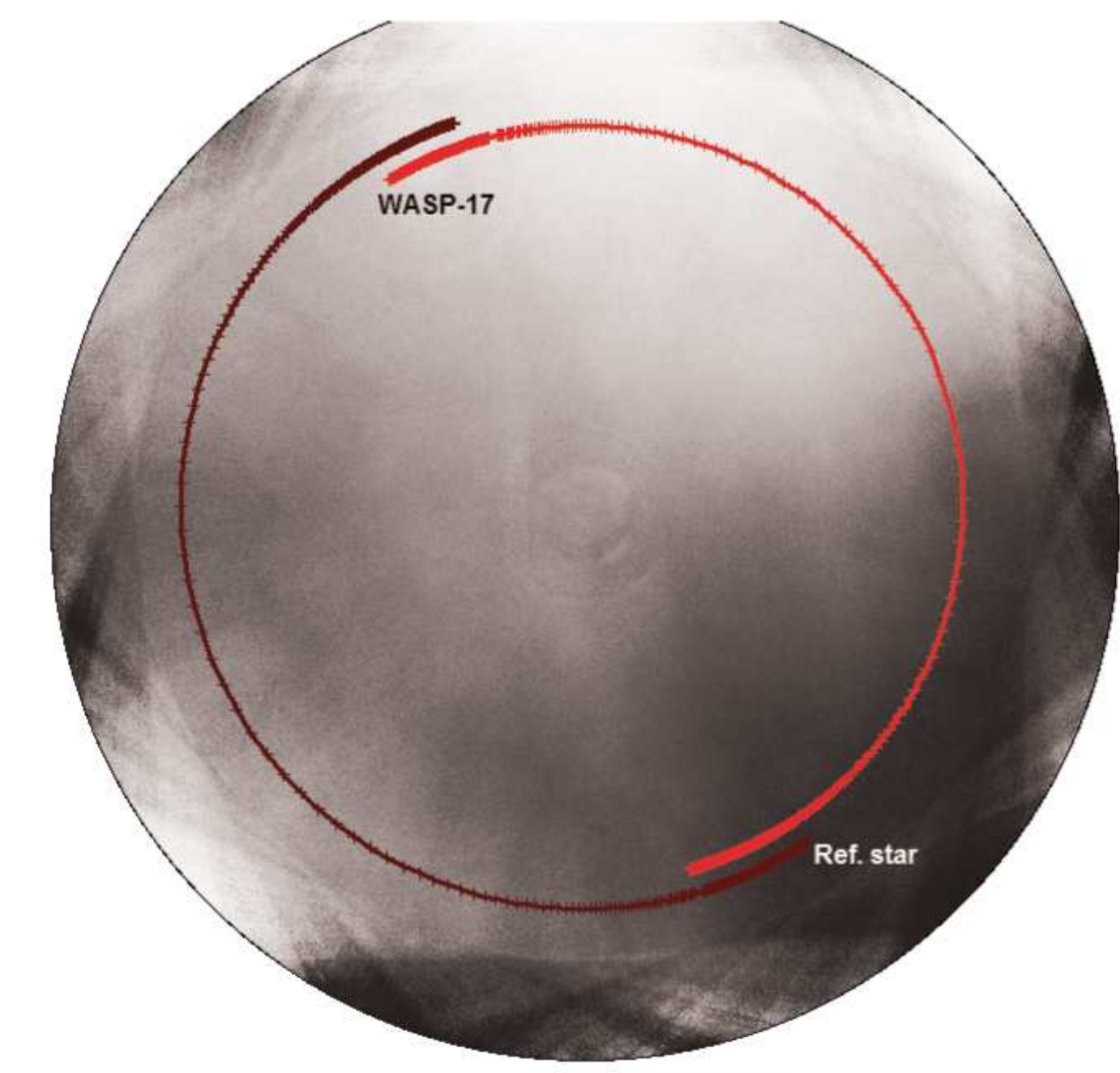

Figure 2.6: Image of the rotation dependent inhomogeneous transmittance pattern of the FORS2 instrument (short RP). Indicated are the traces of WASP-17 (inner half circle trace, progressing from the top of the image clockwise to the bottom) and the reference star (outer half circle trace, progressing from the bottom of the image clockwise to the top) on this pattern during the observing night. 


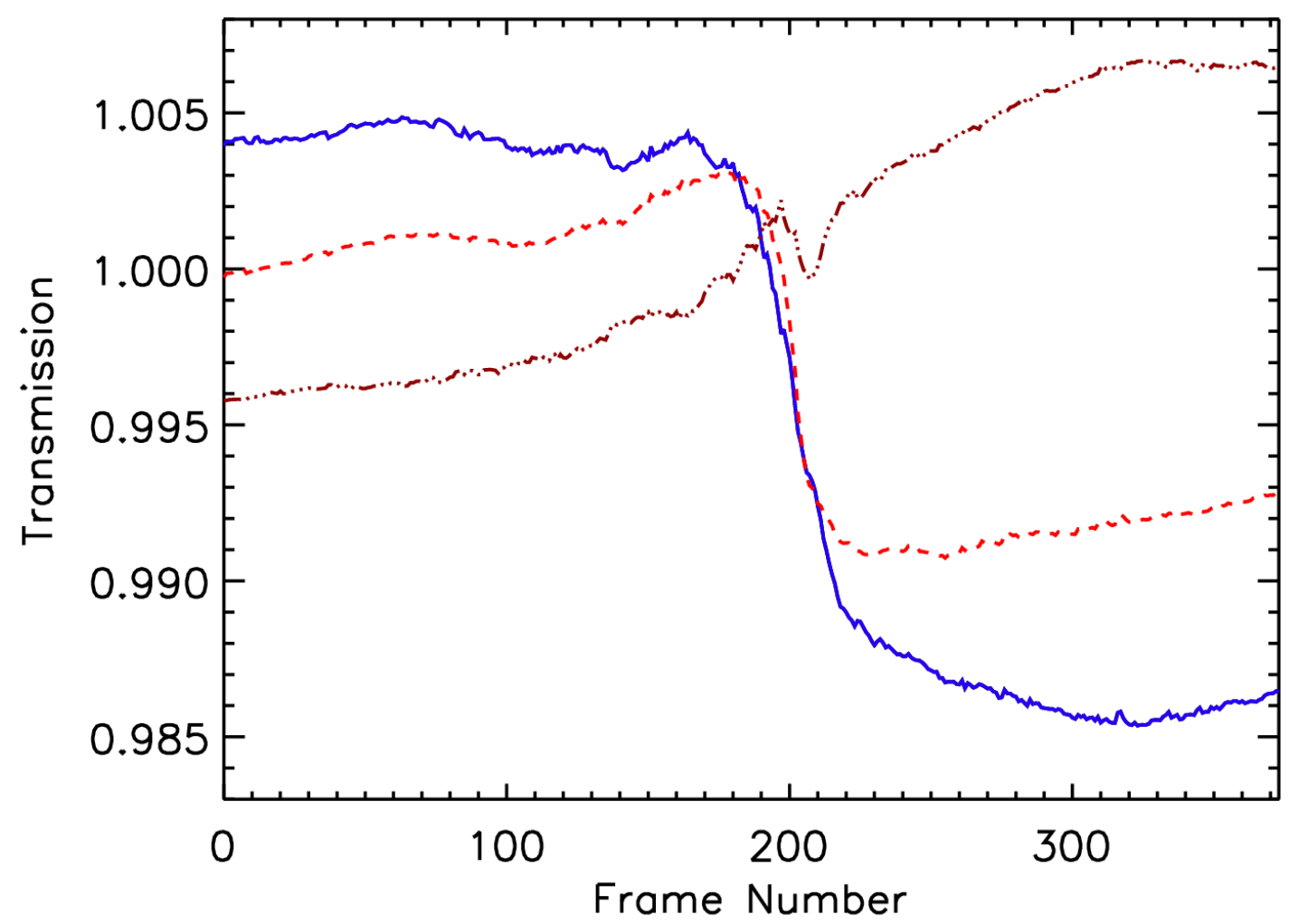

Figure 2.7: The extracted transmittance curves of WASP-17 (dashed and dotted line) and the reference star (dashed line) and their ratio, which corresponds to the expected instrument noise in the relative light curve (solid line).

\subsubsection{Replacement of the FORS2 LADC prisms}

In response to the lack of FORS2 based transit transmission spectroscopy publications after the initial successes of Bean et al. (2010, 2011), despite the abundance of granted observation time, the European Southern Observatory (ESO) finally decided to replace the compromised FORS2 LADC prisms with their identical counterpart of the shelved FORS1 in 2014. Boffin et al. (2015) report that the variation in the LADC's transmittance is suspected to be related to the $\mathrm{MgF}_{2}$ antireflective coating of the prisms, which started degrading after an effort was made to clean them in 1999, confirming the initial suspicion of a relation with the prism coating by Moehler et al. (2010). The antireflective coating of the FORS1 LADC was fully removed previous to its installation at UT1, which occurred during maintenance in November 2014. The subsequent testing of FORS2's performance with the renewed prism by observing WASP-19b in transit yielded a significant reduction in the observed systematics when compared to data of the same planet taken in April 2012 (Boffin et al. 2015, Sedaghati et al. 2015). It is worthwhile mentioning, however, that WASP-19b similar to GJ 1214b, has a very short transit duration and can be observed under very little variation of the paralactic angle. Therefore, under favorable conditions only very subtle systematics could be present in the data, while at other less favorable conditions systematics might be more obvious. Observing this planet is, thus, a first step in confirming an improvement of the FORS2 situation, but should be 


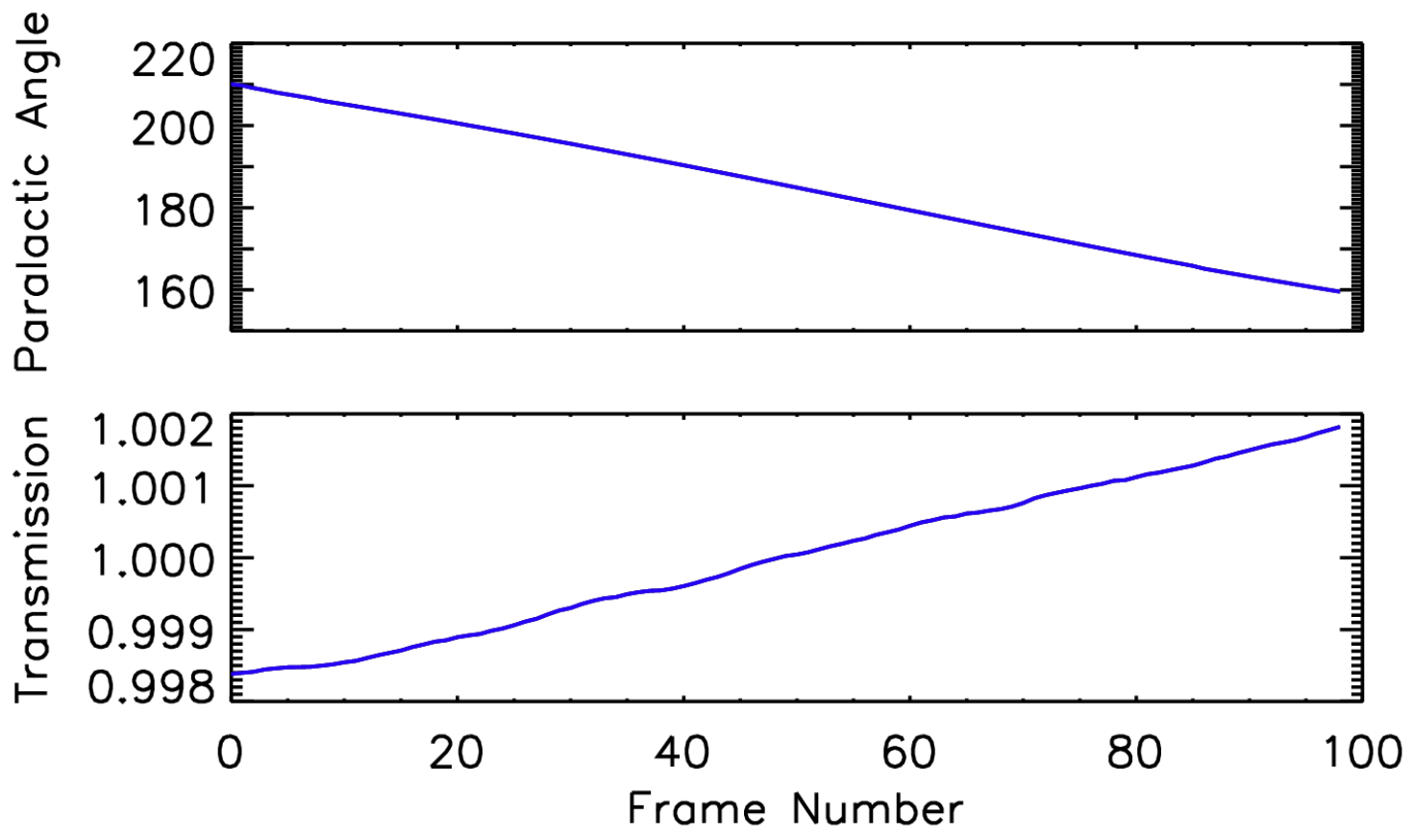

Figure 2.8: Top panel: the progression of the paralactic angle (solid line) during the FORS2 observations of GJ 1214 on April 29th 2010. Bottom panel: the ratio of the extracted transmittance curve for GJ 1214 and the sum of the transmittance curves of all reference stars, which provides a rough estimate of the expected instrument noise in the relative light curve.

followed up by using an exoplanet with longer transit duration that is observed over a period with large and fast changes of paralactic angle.

\subsection{Analysis and results}

The instrument induced systematic noise dominates the data and has to be corrected to make an investigation of the planet parameters possible. The transmittance curve extracted from the de-rotated sky flats is not sufficient for a correction of the noise. Therefore, we opted to handle these non-astrophysical systematic trends with a parameterized model based on our understanding of their origin which will be fitted to the data together with an analytical transit model describing the astrophysical information in the light curve. We tested this modeling approach on a 'white' light curve of our data, which we created by combining all the 14 narrowband light curves after each of these 14 curves was normalized to the out of transit flux level measured after the egress. This approach is different from the usual construction of a 'white' light curve, in which all measured flux is summed, without prior normalization. Our approach reduces possible effects of differential atmospheric absorption that could cause air mass dependent trends in the relative light curve due to color differences between the target and reference star.

The transit model was realized using the analytical model introduced by Mandel \& Agol (2002) as implemented in code by Eastman et al. (2013). The planet system 


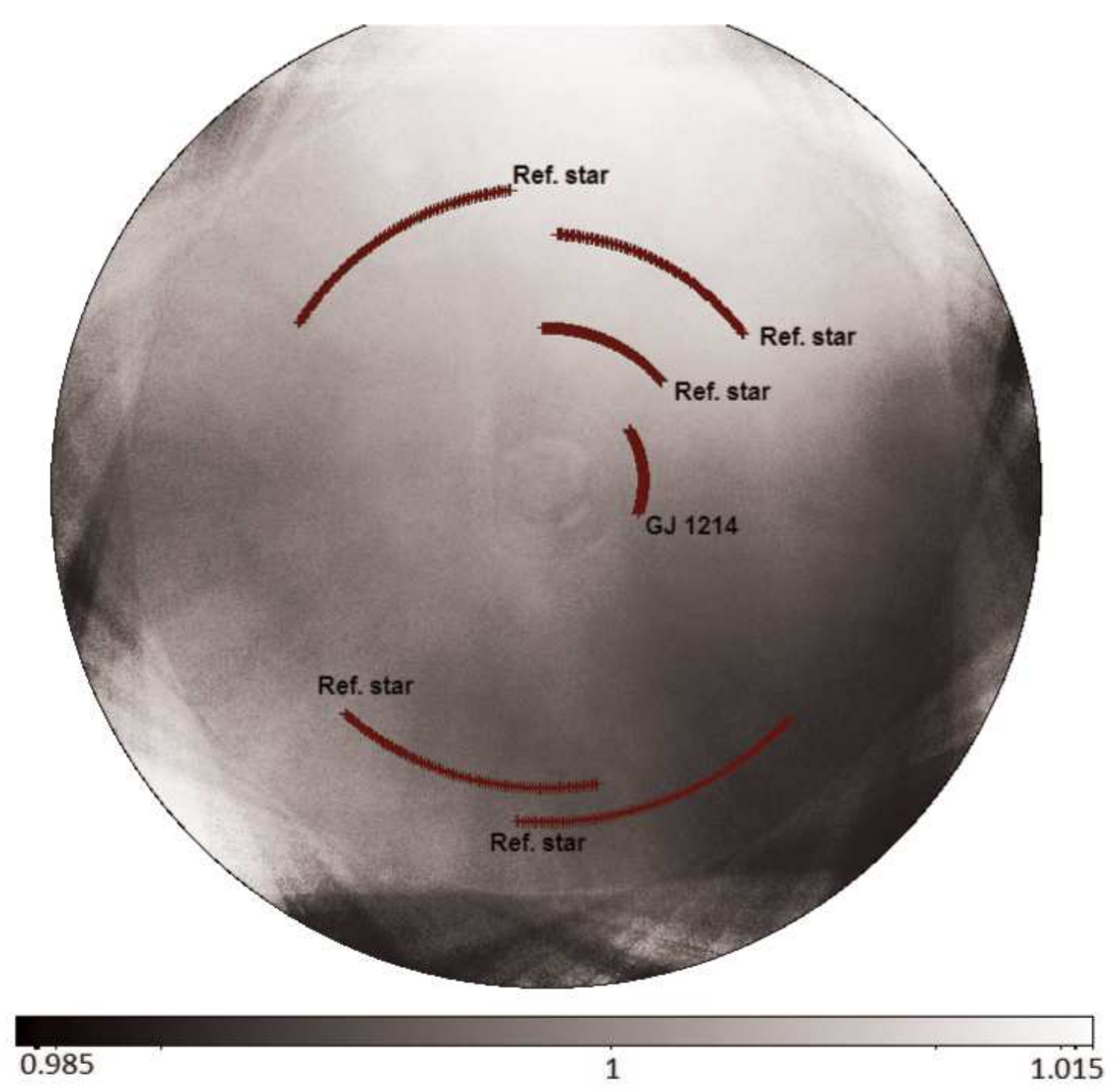

Figure 2.9: The traces of GJ 1214b and all five used reference stars from the April 29th 2010 FORS2 observations published in Bean et al. (2010) are shown on top of the rotation dependent inhomogeneous transmittance pattern of the FORS2 instrument (short RP). The position of each star at the beginning of the night is indicated by the position of the trace label. The star positions then progressed counterclockwise during the night. 
parameters needed in this model are the radius ratio between the planet and the star $R_{\mathrm{p}} / R_{\star}$, the semimajor axis of the planet's orbit in units of the stellar radius $a_{\mathrm{p}} / R_{\star}$, the inclination of the planet orbit $i$, a reference time for the midtransit $T_{\mathrm{C}}$ and two coefficients $u_{1}, u_{2}$ describing the stellar limb darkening with a quadratic limb darkening law. Also needed is the period of the planet's orbit $P$, with we adopt and fix to the value given by Southworth et al. (2012) $P=3.7354331$ and the eccentricity of the orbit $e$, which we kept fixed at $e=0$.

\subsubsection{Limb darkening}

The limb darkening parameters can be fitted in case of very high quality light curves. Due to the strong systematic noise in our case, leaving the limb darkening parameters free resulted in them assuming un-physical values. We, therefore, decided to keep both parameters fixed to theoretical values for the 'white' light curve fit. For this purpose we derive the values of both quadratic stellar limb darkening coefficients for all 14 narrowband wavelength channels and for the 'white' light curve using a spherically symmetric stellar model atmosphere calculated with the PHOENIX code (Husser et al. 2013). We interpolate the grid of available model atmospheres to yield the limb darkening data for a star with the properties of the host star WASP-17 $\left(T_{\text {eff }}=6550 \mathrm{~K}\right.$, Metallicity $\left.[\mathrm{Fe} / \mathrm{H}]=-0.25, \log g(\operatorname{cgs})=4.149\right)$, then bin the model star's angle dependent intensity curves over the same wavelength intervals as the WASP-17 light curve data and fit the results for each channel (and the 'combined channel white light') with a quadratic limb darkening law.

\subsubsection{Modeling the instrument noise in the 'white light' curve}

The position of the stars on the LADC prisms change with the paralactic angle $\theta_{\mathrm{PA}}$ (or instrument de-rotator angle, which is identical with the former except for a constant offset), and in addition to this the overall transmittance changes as a function of the LADC prism distance $\delta_{\mathrm{LADC}}$. The progression of both the LADC prism distance and the paralactic angle during the observing night is shown in Fig. 2.5. We used these two auxiliary parameters to model the systematic noise as a polynomial function and experimented with different degrees of complexity of the model for the instrument noise $\mathcal{C}$, by allowing the order of the polynomial terms to grow.

In these experiments we used the IDL implementation of the Levenberg-Markward algorithm mpfit (Markwardt 2009) to determine the minimal $\chi^{2}$ when modeling the 'white' light curve with a model $\mathcal{M}$ of the form:

$$
\mathcal{M}=\mathcal{C} \cdot \mathcal{T}
$$

Where $\mathcal{T}$ is the Mandel \& Agol transit model.

While higher complexity of the model was accompanied by a lower $\chi^{2}$, a trade of between the number of free parameters and $\chi^{2}$ minimization is desired to avoid over fitting. One method to decide whether the use of an additional free parameter 

optical with VLT/FORS2

\begin{tabular}{cc}
\hline \hline Model & $\Delta B I C$ \\
\hline ladc1 paran1 & 1145.3 \\
ladc1 paran2 & 902.0 \\
ladc1 paran3 & 25.3 \\
ladc1 paran4 & 28.4 \\
ladc1 paran5 & 28.6 \\
ladc1 paran6 & 34.1 \\
ladc2 paran1 & 905.3 \\
ladc2 paran2 & 692.2 \\
ladc2 paran3 & 0.0 \\
ladc2 paran4 & 4.5 \\
ladc2 paran5 & 10.4 \\
ladc2 paran6 & 14.1 \\
\hline
\end{tabular}

Table 2.2: Model comparison. Different models and the respective $\triangle B I C=B I C-$ $B I C_{\text {min }}$ values.

is justified is the minimization of the Bayesian information criterion $B I C=\chi^{2}+$ $k \cdot \ln (N)$, where $k$ is the number of free parameters and $N$ is the number of data points. We compared the $B I C$ of all tested models and found that using a secondorder polynomial of the LADC prism distance and a third-order polynomial function of the de-rotator angle/paralactic angle yields the lowest $B I C$. The $B I C$ results for all models can be found in Table 2.2, where the models are identified by a short code, which is to be read as follows: 'ladc' is the key word for the LADC prism distance and 'paran' the paralactic angle. The number following each of these key words indicates the highest polynomial order that was allowed to be free in the model fit. The best model for the instrument systematics $\mathcal{C}^{*}$, which we chose to adopt in our final fitting routine is, thus, indicated by the key word 'ladc2 paran3' and of the form:

$$
\mathcal{C}^{*}=n_{0}+l_{1} \delta_{\mathrm{LADC}}+l_{2} \delta_{\mathrm{LADC}}^{2}+p_{1} \theta_{\mathrm{PA}}^{1}+p_{2} \theta_{\mathrm{PA}}^{2}+p_{3} \theta_{\mathrm{PA}}^{3}
$$

With $n_{0}$ the normalization, $l_{1}, l_{2}$ the free parameters regulating influence of the LADC prism distance $\left(\delta_{\mathrm{LADC}}\right)$ and $p_{1}, p_{2}, p_{3}$ the free parameters regulating influence paralactic angle $\left(\theta_{\mathrm{PA}}\right)$ in the instrument systematics model. (It might be helpful to note here that the number of free parameters for the LADC prism distance including the normalization is three, which, as we were operating under only three different LADC prism distances during the observations, is giving the model the same freedom as using three different normalizations, one for each of the LADC distances, would.) 


\subsubsection{Errors}

We use a Markov Chain Monte Carlo (MCMC) method with an affine-invariant ensemble sampler as implemented in the Python package emcee by Foreman-Mackey et al. (2013) to probe and, thus, determine the shape of the probability distribution of the model parameters. The general outlay of the MCMC procedure was very similar to what is described in (Berta et al. 2012). The procedure aims to maximize the logarithmic Likelihood function $\mathcal{L}$ given in Eq. 2.3 and we were using solely noninformative priors for all parameters.

$$
\ln \mathcal{L}=-N \ln \left(\sigma_{\mathrm{wn}}^{2} \sigma_{\text {err }}^{2}\right)-\frac{\chi^{2}}{2 \sigma_{\mathrm{wn}}^{2} \sigma_{\mathrm{err}}^{2}}
$$

In Eq. $2.3 N$ is the number of data points, $\sigma_{\mathrm{wn}}$ is a free parameter re-scaling the amplitude of the white noise and $\chi^{2}$ is given by sum of the squared residuals. The input error bars of each point $\sigma_{\text {err }}^{2}$ are in this case the same, and given by the normalized standard deviation of the points outside of the transit. The emcee package uses an ensemble of walkers that start with slightly different parameter populations as seeds for the chains. The results can be considered robust if all the chains converge to the same probability distribution. We used 300 individual chains with 10000 iterations each. We identify the burn-in phase, in which the chains are not yet fully converged, to be over after 500 steps and consequently omit these first 500 points for each chain. For every chain we identify the parameter that exhibits the largest autocorrelation $N_{\max }$ and then only accepted every $N_{\max }$ step of that particular chain. The average maximal autocorrelation length was 92 which resulted in a total of 30059 accepted sample points for the merged distribution of accepted chain steps. The correlation plots of the posterior distributions for all parameters are shown in Fig. 2.11.

The $1 \sigma$ error bars for each parameter are then calculated by taking the percentiles of the distribution at $50-(68.27 / 2$. $)=15.865 \%$ and $50+(68.27 / 2$. $)=84.135 \%$, i.e. determining the values in between which $68.27 \%$ of all sampled points lie.

\subsubsection{Red noise estimation}

We experimented with and then abandoned the wavelet approach suggested by Carter \& Winn (2009), which is designed to determine the influence of red noise on the posterior parameter distribution. It is intended for red noise with a power spectral density varying as frequency ${ }^{-\gamma}$. In this approach the Likelihood depends on 'red noise' and a 'white noise amplitude parameter' and the spectral index $\gamma$. We found that our result for the transit depth was overly sensitive to the exact choice of these parameters, which could not be determined from the data independently with sufficient confidence. Therefore, we deemed the wavelet approach to red noise consideration not suitable for our data and opted to instead use the method suggested by Winn et al. in 2008. In this approach the theoretical progression of the standard deviation of a light curve that is only affected by Gaussian (i.e. white) noise, when binned in time (see Eq. 2.4) is compared to the actual progression of the standard deviation in the binned light curve residuals. The standard deviation 
2 Investigation of the highly irradiated atmosphere of WASP-17b in the red optical with VLT/FORS2
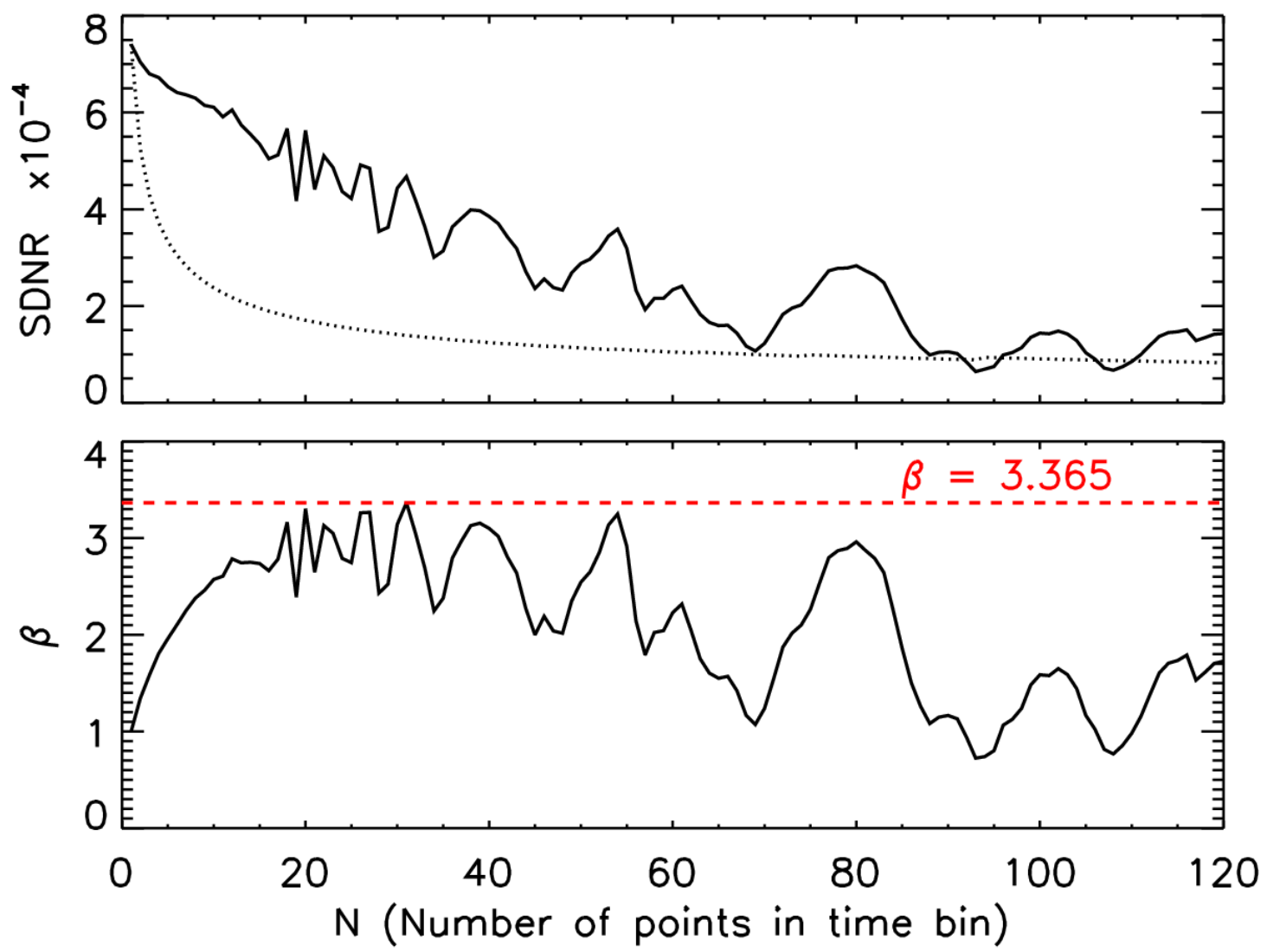

Figure 2.10: Top panel: theoretical progression of the standard deviation of a light curve purely affected by white noise (dotted line) compared to the actual progression of the standard deviation in the binned 'white' light curve residuals (solid line). Bottom panel: division of the two curves from the top panel (i.e. actual progression by the theoretical curve, solid line). We derive the 'red noise factor' $\beta$ as the maximal value of this ratio.

of a light curve affected with purely Gaussian white noise of the level of $\sigma_{1}$ should show the following behavior, if binned into $M$ bins, each containing $N_{b}$ points:

$$
\sigma_{N}^{\text {theory }}=\frac{\sigma_{1}}{\sqrt{N_{b}}} \sqrt{\frac{M}{M-1}}
$$

Any deviation between these two can be attributed to red noise. The average difference between the two curves can then be understood as a 'red noise factor' $\beta=\sigma_{N}^{\text {actual }} / \sigma_{N}^{\text {theory }}$ and be used to inflate the MCMC chain derived error bars of all parameters.

The progression of the two curves and the ratio between them is shown in Fig. 2.10. We choose to use the maximal value of beta $\beta_{\max }=3.365$ which is reached at a bin number of $N_{b}=31$. 


\subsection{5 'White light' curve results}

Figure 2.12 shows the light curve with the best-fit model for the instrument systematics. Figure 2.13 shows the light curve, after division by the instrument systematics model plotted together with the best-fit model for the transit shape. It can be seen that the residuals are still affected by red noise, which prompted us to inflate the error bars of our best-fit parameters by the red noise factor $\beta$ (see Sect. 2.5.4). The results of the optimization and the confidence intervals are given in Table 2.3 together with the results of the works by Southworth et al. (2012), Bento et al. (2014), Anderson et al. (2010) and Mandell et al. (2013), who observed in partly overlapping and neighboring wavelength regions. Our best-fit results are in general good agreement with the literature values. The radius ratio (and consequently the transit depth) is larger than the values derived for most other studies including the one in the Cousins $I$ band pass, which covers a wavelength region which is almost fully included in the wavelength interval of our measurement. Our value is consistent with the Sloan r' band measurement by Bento et al. (2014). Which itself is inconsistent with the work by Southworth et al. (2012) who observed in the very similar band pass Bessel $R$. It can be seen that the literature values for the radius ratio $R_{\mathrm{p}} / R_{\star}$ of WASP-17b are highly variable (see Fig. 2.14). As an alternative to looking at the radius ratio we will in the following work with the 'transit depth' $\delta=\left(R_{\mathrm{p}} / R_{\star}\right)^{2}$ which would be equal to the fraction of flux loss due to the stars occultation by the planet under absence of stellar limb darkening. For the study by Bento et al. (2014) we calculated the transit depth from the $R_{\star} / a_{\mathrm{p}}$ and $R_{\mathrm{p}} / a_{\mathrm{p}}$ values given in their paper and used Gaussian error propagation to derive the uncertainty intervals.

While a color dependent difference in transit depth due to the expanded atmosphere is expected, this is unlikely to explain a discrepancy as large as the one found between the $R$-band values of Southworth et al. (2012) and Bento et al. (2014) that were obtained in almost completely overlapping wavelength regions. We tested if such discrepancies could instead be caused by co-occurring differences in the remaining system parameters like the semimajor axis and the orbital inclination, which also have an influence on the shape of the transit light curve. As the data of Southworth et al. (2012) and Anderson et al. (2010) are available online, we were able to test this theory by fitting their light curves with a Mandel \& Agol transit model where the orbital parameters were fixed on the orbital parameters found by Bento et al. (2014). The result of this test was that the influence of the orbital parameters is small and the resulting transit depth were well within the error bars of the original studies, where the orbital parameters were allowed to vary. (While this result shows us that the influence of the orbital parameters on the transit depth can be neglected and results from different works can be compared with each other to derive a global picture of the atmospheric transmission over a larger wavelength range, it also points out that there are unresolved discrepancies between different studies on this planet.) The discrepancy between our result and the value from (Anderson et al. 2010) might be due to the fact that all our tested instrument systematics models are limited in the way that they approximate the effect as a smooth function. The low amplitude variations, which are clearly part of the actual signal, might be misinterpreted as a 


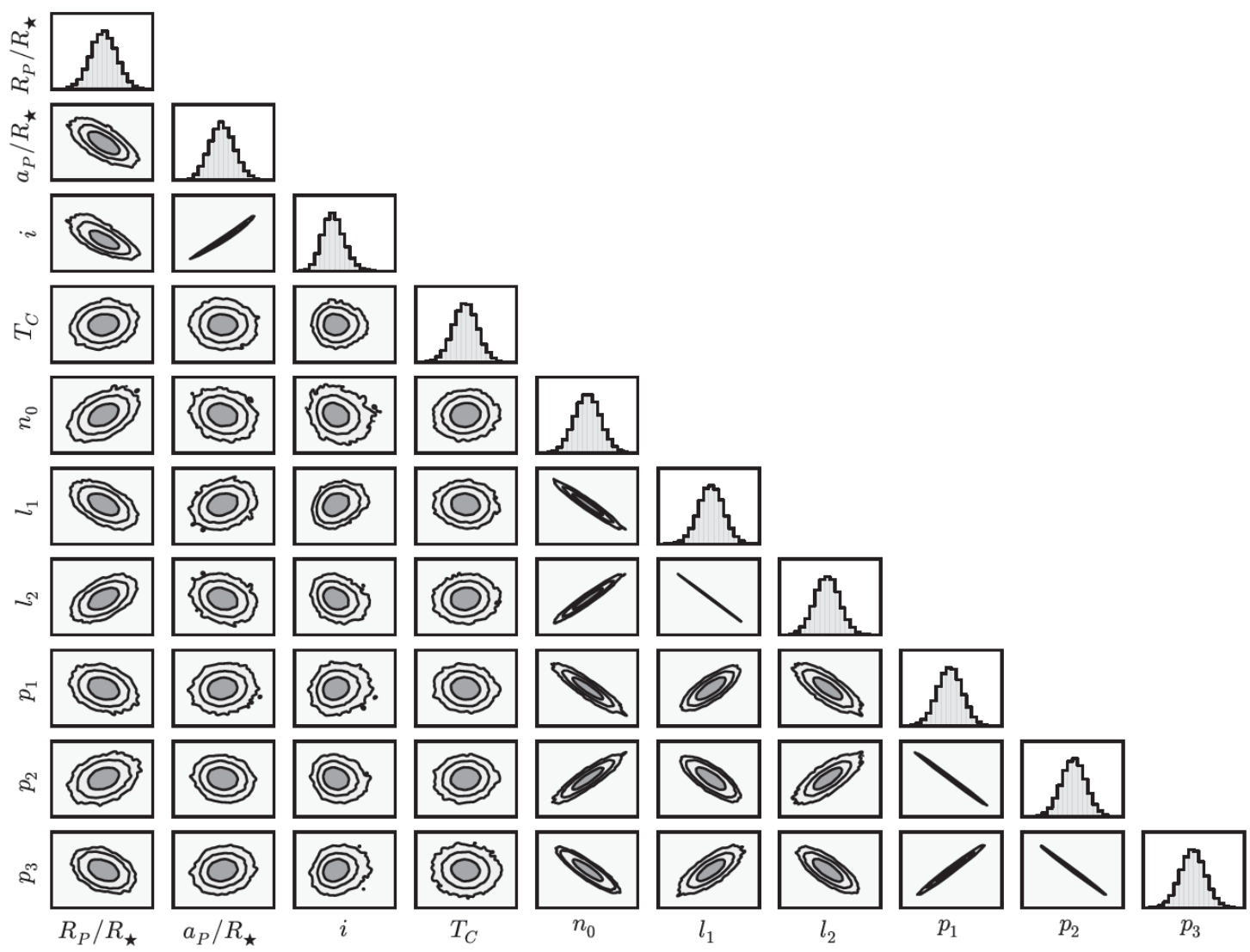

Figure 2.11: Correlation plots of the posterior parameter distributions for the 'white' light curve from MCMC. The lines indicate the areas encompassing $1 \sigma, 2 \sigma$ and $3 \sigma$ of the whole distribution, respectively.

too large transit depth giving us a false result. Another possibility, however, that could explain the discrepancy could be the existence of a strong absorber in the red part of our investigated wavelength interval, which does not overlap the Cousins $I$ band. The occurrence of such an absorber would lift the overall transit depth of our band integrated transit light curve in respect to the Cousins $I$ band measurement. We will investigate if this is the case in the following Sect. 2.5.7 by analyzing the fourteen individual $20 \mathrm{~nm}$ wide channel light curves.

\subsubsection{Multi component noise signal}

The noise model discussed above treats the noise as a smooth function. While this approach gives a good first order correction, the left over red noise still dominates the parameter uncertainties. We have established before that the systematics are not identical for each wavelength channel. Considering the nature of the source of the instrument systematics, it is, however, possible that the signal affecting each curve is identical and only the amplitude of the signal changes. The treatment of suchlike noise signals was discussed by Thatte et al. (2010) and Waldmann (2012) who suggest that systematic noise in multicolor transit data can be described as a linear combination of a limited number of statistically independent signals that 


\begin{tabular}{|c|c|c|c|c|c|}
\hline Parameter & This work (ladc2 paran3) & $\begin{array}{c}\text { B14 } \\
\text { Sloan r' band } \\
540-693 \mathrm{~nm} \\
\end{array}$ & $\begin{array}{c}\text { S12 } \\
\text { Bessel } R \text { band } \\
553-827 \mathrm{~nm} \\
\end{array}$ & $\begin{array}{c}\text { A10 (case } 3) \\
\text { Cousins I band } \\
715-886 \mathrm{~nm} \\
\end{array}$ & $1114-1648 \mathrm{~nm}$ \\
\hline$R_{\mathrm{p}} / R_{\star}$ & $0.13498_{-0.0031}^{+0.0024}$ & $0.13343_{-0.002}^{+0.002 a}$ & $0.1255_{-0.0007}^{+0.0007}$ & $0.12954_{-0.00104}^{+0.00100 b}$ & $0.12316_{-0.00058}^{+0.00058}$ \\
\hline$\delta=\left(R_{\mathrm{p}} / R_{\star}\right)^{2}[\%]$ & $1.82199_{-0.08197}^{+0.06589}$ & $1.78030_{-0.05296}^{+0.05376}$ & $1.57502_{-0.01752}^{+0.01762}$ & $1.678_{-0.027}^{+0.026}$ & $1.51684_{-0.01425}^{+0.01432}$ \\
\hline$a_{\mathrm{p}} / R_{\star}$ & $6.925_{-0.301}^{+0.412}$ & $7.097_{-0.015}^{+0.015 c}$ & $6.959_{-0.371}^{+0.3940 d}$ & $6.95887_{-0.40776}^{+0.40776 d}$ & $6.96_{-0.0220}^{+0.0220 e}$ \\
\hline$i$ & $86.89_{-0.94}^{+1.49}$ & $86.92_{-0.04}^{+0.04}$ & $86.71_{-0.3}^{+0.3}$ & $86.95_{-0.63}^{+0.87}$ & $86.71{\text { (fixed })^{e}}^{-}$ \\
\hline$P[\mathrm{~d}]$ & 3.7354845 (fixed) & $3.7354380(68)$ & $3.7354845(19)$ & $3.73544(74)$ & $3.7354845{\text { (fixed })^{e}}^{e}$ \\
\hline$T_{\mathrm{C}}[\mathrm{d}]$ & $0.65326_{-0.00061}^{+0.00059}$ & - & $0.6533_{-0.0010}^{+0.0010 f}$ & $0.63941_{-0.0028}^{+0.00028 f}$ & $0.65073_{-0.00087}^{+0.00087 f}$ \\
\hline$e$ & 0.0 (fixed) & 0.0 (fixed) & 0.0 (fixed) & 0.0 (fixed) & 0.0 (fixed) \\
\hline$u_{1}$ & 0.2396 (fixed) & 0.2440 (fixed) & n. a. & n.a. & $0.083_{-0.031}^{+0.031}$ \\
\hline$u_{2}$ & 0.2489 (fixed) & $0.21_{-0.02}^{+0.02}$ & n. a. & n.a. & $0.256_{-0.046}^{+0.046}$ \\
\hline
\end{tabular}

Table 2.3: Best-fit planet system parameters from $\chi^{2}$ optimization and confidence intervals from MCMC sampling of the posterior parameter distributions for the 'white' light curves. Also given are the results from the two most recent high precision broadband studies in neighboring and overlapping wavelength bands S12 (Southworth et al. 2012), B14 (Bento et al. 2014) , A10 (Anderson et al. 2010) and M13 (Mandell et al. 2013). $T_{\mathrm{C}}$ is in units of BJD - 2455724. The used red noise factor is $\beta=3.365$. Values without error bars were fixed during the fit.

(a) value calculated from $R_{\star} / a_{\mathrm{p}}$ and $R_{\mathrm{p}} / a_{\mathrm{p}}$ values

(b) value calculated from transit depth $\delta=\left(R_{\mathrm{p}} / R_{\star}\right)^{2}[\%]$

(c) value calculated from $a_{\mathrm{p}}$ and $R_{\star}$ values

(d) value calculated from $R_{\star} / a_{\mathrm{p}}$ value

(e) value cited as adapted from S12

(f) value calculated from the ephemerides given in the respective paper 


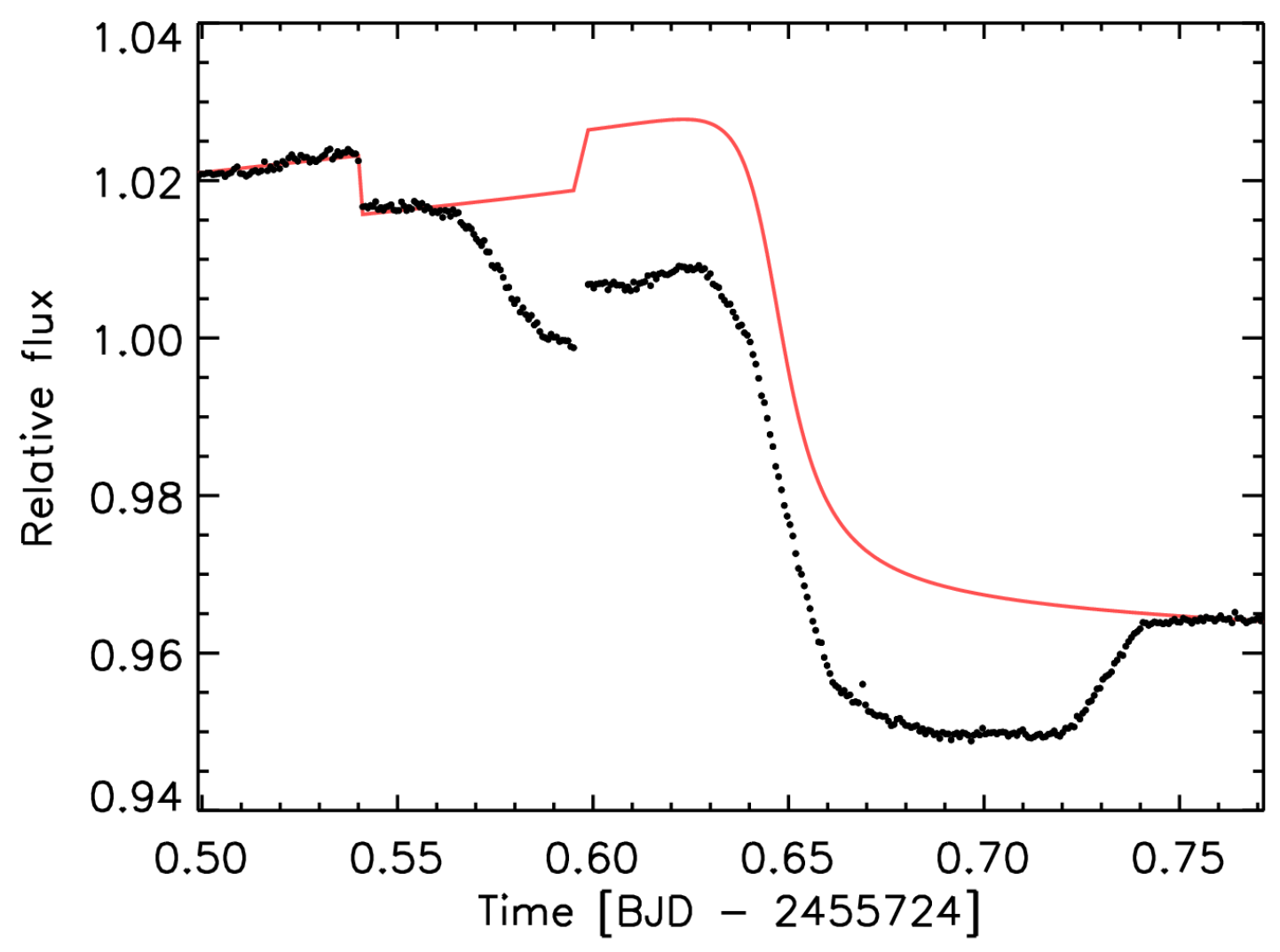

Figure 2.12: 'White light' curve with the best-fit model for the instrument systematics.

affect each wavelength channel with an individual weight. In their approach these signals are identified by using computational signal processing methods. We used the MATLAB implementation of the suggested MULTI-COMBI algorithm (Tichavský et al. 2008), a code that combines the fast Independent Component Analysis code EFICA (Koldovský et al. 2006), designed to separate non-Gaussian, instantaneously mixed signals, and WASOBI (Yeredor 2000), a code optimized for separating Gaussian auto-regressive and time-correlated components. In the trials with our data we did, however, not manage to disentangle transit signal from noise signals by the use of any of these algorithms. We, therefore, decided to create our signal empirically from the results of the 'white' light curve fit. The model $\mathcal{M}$ for the "white' light curve was of the form of:

$$
\mathcal{M}=\mathcal{T} \cdot\left(n_{0}+l_{1} \delta_{\mathrm{LADC}}+l_{2} \delta_{\mathrm{LADC}}^{2}+\mathcal{S}_{\mathrm{PA}}\right)
$$

where

$$
\mathcal{S}_{\mathrm{PA}}=p_{1} \theta_{\mathrm{PA}}+p_{2} \theta_{\mathrm{PA}}^{2}+p_{3} \theta_{\mathrm{PA}}^{3}
$$

and $\mathcal{T}$ is the transit model. We calculate an improved signal $\mathcal{S}_{\text {improved }}$ to replace the previous parameterized model of the paralactic angle dependent model terms. We yield this improved signal using the 'white' light curve data $\mathcal{D}_{\text {white light }}$ and the best-fit parameters of the 'white' light curve fit. In the following $T^{*}$ indicates a transit model calculated from the best-fit 'white' light parameters given in Table 2.3 


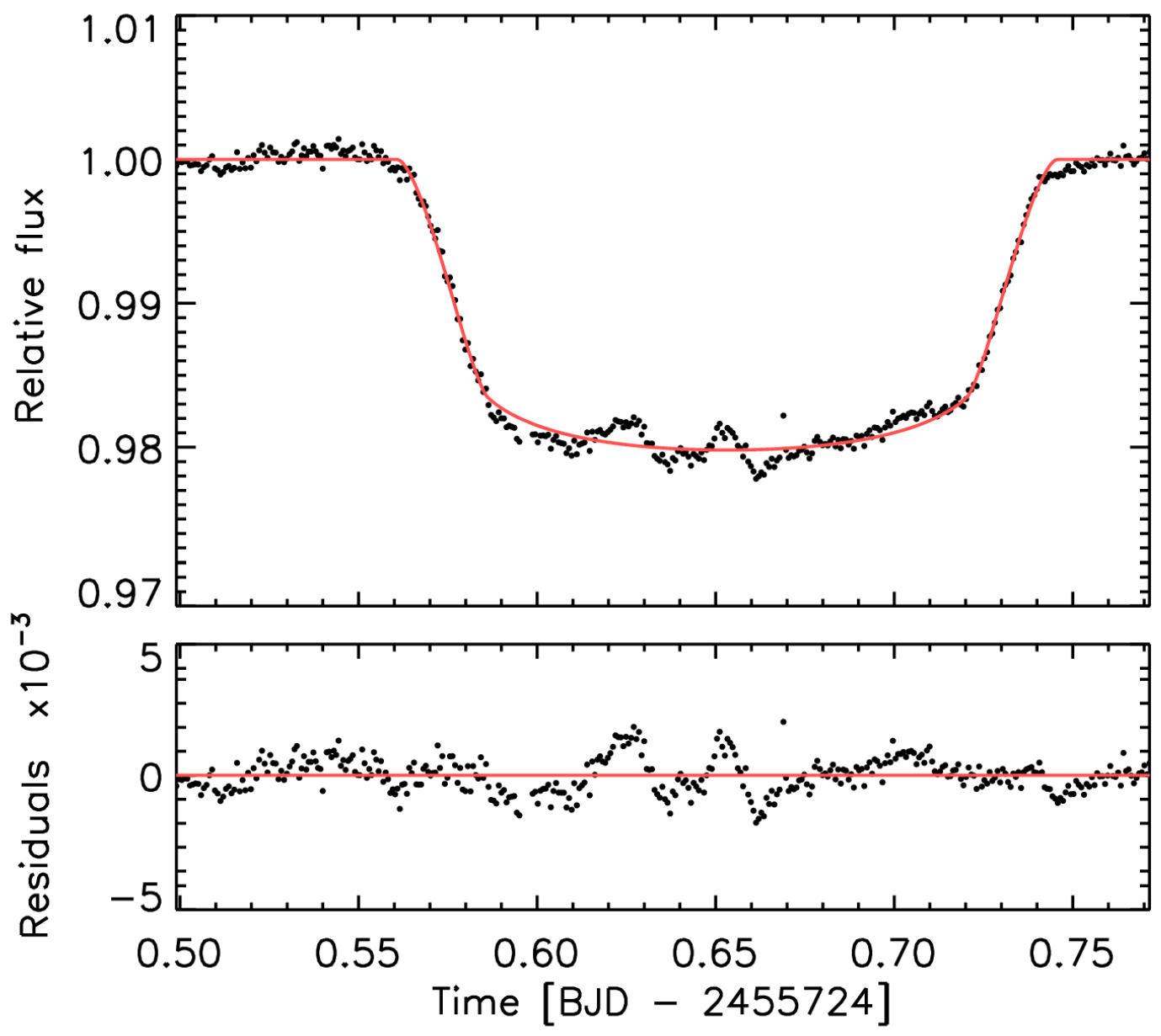

Figure 2.13: Top panel: 'white light' curve corrected from instrument systematics with the best-fit model for the transit shape. Bottom panel: residuals of the original light curve after the best-fit model was subtracted.

and $n_{0}^{*}$ and $l_{1,2}^{*}$ the best-fit parameters for the systematic trend.

$$
\left.\mathcal{S}_{\text {improved }}=\left(\mathcal{D}_{\text {white light }} / \mathcal{T}^{*}\right)-n_{0}^{*}-l_{1}^{*} \delta_{\text {LADC }}-l_{2}^{*} \delta_{\text {LADC }}^{2}\right)
$$

The improved signal $\mathcal{S}_{\text {improved }}$ is shown in Fig. 2.15 (blue circles) together with the paralactic angle dependent part $\mathcal{S}_{\mathrm{PA}}$ of the best-fitting smooth analytical systematics model (black line) and the flat field rotation pattern transmittance curve (red triangles) for comparison. This signal can now be used to model the data of individual wavelength channels with the new model approach $\mathcal{M}_{\text {improved, }}$ where $s_{1}$ is a new free parameter, scaling the signal individually for every narrowband light curve. This approach is very similar to the recently independently developed 'Divide-White' method used by Stevenson et al. (2014b) and Kreidberg et al. (2014).

$$
\mathcal{M}_{\text {improved }}=\mathcal{T} \cdot\left(n_{0}+l_{1} \delta_{\mathrm{LADC}}+l_{2} \delta_{\mathrm{LADC}}^{2}+s_{1} \mathcal{S}_{\text {improved }}\right)
$$

A possible weakness of this approach of creating an improved instrumental sys- 


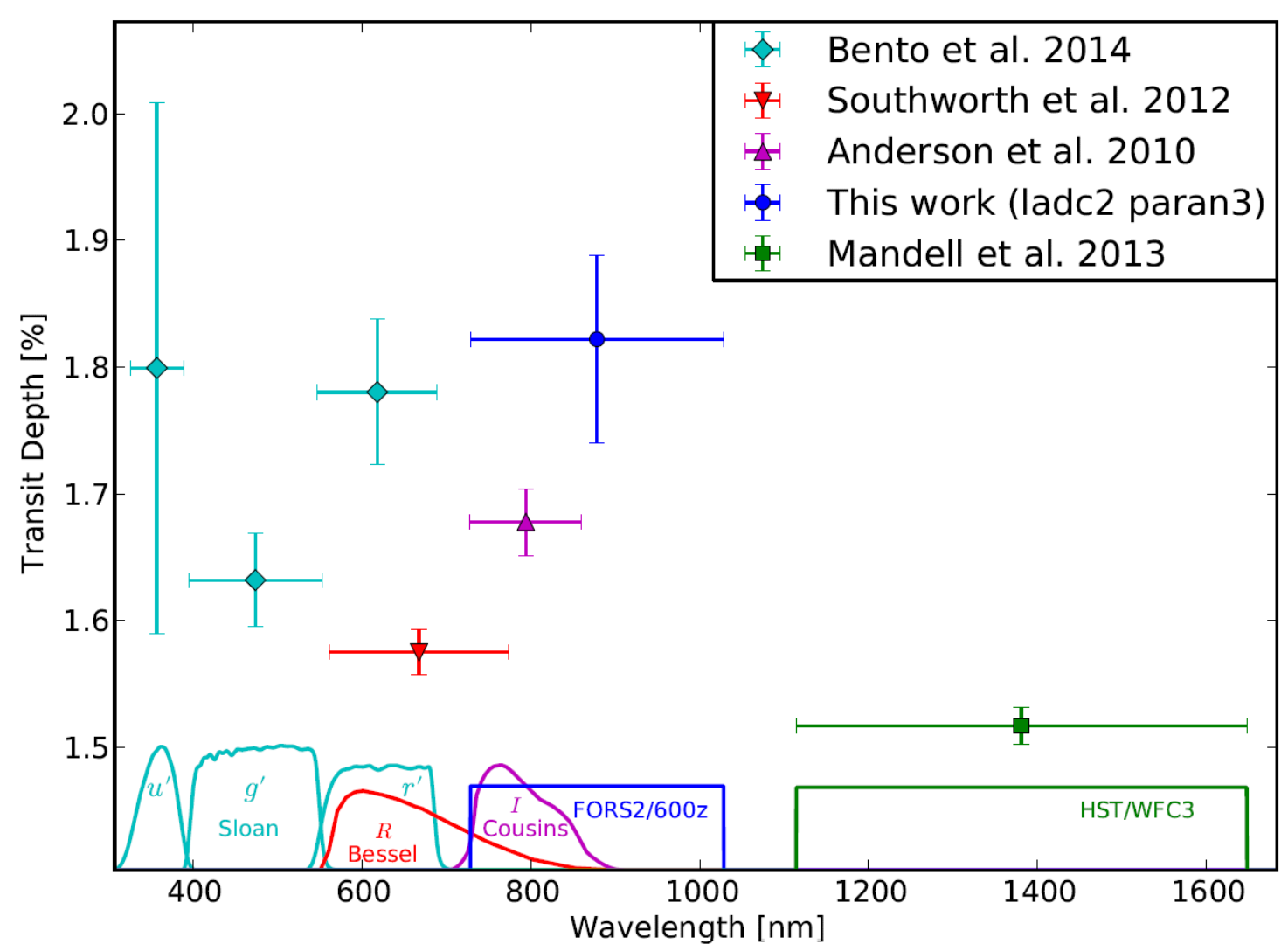

Figure 2.14: The transit depth of our observation of WASP-17 and literature values. Also plotted on the bottom are the filter transmittance curves and wavelength intervals probed by the spectrophotometric studies.

tematics signal could be that we have to assume the previously determined orbital parameters and especially the transit depth of the 'white' light curve to be absolutely correct. Since we used a smooth model to correct a non-smooth instrument systematic signal to derive our best-fit 'white' light curve values, we do not have sufficient grounds for such absolute faith in our best-fit values. To investigate the effect using flawed values could potentially have we calculated three alternative improved instrumental systematics signals $\mathcal{S}_{\text {improved }}^{\mathrm{a}, \mathrm{b}, \mathrm{c}}$ using each of the three literature parameter sets given in Table 2.3. As an example the signal we yield when using the values from Mandell et al. (2013) is shown in Fig. 2.15 (green squares). We investigate and discuss the impact of using those signals instead of the one based on our best-fit parameters in Sect. 2.5.7.

\subsubsection{Transmission spectrum}

In the next step we derive the wavelength dependent planet radius by fitting each of the fourteen $20 \mathrm{~nm}$ wide narrowband channel light curves individually. As discussed in the previous Sect. we now use a model of the form given in equation 2.8 consequently exchanging the terms of the model, which were dependent on the paralactic angle $\theta_{\mathrm{PA}}$ with an empirically gained signal $\mathcal{S}_{\text {improved }}$ based on our best-fit 'white' 


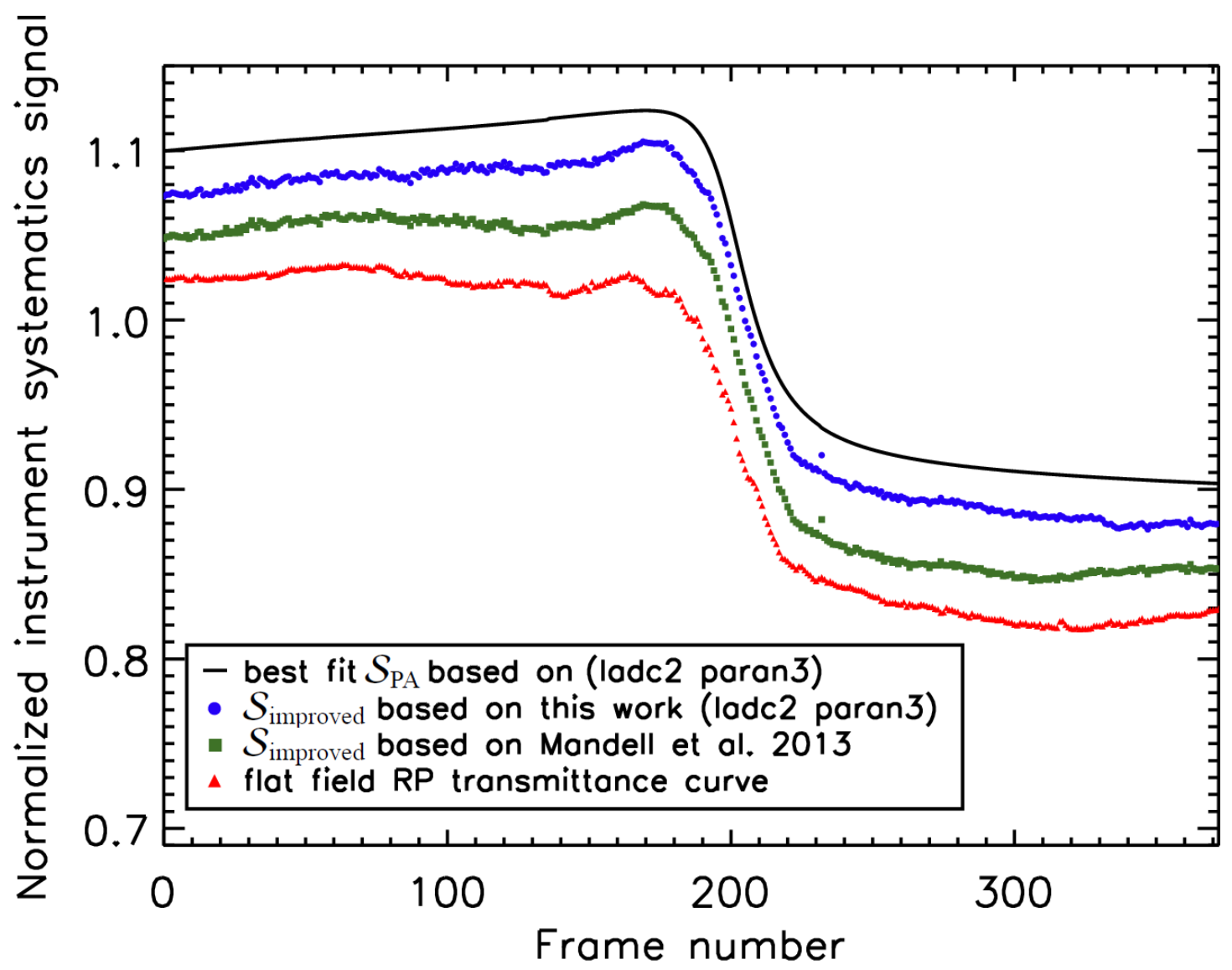

Figure 2.15: Empirically gained signal $\mathcal{S}_{\text {improved }}$ of the instrument systematics affecting the 'white' light curve (blue circles). We use scaled version of this signal to correct the instrument systematics affecting the individual narrowband light curves. For comparison also the paralactic angle dependent part of the best-fitting analytical systematics model $\mathcal{S}_{\mathrm{PA}}$ (black line), the empirical signal $\mathcal{S}_{\text {improved we yield when }}$ fixing the 'white' light transit parameter to the values from Mandell et al. (2013) (green squares) and the scaled transmittance curve we extracted from the flat field rotation pattern (red triangles) are shown. We applied uniform offset to the last mentioned tree curves for better visual clarity. 
light curve parameters given in Table 2.3. Furthermore, we keep all planet system parameters that can be considered wavelength independent (i.e. all parameters except the radius ratio $R_{\mathrm{p}} / R_{\star}$ and the limb darkening coefficients $u_{1}, u_{2}$ ) fixed to the best-fit values from the 'white' light curve fit given in Table 2.3. While the limb darkening parameters had to be kept fixed in the 'white' light curve fit, the use of an empirical signal $\mathcal{S}_{\text {improved }}$ significantly reduced the red noise level responsible for the un-physical best-fit values calculated when the limb darkening was left free. Consequently, it was possible to allow the limb darkening to be optimized during the fit of the narrowband channel light curves. As both parameters exhibit a certain degree of degeneracy, we decided to keep the linear parameter $u_{1}$ fixed to the theoretical value calculated from the PHOENIX atmospheres and only allowed the quadratic value $u_{2}$ to vary. The parameter always converged to a physical value for all fourteen curves and did not show any large discrepancies to the prediction. We again found the best-fit values by using the Levenberg-Markward algorithm, and derived the confidence intervals from sampling the posterior parameter distributions with MCMC like in the case of the 'white' light curve. As an example the correlation plots for the posterior parameter distributions of channel \#13 (978 nm - $998 \mathrm{~nm}$ ) are given in Fig. 2.16. Although the red noise was strongly reduced due to using the empirically generated signal $\mathcal{S}_{\text {improved }}$ the behavior of the residuals when binned in time still deviated slightly from the expected curve of pure white noise for some of the narrowband light curves. We, therefore, also repeated the step of calculating the 'red noise factor' $\beta$ for each channel light curve, and inflated the error bars derived from the MCMC accordingly. All 14 light curves corrected from the instrumental systematics and plotted together with the best-fit model are shown in Fig. 2.17.

When studying the results for the wavelength dependent transit depth we can see a clear large scale trend of rising planetary radii i.e. larger transit depth towards shorter wavelengths for WASP-17b. In the previous Sect. we stated that the offset between our band integrated transit depth and the Cousins $I$ band measurement by (Anderson et al. 2010) might be explained if we detect a strong absorption in the red part of the probed wavelength that does not affect the blue, Cousins $I$ band overlapping, part. Our results for the transmission spectrum, however, point towards the opposite scenario, hinting at a strong absorber in the blue part of the probed wavelength interval. Therefore, we have to consider the second mentioned explanation for the discrepancy between our measurement and the Cousins $I$ band, which is that our approach to modeling the instrument systematic as a smooth function is insufficient in differentiating low-amplitude systematic noise from transit depth signal causing us to obtain a slightly too large transit depth result for the white light curve.

\subsubsection{Test of the empirical 'systematics signal' approach}

As mentioned before in Sect. 2.5.6 we have to test if the use of the empirically gained improved instrument systematics signal $\mathcal{S}_{\text {improved }}$ is flawed due to having to assume a set of 'white' light orbital and planet parameters as fixed neglecting their inherent uncertainties. To explore effect that the introduced 'white' light curve parameters have on the narrowband channel results, therefore, repeated the calculation of an 
empirical noise signal and all steps of the channel light curve fitting three times, in each case assuming one of the three sets of literature parameters cited in Table 2.3 instead of our own best-fit 'white' light curve values. For all three cases the empirical noise signal was computed as before by using Eq. 2.8 with the alteration that now the theoretical transit curve $\mathcal{T}^{*}$ was calculated using one the respective set of literature values. The only transit light curve parameters we did not adopt from the literature for this calculation were the midtransit time $T_{\mathrm{C}}$ which we kept fixed to our own best-fit value and the two limb darkening parameters, which we keep fixed in each case to the theoretical values we previously derived from the PHOENIX models for the precise probed wavelength interval.

From this test we identified that the main impact of altering the assumed 'white' light curve parameters is a shift in the value around which the transit depths of the fourteen individual channels fluctuate. When studying the differential change of the transit depth with respect to the assumed 'white' light curve transit depth the result was almost identical for all tested cases. The difference in the remaining orbital parameters between the individual test cases did not appear to have any affect at all.

Between the tested cases we found the largest discrepancy in transit depth variation occurring for the bluest and the reddest channel. This can be easily explained when looking at the wavelength dependent behavior of the parameter $s_{1}$, which is scaling the respective empirical 'improved systematics signal' $\mathcal{S}_{\text {improved. }}$ As such it will not only scale the true instrument systematic signal but also a potential excess or lack of transit depth that was erroneously incorporated in it. We find that only for the bluest and the reddest channel light curve this parameter significantly differs from 1 , causing the simultaneously up or down scaled erroneously incorporated transit depth of the empirical signal to alter the channels best-fit transit depth result. As we would expect from this line of thought, we find the difference of the transit depth variations in these two channels to be most prominent between the results we gain when using our best-fit parameters (i.e. the largest of all tested 'white' light curve transit depths) and the literature values given by Mandell et al. (2013) (i.e. the smallest of all tested 'white' light transit depths). Still, both results for the relative transit depth variation are comparable within their error bars (see Fig. 2.18). We conclude that while we cannot give the absolute transit depth with confidence, our approach to derive the wavelength dependent variation of the transit depth is robust under different assumed orbital parameters. We, therefore, give our final results in Table 2.4 as the differential transit depth with respect to the 'white' light curve transit depth. For this we use the best-fit values we yielded from using our own best-fit transit parameters but as error bars we adopt the largest and the lowest value of the $1 \sigma$ uncertainty intervals of all tested cases respectively. This mainly affects the bluest and reddest channel, resulting in a larger uncertainty towards larger transit depth for the bluest channel and a larger uncertainty towards smaller transit depths for the reddest channel. This variation and the error bars are in this form valid in a range of possible 'white' light transit depths between $1.517 \%$ and $1.822 \%$. To convert the values into absolute transit depth a 'white' light transit depth lying within this interval has to be added to the values. In our optimization of all channel light curves, we also obtain best-fit values for the linear limb darkening parameter 

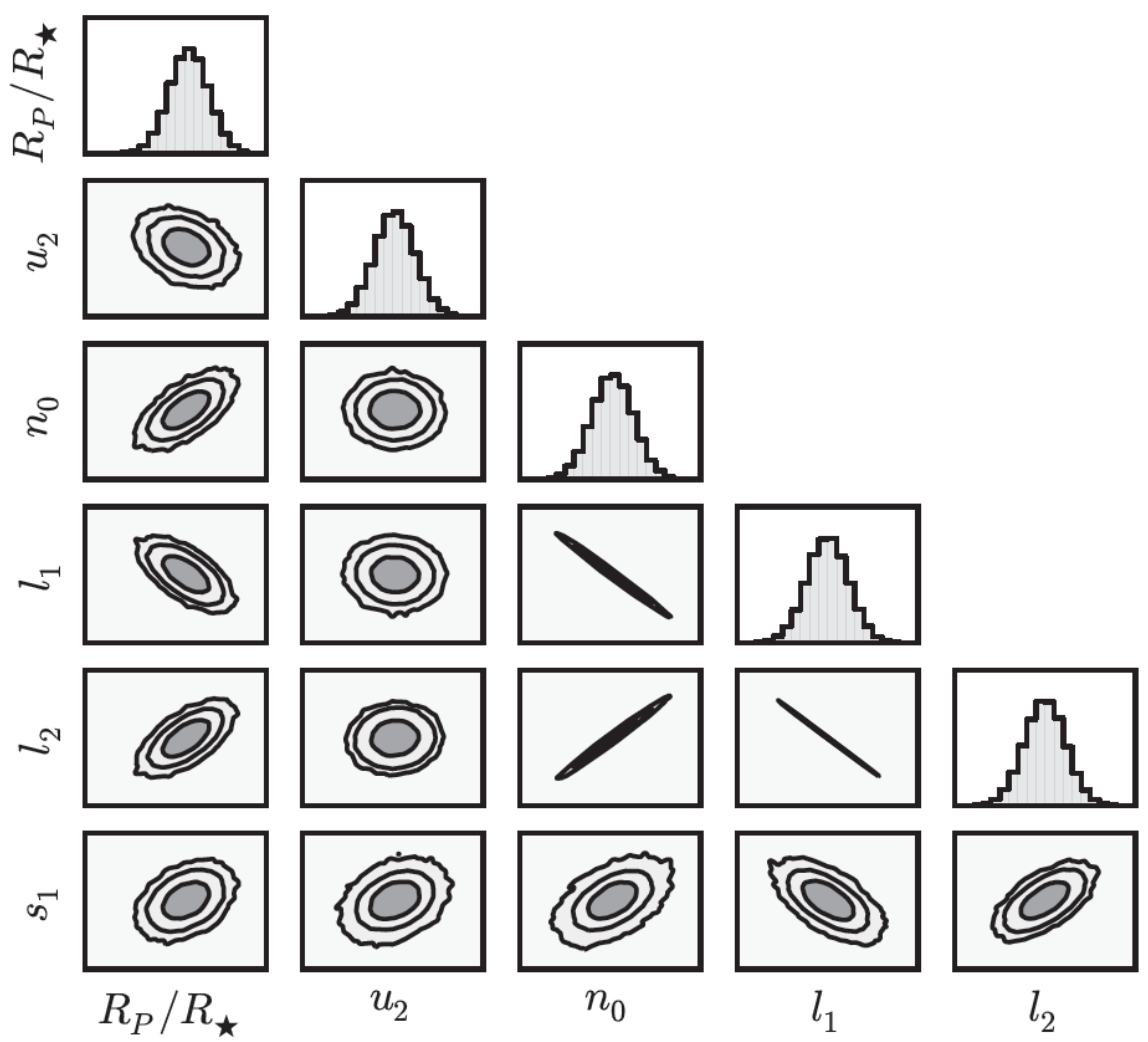

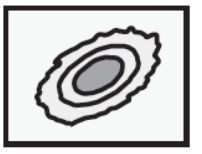

$n_{0}$

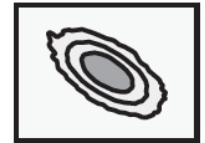

$l_{1}$

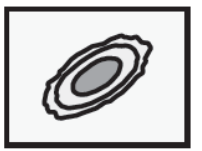

$l_{2}$

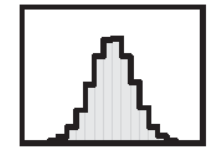

$s_{1}$

Figure 2.16: Example correlation plots for the posterior parameter distributions of the narrowband channel \#13 (978 nm - $998 \mathrm{~nm})$. The lines indicate the areas encompassing $1 \sigma, 2 \sigma$ and $3 \sigma$ of the whole distribution, respectively.

$u_{2}$. We compare the results for all tested cases of 'white' light curves parameter sets and find that also here the best-fit values are consistent for all tested cases. They, furthermore, are sufficiently consistent with the theoretical prediction (see Fig. 2.19). The resulting parameters for $u_{2}$, also with extend uncertainty intervals, are listed in Table 2.4 together with the results for the transit depth and the red noise factor $\beta$. 


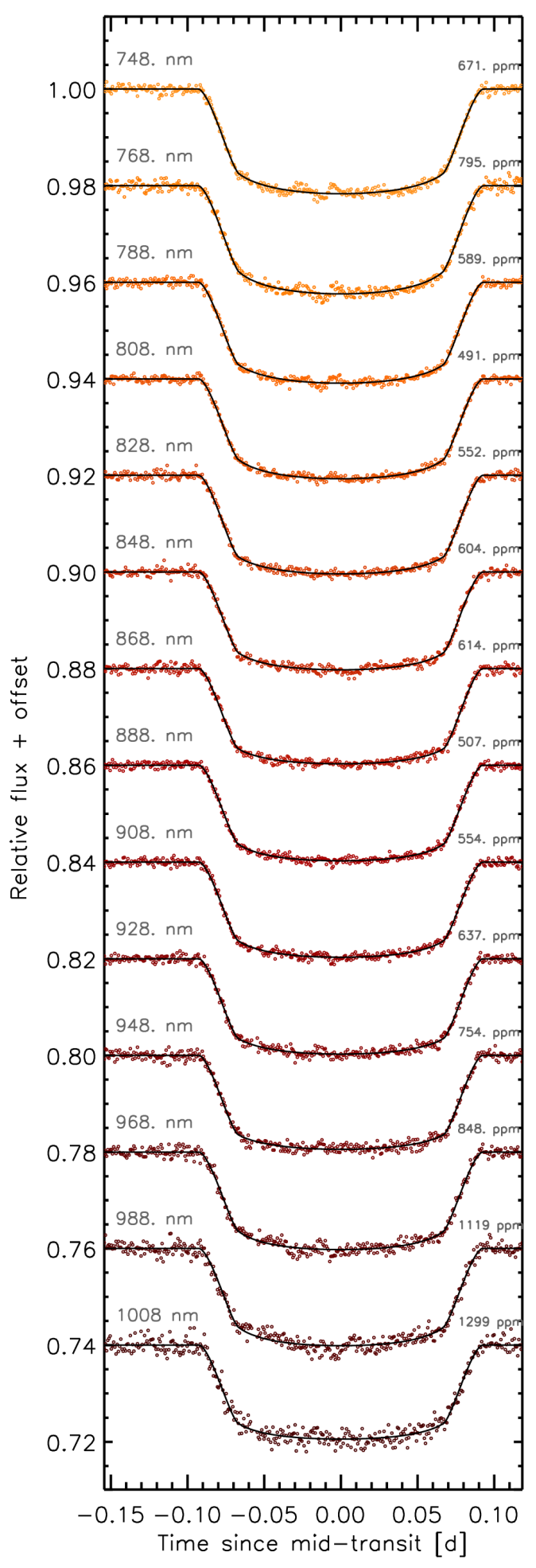

Figure 2.17: All 14 light curves corrected from the instrumental systematics and plotted together with the best-fit model. 


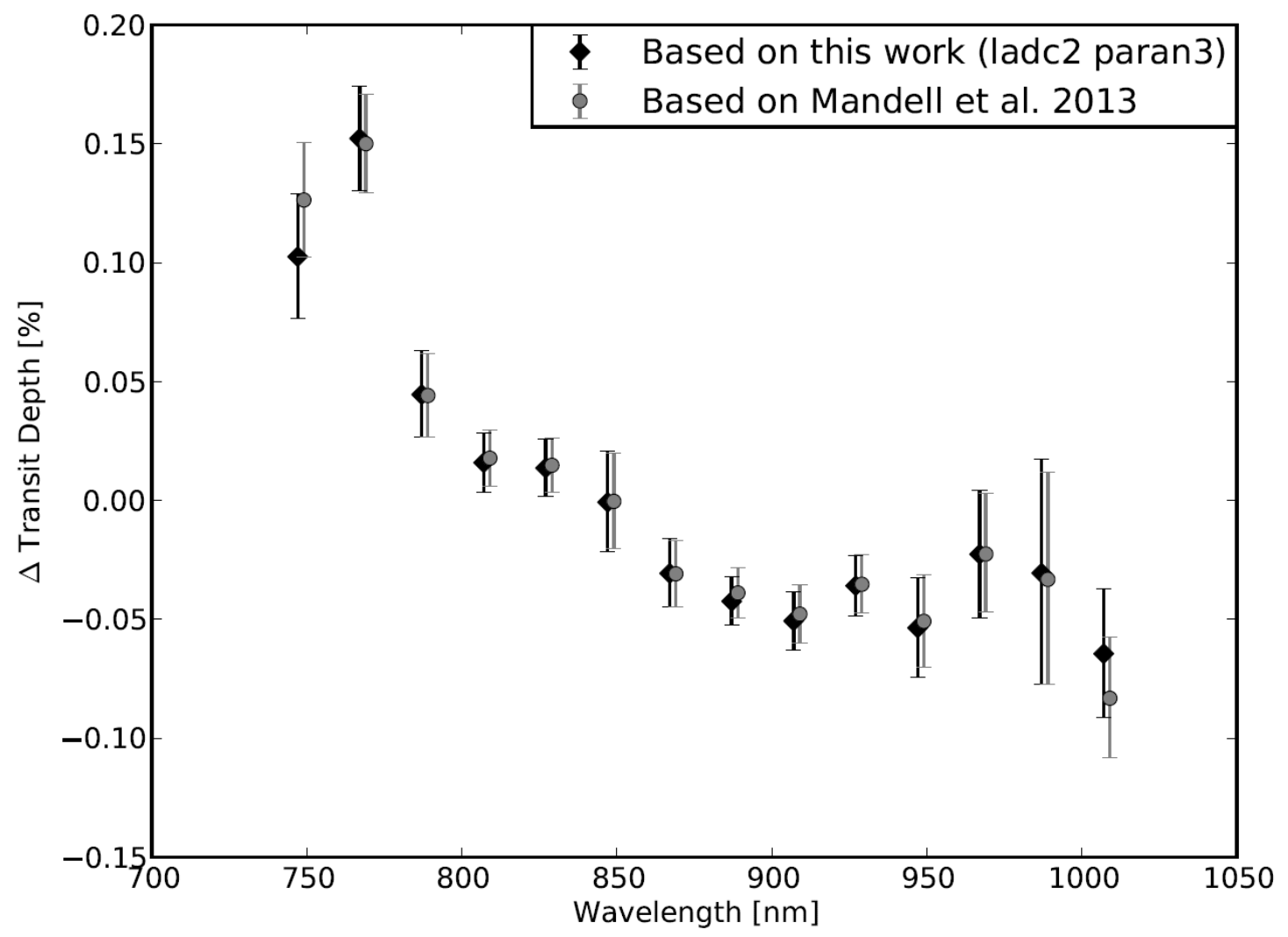

Figure 2.18: Differential transit depth of WASP-17b with respect to the assumed transit depth of the 'white' light curve for the two different analyses based on different assumptions for the orbital parameters and transit depth of the 'white' light curve. Shown are the two most extreme cases: largest and smallest 'white' light curve transit depth. The fit to our data with the instrument systematics correction model 'ladc2 paran3' yields the largest transit depth measured for this planet so far. The smallest transit depth was found by Mandell et al. (2013).

\subsection{Comparison to literature data and theoretical models}

To enable the comparison with literature data we require an absolute transit depth rather than our previously given relative transit depth variation. For this purpose we decided to add a baseline transit depth of $\delta=1.621$ to the relative transit depth, rather than our best-fit 'white' light curve transit depth. The used value ensures consistency of our narrowband channel transit depth results and the Cousins $I$ band measurement by Anderson et al. (2010). Considering, the uncertainty of our absolute transit depth determination due to the strong impact of the instrument systematics on our data we consider this literature value the more reliable reference. Consistency of our results and the Cousins $I$ band measurement in this case means, that after adding the baseline transit depth $\delta=1.621$, the calculation of theoretical Cousins $I$ band measurement from our narrowband light curve transit depth results yields a value identical to the one from Anderson et al. (2010). The baseline transit depth 


\begin{tabular}{lcccccc}
\hline \hline \multicolumn{3}{l}{ Wavelength bin limits [nm] } & $u_{1}$ (fixed) & $u_{2}$ & $\Delta$ Transit depth [\%] & Red noise factor $\beta$ \\
\hline 738 & - & 758 & 0.29732 & $0.20767_{-0.0608}^{+0.04532}$ & $0.10253_{-0.02647}^{+0.04793}$ & 2.317 \\
758 & - & 778 & 0.28536 & $0.26897_{-0.03712}^{+0.05666}$ & $0.15222_{-0.02300}^{+0.02176}$ & 1.611 \\
778 & - & 798 & 0.27415 & $0.21045_{-0.03646}^{+0.04098}$ & $0.04454_{-0.01836}^{+0.01786}$ & 1.824 \\
798 & - & 818 & 0.25779 & $0.26417_{-0.02247}^{+0.03277}$ & $0.01592_{-0.01258}^{+0.01363}$ & 1.500 \\
818 & - & 838 & 0.23542 & $0.23660_{-0.03124}^{+0.02235}$ & $0.01362_{-0.01207}^{+0.01269}$ & 1.294 \\
838 & - & 858 & 0.21550 & $0.27154_{-0.04999}^{+0.04267}$ & $-0.00077_{-0.02136}^{+0.02065}$ & 2.069 \\
858 & - & 878 & 0.20575 & $0.23040_{-0.04256}^{+0.02687}$ & $-0.03063_{-0.01458}^{+0.01401}$ & 1.361 \\
878 & - & 898 & 0.20996 & $0.28152_{-0.02436}^{+0.02421}$ & $-0.04239_{-0.01032}^{+0.01389}$ & 1.202 \\
898 & - & 918 & 0.21443 & $0.27030_{-0.03180}^{+0.0239}$ & $-0.05064_{-0.01236}^{+0.01509}$ & 1.330 \\
918 & - & 938 & 0.22935 & $0.21299_{-0.03598}^{+0.02433}$ & $-0.03586_{-0.01284}^{+0.01275}$ & 1.191 \\
938 & - & 958 & 0.22531 & $0.19017_{-0.06047}^{+0.0445}$ & $-0.05355_{-0.02117}^{+0.02219}$ & 1.652 \\
958 & - & 978 & 0.21610 & $0.32074_{-0.05060}^{+0.0630}$ & $-0.02264_{-0.02676}^{+0.02680}$ & 1.870 \\
978 & - & 998 & 0.22066 & $0.32102_{-0.00864}^{+0.11669}$ & $-0.03057_{-0.04809}^{+0.04657}$ & 2.526 \\
998 & - & 1018 & 0.20076 & $0.27185_{-0.05294}^{+0.07828}$ & $-0.06445_{-0.04432}^{+0.02662}$ & 1.224 \\
\hline
\end{tabular}

Table 2.4: Best-fit results for the differential transit depth ( $\Delta$ transit depth [\%]) and limb darkening parameters $u_{1}$, and $u_{2}$ as well as the red noise factor $\beta$ derived for every channel. To convert the differential into an absolute transit depth a baseline transit depth $\delta$ can be added to the results. The values and uncertainty intervals given in this Table have been tested for the interval $1.517 \% \geq \delta \leq 1.822 \%$. For consistency of our results and the Anderson et al. (2010) Cousins $I$ band measurement use $\delta=1.612 \%$ (see Sect. 2.6). 


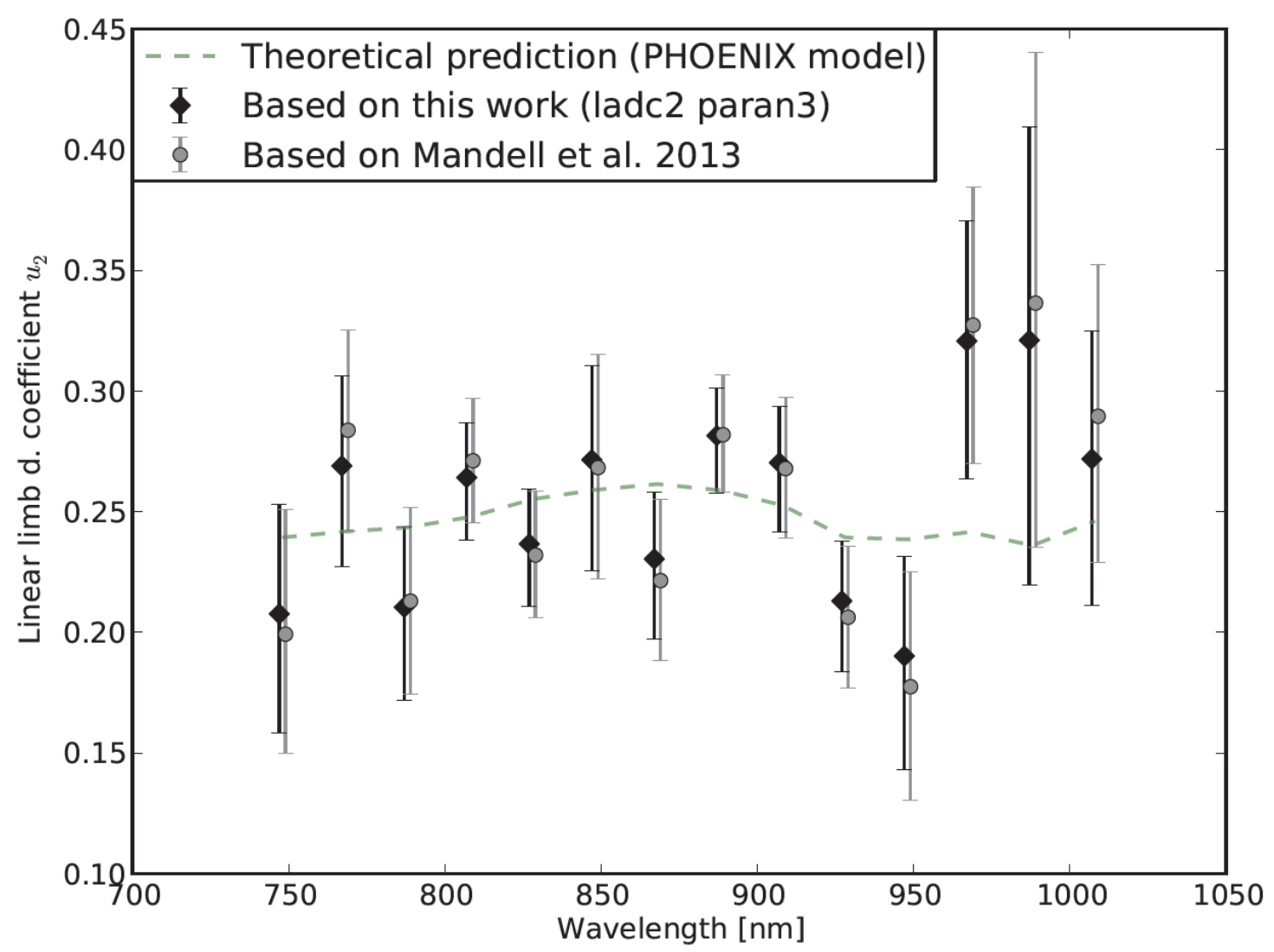

Figure 2.19: Best-fit linear limb darkening coefficient $u_{2}$ for the two different analyses based on different assumptions for the orbital parameters and transit depth of the 'white' light curve.

needed to establish this consistency, lies within the interval for which we tested our relative transit depth results and respective uncertainty intervals to be robust (see Sect. 2.5.7).

After enforcing this consistency, we can see that our data forms the bridge between the two spectroscopic studies conducted in the red and blue neighboring wavelength regions by Mandell et al. (2013) and Bento et al. (2014). The transition from small to large radii that is exhibited by our data and supported by most literature values is roughly expected from theoretical models of solar composition gas (Asplund et al. 2009) in chemical, hydrostatic and radiative-convective equilibrium at the elevated temperatures expected for a planet like WASP-17b (see Fig. 2.20, upper panel). The model was calculated using the PHOENIX model atmosphere code assuming dayside-only $(2 \pi)$ redistribution (Barman et al. 2005) and the transit spectrum was obtained assuming a one-dimensional (1D) spherically symmetric geometry following the procedure of Barman et al. (2003). The model is dominated by $\mathrm{TiO}$ and VO absorption bands, which in the optical strongly contribute to the total opacity. Such models are often referred to as pM class models (Fortney et al. 2008). The model shows an increase in transit depth toward shorter wavelength very similar to the one in our data. This increase in transit depth, however, is caused by strong $\mathrm{TiO} / \mathrm{VO}$ 
absorption, which is expected to occur in several separated broad absorption bands causing a specific structure over the wavelength interval that was probed by us. In contrast to that our results show a rather featureless/smooth increase in transit depth and do not reproduce the absorption bands typically associated with $\mathrm{TiO} / \mathrm{VO}$ (see Fig. 2.20, lower panel). TiO is expected to rain out in cooler atmospheres (often referred to as pL class atmospheres). To explore the possibility that the limb is cooler than a dayside-only redistribution model a second model was computed, in which $\mathrm{TiO}$ and $\mathrm{VO}$ opacity was excluded and the incident stellar flux was lowered by a factor of five resulting in limb temperatures about $500 \mathrm{~K}$ cooler than in the fullredistribution model. If we compare our data to this colder $\mathrm{TiO} / \mathrm{VO}$-free model we find the main model absorption features to be due to potassium (potassium doublet 767 and $769 \mathrm{~nm}$ ) and water (water band roughly between 925 and $1030 \mathrm{~nm}$ ) in an otherwise flat spectrum (see Fig. 2.21, lower panel). Similar features can be found in our data on top of the steep incline. Without an additional underlying mechanism that would cause an increase in transit depth towards shorter wavelength such a cold model is however not only inconsistent with our data but also with all broadband measurements. Figure 2.22 shows the comparison of the transit depth data with a pL class like model in which a slope was added to the wavelength region that was probed in this study. In the following Sects. we investigate possible astrophysical causes for the smooth steep incline we have measured.

\subsubsection{Rayleigh scattering}

In previous studies steep slopes in atmospheric spectra have been associated with Rayleigh scattering (Pont et al. 2008). If we compare our slope ( $\mathrm{d} R_{\mathrm{p}} \approx 7573.10 \mathrm{~km}$ over the wavelength interval of 738 $-1018 \mathrm{~nm}$ ) with Eq. 2.9 taken from Lecavelier Des Etangs et al. (2008, Equation (4)), where $g$ is the planet surface gravity, $m$ is the mean molecular weight of the atmosphere gas and $k_{b}$ is the Boltzmann constant, we yield $\alpha T_{\text {eq }} \approx-20612.07$. Considering $\alpha \approx-4$ is typical for Rayleigh Scattering this would translate into a planet temperature of $T_{\text {eq }} \approx 5153 \mathrm{~K}$. Alternatively using the equilibrium temperature of WASP-17b given by Southworth et al. (2012), $T_{\text {eq }}=$ $1755 \pm 28$, we arrive at a value of $\alpha \approx-11.74$, which leads to the conclusion that Rayleigh scattering is an unlikely cause for the wavelength dependent radius slope. For visual comparison Fig. 2.23 shows a theoretical Rayleigh scattering curve (i.e. $\alpha=-4$ ) for the equilibrium temperature $T_{\text {eq }}=1755 \pm 28$ in context with the data from this and previous studies of WASP-17b atmosphere.

$$
\alpha T_{\mathrm{eq}}=\frac{\mu g}{k_{b}} \frac{\mathrm{d} R_{\mathrm{p}}}{\mathrm{d} \ln \lambda}
$$

\subsubsection{Un-occulted star spots}

Another physical explanation for the observed slope could be the existence of unocculted star spots, i.e. spots of lower temperature residing on a part of the stellar surface which is not crossed by the planet in our line of sight. The occurrence of such spots would offset the baseline of the out of transit light curve, to which the traditional transit model is normalized to, downward causing a larger overall transit 

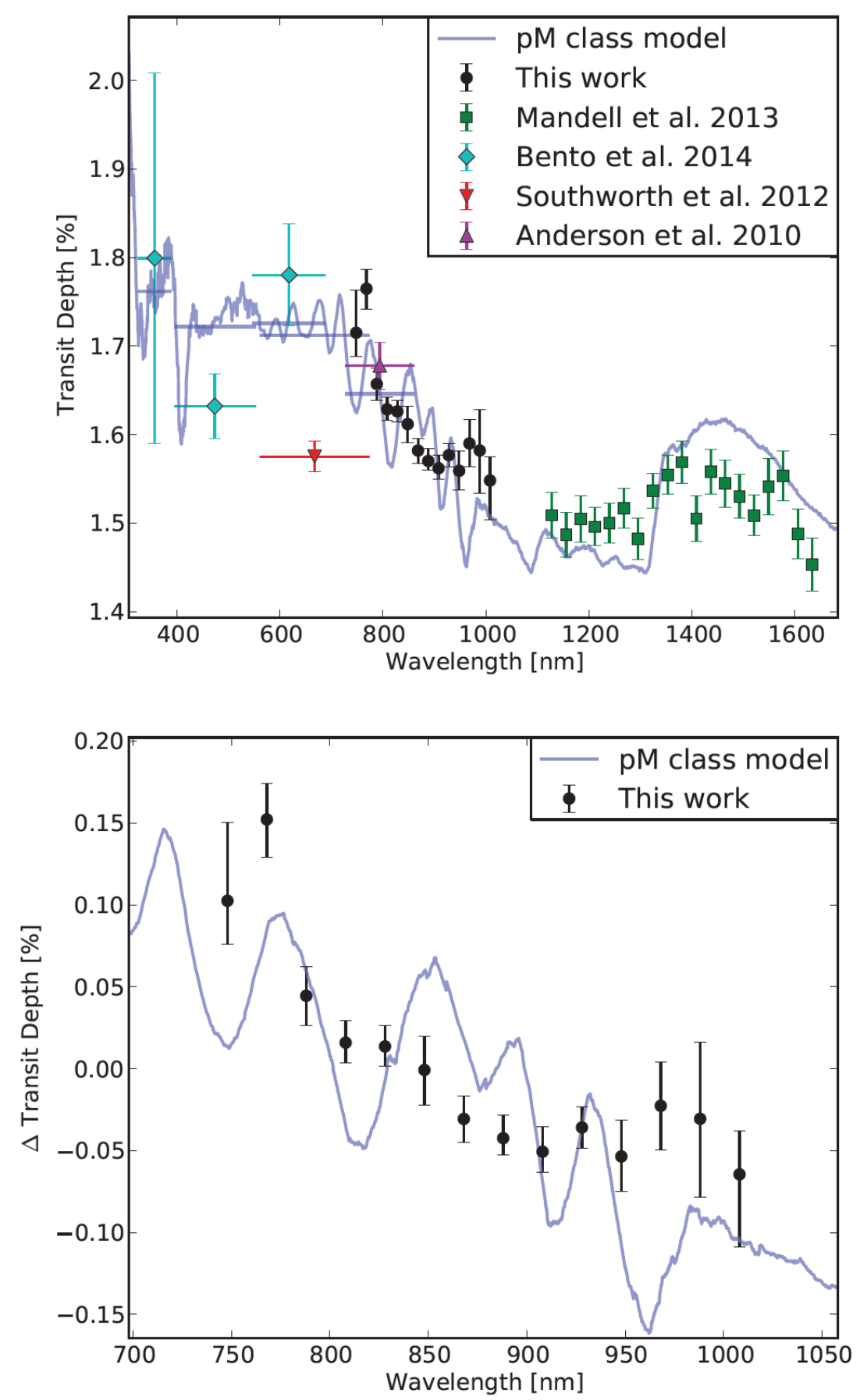

Figure 2.20: Top panel: our data points (circles) set into context with literature values of Bento et al. (2014) (diamonds), Southworth et al. (2012) (downwards arrow), Anderson et al. (2010) (upwards arrow) and Mandell et al. (2013) (squares). A baseline transit depth of $\delta_{\text {white light }}=1.612$ was assumed for our data, enforcing consistency between our data and the Anderson et al. (2010) value. A theoretical pM class like model is shown in blue (solid line). Bottom panel: the relative transit depth variation of our data and the theoretical pM class like model (solid blue line). 

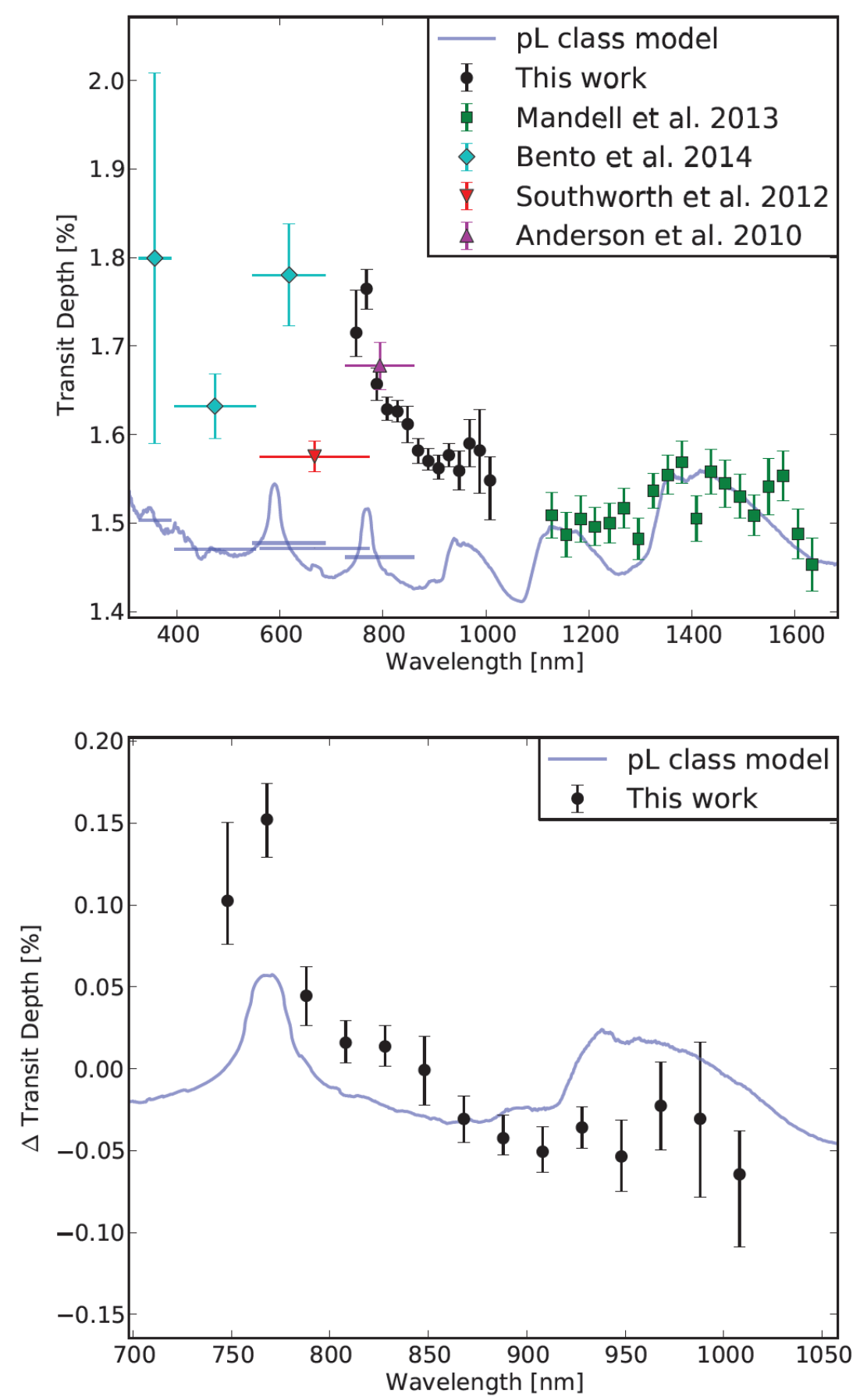

Figure 2.21: Top panel: our data points (circles) set into context with literature values of Bento et al. (2014) (diamonds), Southworth et al. (2012) (downwards arrow), Anderson et al. (2010) (upwards arrow) and Mandell et al. (2013) (squares). A baseline transit depth of $\delta_{\text {white light }}=1.612$ was assumed for our data, enforcing consistency between our data and the Anderson et al. (2010) value. A theoretical $\mathrm{pL}$ class like model is shown in blue (solid line). Bottom panel: the relative transit depth variation of our data and the theoretical pL class like model (solid blue line). 

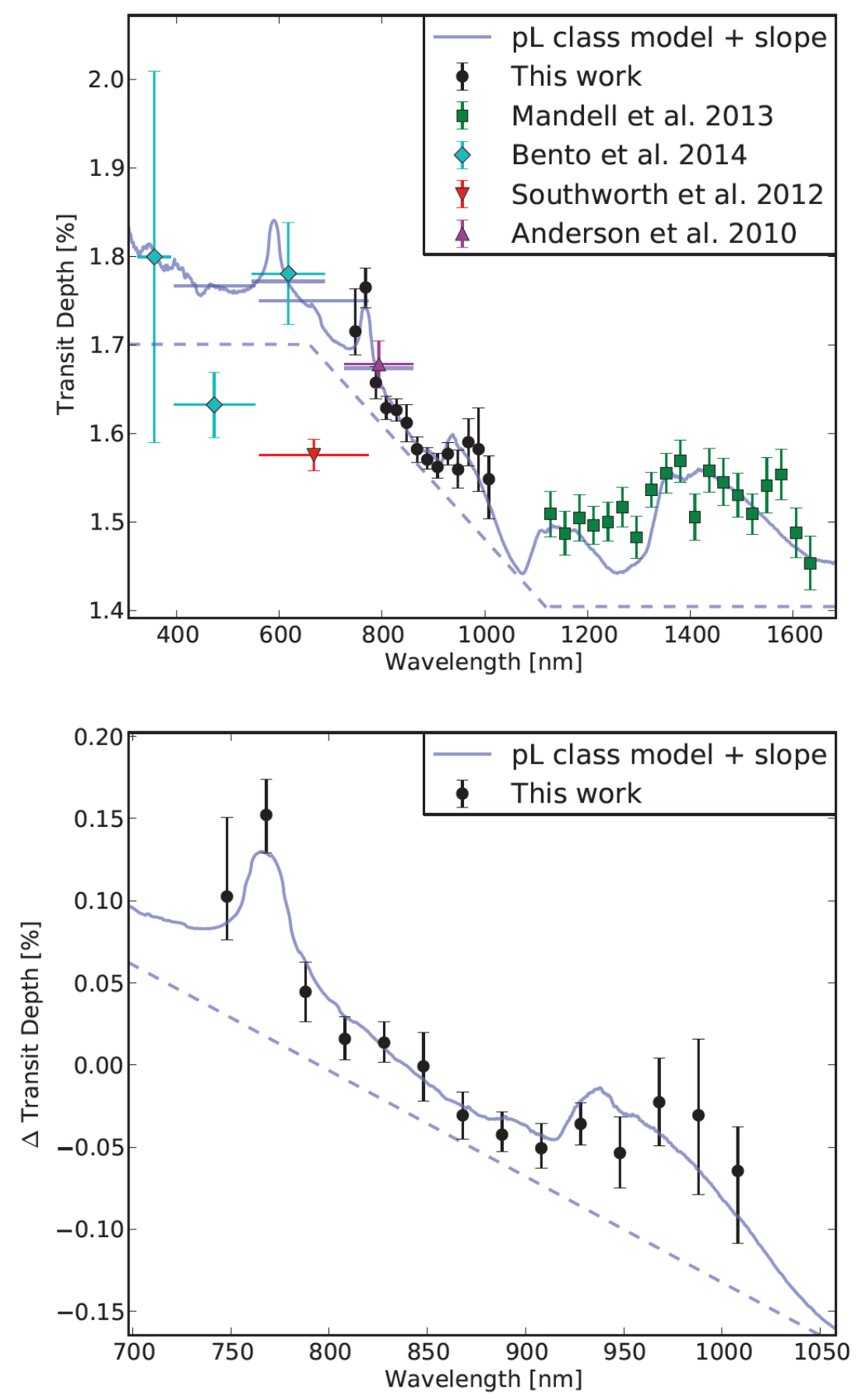

Figure 2.22: Top panel: our data points (circles) set into context with literature values of Bento et al. (2014) (diamonds), Southworth et al. (2012) (downwards arrow), Anderson et al. (2010) (upwards arrow) and Mandell et al. (2013) (squares). A baseline transit depth of $\delta_{\text {white light }}=1.612$ was assumed for our data, enforcing consistency between our data and the Anderson et al. (2010) value. A theoretical pL class like model with a slope added to the region in which we observed is shown in blue (solid line). Bottom panel: the relative transit depth variation of our data and the theoretical pL class like model with an added slope (solid blue line). 

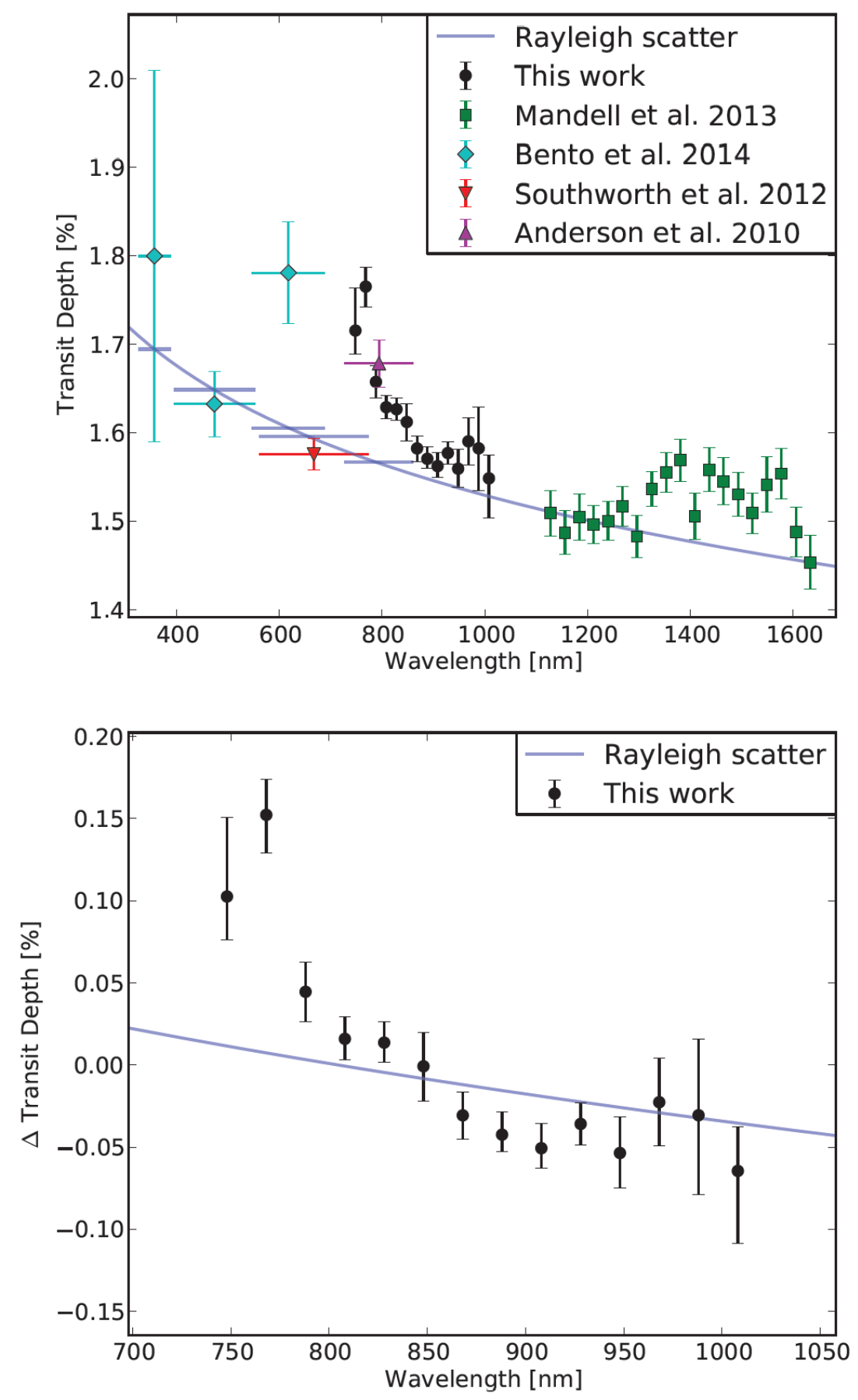

Figure 2.23: Top panel: our data points (circles) set into context with literature values of Bento et al. (2014) (diamonds), Southworth et al. (2012) (downwards arrow), Anderson et al. (2010) (upwards arrow) and Mandell et al. (2013) (squares). A baseline transit depth of $\delta_{\text {white light }}=1.612$ was assumed for our data, enforcing consistency between our data and the Anderson et al. (2010) value. A theoretical Rayleigh scattering curve is shown in blue (solid line). Bottom panel: the relative transit depth variation of our data and the theoretical Rayleigh scattering curve (solid blue line). 
depth when compared to the case of a spotless star. This effect would be stronger at shorter wavelength where the contrast between the flux emitted by the cold spot $F_{\text {spot }}$ and the flux emitted from the hotter stellar surface $F_{\star}$ is larger. To derive the true transit depth, the light curves would have to be corrected like it was done for infrared transit light curves of HD 189733b by Désert et al. (2011). A detailed overview to the math associated with this effect can be found in the paper of Ballerini et al. (2012). For our case we estimate that $90 \%$ of the surface of WASP-17b would have to be covered in star spots to cause a transit depth variation as large as the observed, rendering this explanation implausible.

\subsubsection{Third-light contamination}

In a rather similar scenario the additional flux of an unresolved optical companion could offset the light curve baseline upwards causing a smaller overall transit depth. Recent example cases for such a dilution of the transit signal by a previously unknown optical companion are the hot Jupiters WASP-12b and HAT-P-32b, both of which are, like WASP-17b, objects of large interest for the exoplanet atmosphere community (Bergfors et al. 2013; Crossfield et al. 2012; Adams et al. 2013). In the case of WASP-17b the optical companion would have to be of very low temperature, most likely an M-dwarf, causing the effect to be stronger toward longer wavelength, where the M-dwarf would be more luminous. When the impact of limb darkening is neglected the erroneous transit depth measured if a an additional light source is present, is given by Eq. 2.10, where $\kappa$ is the ratio of the 'optical companion'- to 'planet host star intensity'.

$$
\left(R_{\mathrm{p}} / R_{\star}\right)_{\text {third light }}^{2} \approx \frac{\left(R_{\mathrm{p}} / R_{\star}\right)^{2}}{(1+\kappa)}
$$

To cause the observed effect a contaminating source would have to be $5.4 \%$ as bright as the host star at $1018 \mathrm{~nm}(\Delta \mathrm{mag} \approx 3.2)$ and, therefore, would show as a significant anomaly in any red optical and infrared observations. Diffraction limited adaptive optics imaging of WASP-17 in the $K$ band as part of a larger survey searching for companions of planet host stars with the NIRC2 instrument on Keck II, however, yielded no detection of an optical companion within 5.5" (Ngo et al. 2015). We, therefore, consider the scenario of transit dilution by an optical companion to be implausible.

\subsection{Conclusion}

Our observations verify the large potential of the FORS2 instrument for transit transmission spectroscopy, which has been put forward by the works of Bean et al. from 2010 and 2011. We, however, have found that when using the instrument during large changes of the paralactic angle of the observations and under varying LADC prism distances, instrument systematics of an amplitude of $\approx 2 \%$, i.e. a similar amplitude as the expected transit signal, emerge, rendering the analysis of the data difficult. We were able to correct the large scale systematics using a 
parameterized model using the auxiliary parameters of paralactic angle and LADC prism distance. The uncertainty of our result for the absolute transit depth from this approach might be underestimated despite careful consideration of parameter correlations and red noise. The correction of the lower amplitude systematics, which finally enabled us to yield the precision necessary for the retrieval of the planetary atmospheric transmission signal, required an empirically derived noise signal. This transmission signal could be derived with high precision as it is unaffected by the uncertainty in the absolute transit depth. Our $20 \mathrm{~nm}$ wide channel light curves are of the precision of 491-1299 ppm, which is common for recent transmission spectroscopy investigations.

The resulting transmission spectrum shows a steep incline towards larger planet radii with lower wavelength, hinting at a strong absorber in the blue wavelength region. A comparison with literature values shows that this slope acts as a connection between the two spectrophotometric studies in neighboring wavelength regions by Mandell et al. (2013) and Bento et al. (2014). The amplitude of the planet radius/transit depth variation is consistent with the theory for hot atmospheres containing gaseous $\mathrm{TiO}$ and VO. However, the typical broad bands associated with these absorbers are not reproduced by our data. Instead, the spectrum is more consistent with cooler models in which potassium and water absorption dominate, if a linear slope is added to the model. There is, however, no solid physical explanation for a slope as steep as the one required.

We conclude that the tested theoretical models cannot fully explain our observations. The study of this peculiar planet would strongly benefit from further observations, especially at short wavelength where high resolution spectrophotometry could complement the existing broadband measurements. In general probing the transition regions between existing studies should be considered as a tool to eliminate the possibility of systematic transit depth offsets between the individual studies. Last but not least, the benefit that the field of exoplanet atmosphere observations could gain from the FORS2 instrument should improve drastically if instrument systematics can be avoided. Now that the LADC has been exchanged by an uncoated version, it will be possible to determine if all systematics were caused by this element or if additional sources of more subtle red noise are present. The first tests of the FORS2 performance improvement after the prism exchange by Boffin et al. (2015), Sedaghati et al. (2015) has shown promising results, and hopefully more are to come in the near future. 



\section{Chapter 3}

\section{Confirmation of the flat transmission spectrum of HAT-P-32Ab with GTC/OSIRIS \& Characterization of the M-dwarf companion HAT-P-32B}

All sections but the last section of this chapter have been published in Astrophysics \& Astronomy as the article 'The GTC exoplanet transit spectroscopy survey. IV. Confirmation of the flat transmission spectrum of HAT-P-32b' (Nortmann et al. 2016) which is reproduced in under Sect. 3.1; Credit: L. Nortmann, E. Pallé, F. Murgas, S. Dreizler, N. Iro and A. Cabrera-Lavers, A\&A, 594, A65, 2016, reproduced with permission $\mathrm{CESO}$.

The study of the transmission spectrum of the planet HAT-P-32Ab (also commonly known as HAT-P-32b) is described in the main article and the study of HAT-P-32B, the M-dwarf companion to the system, is described in the appendix of the article. The final section of this chapter, Sect. 3.2, is a conclusion of the findings regarding the M-dwarf companion HAT-P-32B and has not been previously published as part of the article.

\subsection{Paper: The GTC exoplanet transit spectroscopy survey. IV. Confirmation of the flat transmission spectrum of HAT-P-32b}




\title{
The GTC exoplanet transit spectroscopy survey
}

\section{Confirmation of the flat transmission spectrum of HAT-P-32b *}

\author{
L. Nortmann ${ }^{1}$, E. Pallé ${ }^{2,3}$, F. Murgas ${ }^{4,5}$, S. Dreizler ${ }^{1}$, N. Iro ${ }^{6}$, and A. Cabrera-Lavers ${ }^{2,3}$ \\ 1 Institut für Astrophysik, Georg-August-Universität Göttingen, Friedrich-Hund-Platz 1, 37077 Göttingen, Germany \\ e-mail: nortmann@astro.physik.uni-goettingen.de \\ 2 Instituto de Astrofísica de Canarias (IAC), 38205 La Laguna, Tenerife, Spain \\ 3 Departamento de Astrofísica, Universidad de La Laguna (ULL), 38206 La Laguna, Tenerife, Spain \\ ${ }^{4}$ Univ. Grenoble Alpes, IPAG, 38000 Grenoble, France \\ 5 CNRS, IPAG, 38000 Grenoble, France \\ 6 Theoretical Meteorology group, Klimacampus, University of Hamburg, Grindelberg 5, 20144 Hamburg, Germany
}

Received 7 September 2015 / Accepted 18 April 2016

\begin{abstract}
We observed the hot Jupiter HAT-P-32b (also known as HAT-P-32Ab) to determine its optical transmission spectrum by measuring the wavelength-dependent, planet-to-star radius ratios in the region between 518-918 nm. We used the OSIRIS instrument at the Gran Telescopio CANARIAS (GTC) in long-slit spectroscopy mode, placing HAT-P-32 and a reference star in the same slit and obtaining a time series of spectra covering two transit events. Using the best quality data set, we were able to yield 20 narrowband transit light curves, with each passband spanning a $20 \mathrm{~nm}$ wide interval. After removal of all systematic noise signals and light curve modeling, the uncertainties for the resulting radius ratios lie between 337 and $972 \mathrm{ppm}$. The radius ratios show little variation with wavelength, suggesting a high altitude cloud layer masking any atmospheric features. Alternatively, a strong depletion in alkali metals or a much smaller than expected planetary atmospheric scale height could be responsible for the lack of atmospheric features. Our result of a flat transmission spectrum is consistent with a previous ground-based study of the optical spectrum of this planet. This agreement between independent results demonstrates that ground-based measurements of exoplanet atmospheres can give reliable and reproducible results despite the fact that the data often is heavily affected by systematic noise as long as the noise source is well understood and properly corrected. We also extract an optical spectrum of the M-dwarf companion HAT-P-32B. Using PHOENIX stellar atmosphere models we determine an effective temperature of $T_{\text {eff }}=3187_{-71}^{+60} \mathrm{~K}$, which is slightly colder than previous studies relying only on broadband infrared data.
\end{abstract}

Key words. planets and satellites: atmospheres - techniques: spectroscopic

\section{Introduction}

Two decades after the first detection of an exoplanet around a solar-type star by Mayor \& Queloz (1995) the field of exoplanet science is fast moving and has expanded into many subfields. A new main focus is the characterization of exoplanet atmospheres. The most successful approach for studying the atmospheric properties of planets has been the measurement of their transmission and emission spectra from multicolor observations of the occultation events in transiting planetary systems. While the emission from a planet can be inferred from the drop in flux during the secondary eclipse, the transmission spectrum of a planet can be obtained during the primary eclipse. This is possible since the atmosphere of planets are opaque at wavelengths, where the atmospheric constituents absorb light causing larger effective planet radii and, thus, deeper transits. Many successful measurements of wavelength-dependent planet radii have been obtained from space using the Hubble Space Telescope (HST; e.g., Charbonneau et al. 2002; Pont et al. 2008; Berta et al. 2012; Sing et al. 2015). Moreover, in the last four

\footnotetext{
* The 20 narrowband and white light curves are only available at the CDS via anonymous ftp to cdsarc.u-strasbg. fr (130.79.128.5) or via

http://cdsarc.u-strasbg.fr/viz-bin/qcat?J/A+A/594/A65
}

years, ground-based observations have also yielded promising results (e.g., Bean et al. 2010; Murgas et al. 2014; Jordán et al. 2013; Gibson et al. 2013a). However, both space-based and ground-based data often are affected by systematic noise signals, which need to be addressed before a high quality transmission spectrum can be extracted. In the past, the correct treatment of these noise signals was subject of scientific debate and led to disagreements between the conclusions of several groups studying the same data sets (e.g., Tinetti et al. 2007; Ehrenreich et al. 2007; Désert et al. 2009; Gibson et al. 2011, for HD 189733b). As a general consequence, this has created doubts concerning the robustness of presented results. In this paper, we aim to demonstrate with the case of HAT-P-32b that reliable results for the transmission spectrum of a planet can be obtained from the ground. The hot Jupiter with a mass of $M=0.860 \pm 0.16 M_{\text {Jup }}$ and a radius of $R=1.789 \pm 0.025 R_{\text {Jup }}$ was discovered by Hartman et al. (2011) around a late-type F dwarf star (Vmag = 11.44 ) at an 2.15 day orbit. The dayside temperature of the planet was measured at $T_{\text {eq }}=2042 \pm 50 \mathrm{~K}$ by Zhao et al. (2014) from secondary eclipse observations in the $H, K_{\mathrm{S}}, 3.6$, and $4.5 \mu \mathrm{m}$ bands. An optically close companion was discovered in 2013 by Adams et al. The stellar companion was recently studied in more detail and concluded to be an M-dwarf bound to the HAT-P-32 system from proper motion and AO measurements 
(Ngo et al. 2015; Zhao et al. 2014). Both studies place the effective temperature of the companion at about $T_{\text {eff }} \approx 3500 \mathrm{~K}$. Following the notation used in these works, in the following we refer to this stellar companion as HAT-P-32B and to the planet host star and the planet as HAT-P-32A and HAT-P-32Ab, respectively. Knutson et al. (2014) observed the HAT-P-32 system among several other planet host stars for radial velocity (RV) trends that could indicate additional companions. They found a long trend signal for HAT-P-32A pointing to the existence of yet another body in the system. A transit timing variation (TTV) study of 45 transit events by Seeliger et al. (2014) looking for evidence of an additional body found no evidence for variations larger than $1.5 \mathrm{~min}$.

Gibson et al. (2013b) obtained a ground-based optical transmission spectrum of HAT-P-32Ab using the Gemini MultiObject Spectrograph (GMOS; Davies et al. 1997) at Gemini North. Their results show a flat transmission spectrum. We probed a very similar wavelength range using the long-slit method at the OSIRIS instrument at the 10-meter class telescope Gran Telescopio CANARIAS (GTC) aiming to verify the nature of the transmission spectrum and further demonstrate the potential of GTC/OSIRIS as a reliable survey instrument for observations of this kind.

The paper is organized as follows. We first describe the observing setup and data reduction in Sect. 2. This is followed by a description of the white light curve analysis in Sect. 3 and a discussion of the white light curve results in Sect. 4. Here we also address systematic noise signals we found in both data sets. In Sect. 5 we describe the source of the largest noise signal and motivate its correction for the narrowband light curves followed by a description of the extraction of the transmission spectrum during the analysis of the narrowband light curves in Sect 6 . We present and discuss our results for the transmission spectrum in Sect. 7 and draw our conclusions in Sect. 8. The study of photometric and spectroscopic data of the companion HAT-P-32B to derive its stellar parameters and extract values necessary for the correction of its diluting effect on the transit depth of HAT-P-32Ab can be found in Appendix A.

\section{Observations and data}

We observed HAT-P-32Ab twice during transit on 2012 September 15 (JD 2456185.5 , hereafter referred to as Run 1) and on 2012 September 30 (JD 2456200.5 , hereafter referred to as Run 2) with the OSIRIS instrument (Optical System for Imaging and low-Intermediate-Resolution Integrated Spectroscopy; Sánchez et al. 2012) mounted at the Spanish $10.4 \mathrm{~m}$ telescope GTC. We chose the method of long-slit spectroscopy, in which the planet host star and a suitable reference star are both placed inside one long slit. The grism R1000R was used to disperse the light over the range from 518 to $918 \mathrm{~nm}$. With an exposure time of $10 \mathrm{~s}$ (Run 1) and $7 \mathrm{~s}$ (Run 2) continuous time series of 321 (Run 1) and 700 (Run 2) optical spectra were obtained each night covering the duration of the whole transit event in both cases. We used slightly different observing setups each night, working with different regions of the CCD detector. The OSIRIS detector consists of a mosaic of two $2048 \times 4096$ pixel Marconi CCD42-82 chips. In Run 1 the chosen reference star Ref1 was considerably fainter $(\Delta V \operatorname{mag}=2.15)$ than HAT-P-32A and located at a distance of $56.0^{\prime \prime}=0.93^{\prime}$. A custom made $12^{\prime \prime}$ wide slit was used and both stars were placed on CCD1. In Run 2 we chose a brighter reference star, $\operatorname{Ref} 2,(\Delta V \operatorname{mag}=-0.467)$ located at a $191.0^{\prime \prime}=3.18^{\prime}$ separation from HAT-P-32A. As a result of the larger distance between the stars, they could not both be

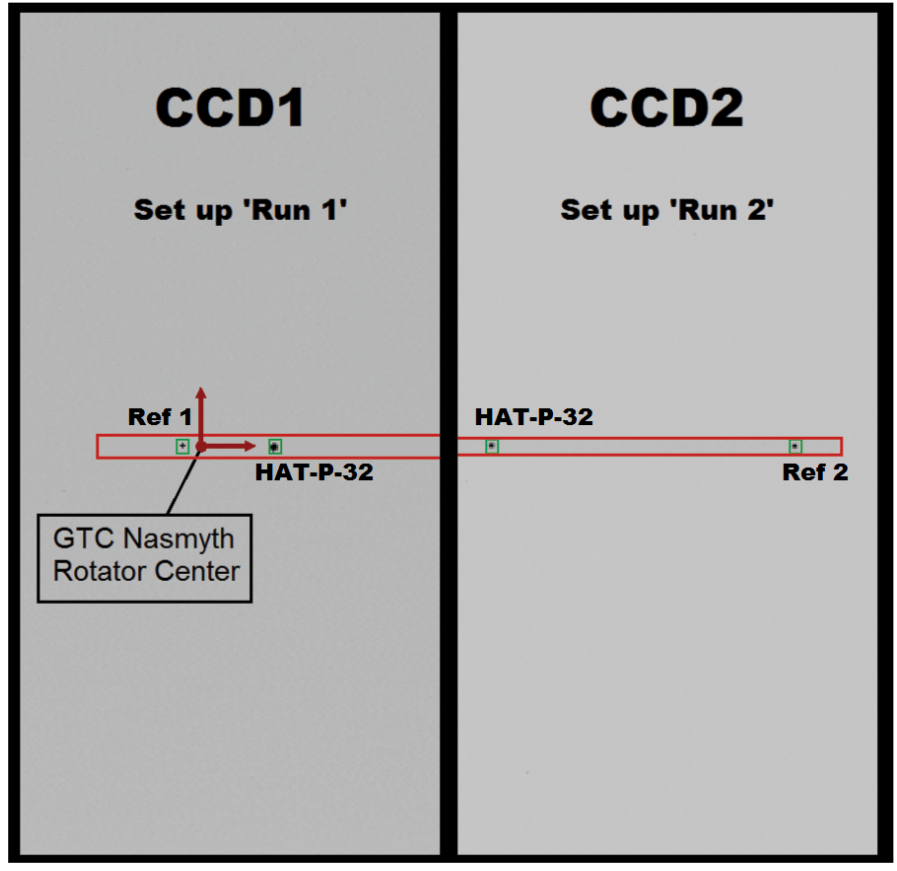

Fig. 1. Observing setup for Run 1 and Run 2. In Run 1 both stars were placed in CCD1 and a $12^{\prime \prime}$ wide slit was used. In Run 2 we placed both stars in CCD2 and used a slit of $10^{\prime \prime}$ width.

Table 1. Coordinates of the planet host star HAT-P-32A and the reference stars Ref1 and Ref2 used in the first and second observing run.

\begin{tabular}{cccc}
\hline \hline Star & RA & Dec & $V_{\text {mag }}$ \\
\hline HAT-P-32 & $02 \mathrm{~h} 04 \mathrm{~m} 10.278 \mathrm{~s}$ & $+46^{\circ} 41^{\prime} 16.21^{\prime \prime}$ & 11.44 \\
Ref1 (Run 1) & $02 \mathrm{~h} 04 \mathrm{~m} 15.060 \mathrm{~s}$ & $+46^{\circ} 40^{\prime} 49.57^{\prime \prime}$ & 13.59 \\
Ref2 (Run 2) & $02 \mathrm{~h} 03 \mathrm{~m} 51.771 \mathrm{~s}$ & $+46^{\circ} 41^{\prime} 32.23^{\prime \prime}$ & 10.97 \\
\hline
\end{tabular}

placed in CCD1. In order to have both stars on the same CCD and, thus, avoid possible complications from variations in the detector properties we placed both stars on CCD2, which has a larger unvignetted field of view through the slit than CCD1. We also exchanged the custom made $12^{\prime \prime}$ wide slit for a $10^{\prime \prime}$ wide slit, since the latter is slightly longer (extending $0.567^{\prime}$ further into CCD2) giving both stars more room in spatial direction. The setup for both observing runs is illustrated in Fig. 1 and the coordinates for both reference stars are given in Table 1 . The observing conditions during both nights were good with an average seeing of $1.06^{\prime \prime}$ in Run 1 and $1.12^{\prime \prime}$ in Run 2. The seeing was not stable in either run, varying between $0.78^{\prime \prime}$ and $1.82^{\prime \prime}$ during the first and between $0.69^{\prime \prime}$ and $2.42^{\prime \prime}$ during the second night.

Owing to complications during the observation almost no out-of-transit data was obtained during Run 1 and part of the data was rendered useless by a light reflection passing over the detector contaminating the red part of the spectrum of HAT-P32A for approximately $20 \mathrm{~min}$ (35 frames) during the second half of the transit. An example of a contaminated frame is shown in Fig. 2. After each run about 50 bias frames and 100 sky flats were taken. For wavelength calibration, spectra of the $\mathrm{HgAr}, \mathrm{Xe}$, and Ne lamps were taken with a $1^{\prime \prime}$ wide slit. 


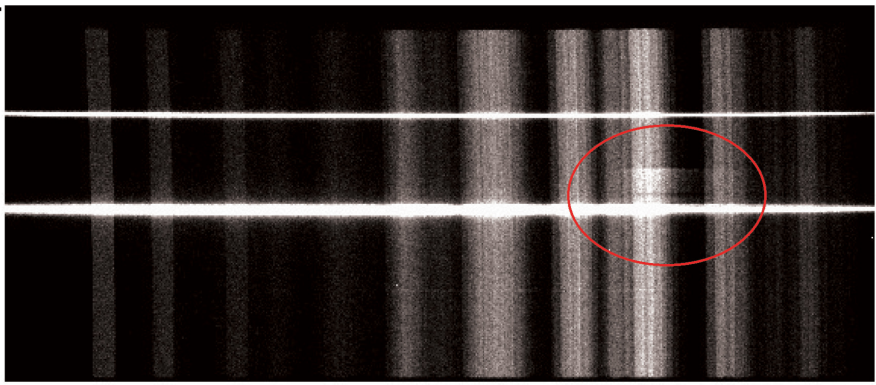

Fig. 2. Example of an image obtained in Run 1, which was contaminated by an internal light reflection. The image shows the light of HAT-P32A (bottom) and the fainter reference star Ref1 (top) dispersed by the grism in the horizontal direction. The contaminating light reflection is indicated with a red circle.

\subsection{Data reduction and spectral extraction}

We employed standard data reduction procedures, subtracting the median averaged bias and dividing by the median averaged flat field. We then applied a wavelength calibration to every column of the image rebinning the data to a homogeneous wavelength grid using the IDL routine rebinw from the PINTofALE package (Kashyap \& Drake 2000), guaranteeing flux conservation. This step ensured that every pixel in an image row corresponded to the same wavelength. The extraction of the stellar spectra from the images was then performed using the optimal extraction algorithm (Horne 1986). The algorithm performed well on our data sets, yielding lower noise levels than other, more simple approaches. During the extraction a median averaged spatial profile was used to identify and mask cosmic ray strikes. Due to the close projected distance of HAT-P-32A and the fainter M-dwarf companion (which was not yet discovered at the time of the observations) it was not possible to reliably exclude HAT-P-32B by choosing a narrow extraction aperture. Instead, we choose a very wide aperture $\left(80\right.$ pixel $\left.=20.32^{\prime \prime}\right)$ ensuring that HAT-P-32B was fully within this aperture at all times. As a consequence, the diluting effect of its additional flux on the transit depth had to be corrected during transit modeling (see Sect. 3).

During the observations the stars drifted slightly in spatial and also in dispersion direction. We monitored the drift in spatial direction by fitting a Gaussian function to the stellar profile tracing the position of the peaks. In this step, we also retrieved the full width at half maximum (FWHM) of the fitted Gaussian profile to monitor the seeing variations. The drift of the stars in dispersion direction caused small shifts in the wavelength solution with respect to that obtained for a star perfectly centered within the slit. We monitored and corrected these wavelength shifts by calculating the cross correlation of each spectrum with the first spectrum of the respective run.

\subsection{Light curves}

Since any telluric variations during the observing runs will have affected both planet host star and reference star in the same manner, these effects can be neglected if only the relative light curves of these two objects are considered. We created white light curves from the data by dividing the total summed flux over all wavelengths for HAT-P-32A by the total sum of the reference star spectra for each measurement. The resulting white light curves are shown in Fig. 3. We further created 20 narrowband channel light curves from the data of Run 2 by dividing
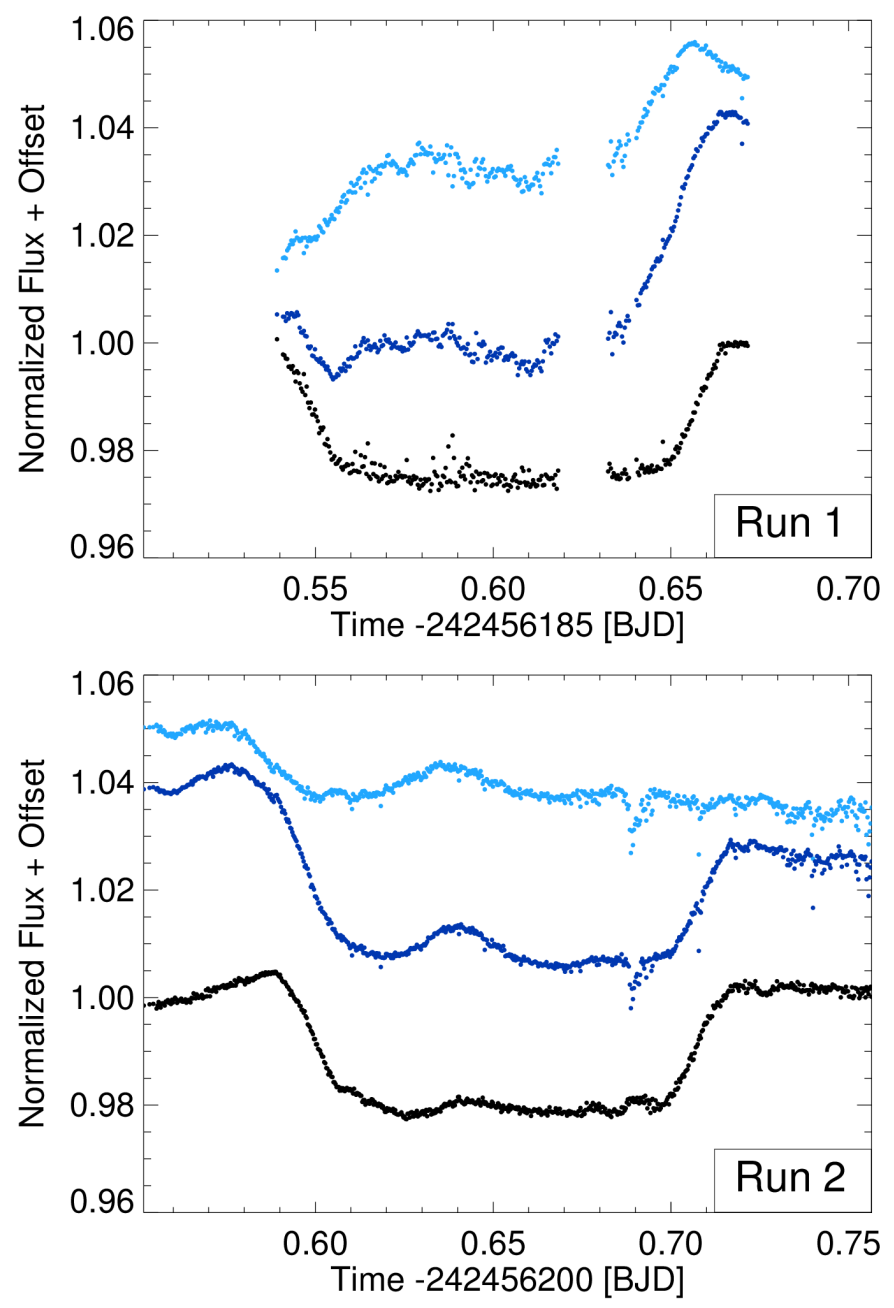

Fig. 3. Raw white light curves for HAT-P-32A (dark gray circles) and the reference star (light gray circles) for both observing runs and their division the relative white light curve (black circles). Top panel: data for Run 1, bottom panel: data for Run 2.

the wavelength range into intervals of $20 \mathrm{~nm}$. The spectra of HAT-P-32A and reference star Ref2 are shown in Fig. 4 together with the channel limits. Channel \#13 encompassed both the telluric oxygen bands and the potassium resonance lines (K I at 766.5 and $769.9 \mathrm{~nm}$ ) predicted for exoplanet atmospheres at moderate temperatures. Akin to what has been reported by Parviainen et al. (2016), we found that the noise level, estimated from the standard deviation of the out-of-transit light curve scatter in each individual wavelength point, rises significantly in the deeper of the two telluric oxygen bands, negatively affecting the signal-to-noise of the entire channel \#13 light curve. We therefore constructed an additional channel (channel \#13b) of only $15 \mathrm{~nm}$ width, spanning from 763 to $788 \mathrm{~nm}$. This channel was still encompassing the expected potassium lines but avoided the high noise region as sketched in Fig. 5.

\section{Analysis of white light curves}

The relative white light curves for both runs are shown in Fig. 3. We found both white light curves to be affected by red noise. Previous works dealing with data obtained with GTC/OSIRIS have reported on similar noise signals (Sing et al. 2012; Murgas et al. 2014; Mallonn et al. 2015). The three works explored different systematics models that included terms depending on the seeing 


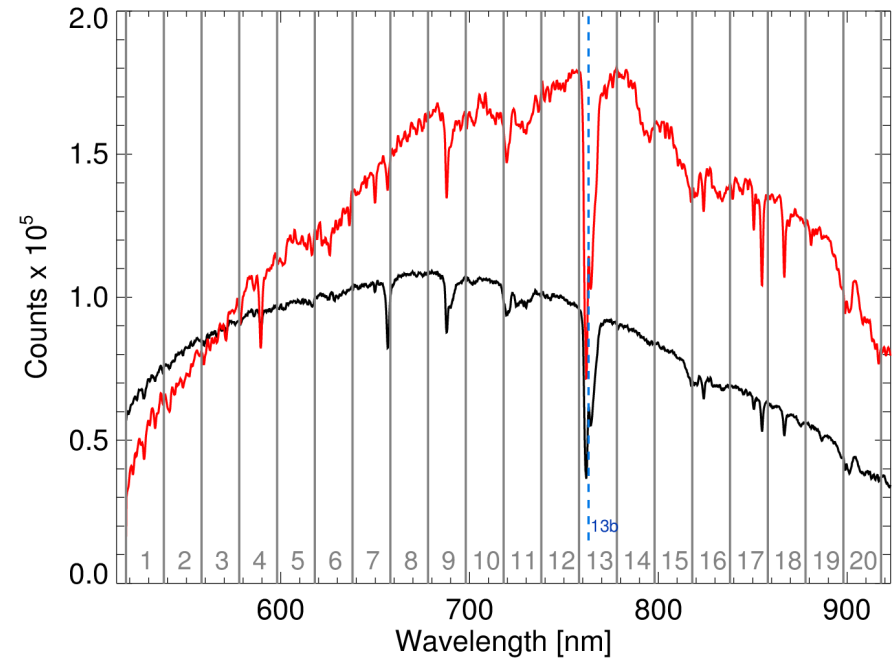

Fig. 4. Spectra of HAT-P-32A and reference star Ref2 from Run 2. Indicated in gray are the limits of the twenty $20 \mathrm{~nm}$ wide narrowband channels. The blue limit of the additionally defined $15 \mathrm{~nm}$ wide channel $\# 13 \mathrm{~b}$ excluding the first $5 \mathrm{~nm}$ of channel \#13 is indicated with a blue dashed line.
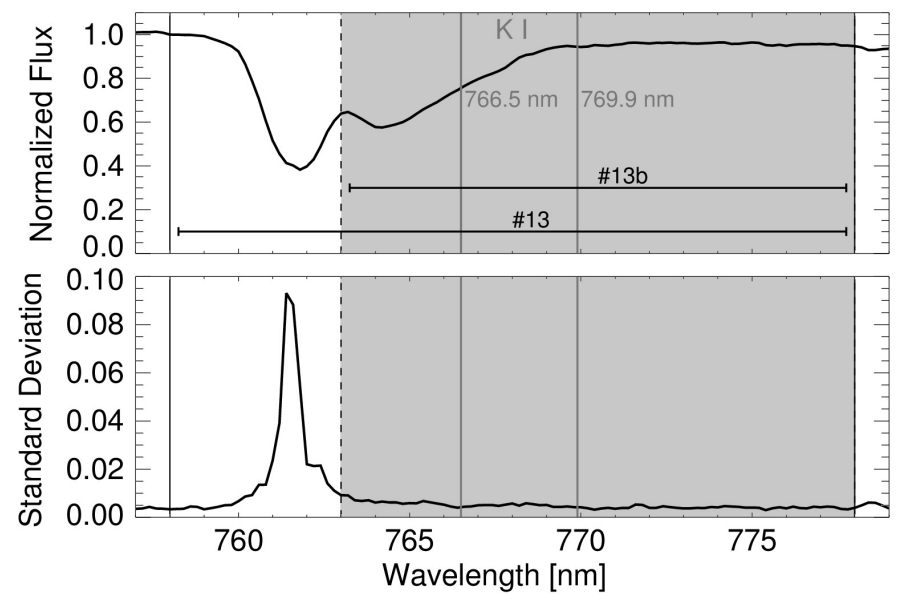

Fig. 5. Definition of narrowband channel \#13b as a subchannel of channel \#13. The gray shaded area encompasses the predicted K I resonance lines but avoids the high noise region caused by telluric oxygen absorption. Top panel: example spectrum of Ref2 within the limits of narrowband channel \#13, showing the significant flux decrease in the telluric oxygen. Bottom panel: noise level at each wavelength as estimated from the after transit light curve standard deviation showing a strong increase of noise in the stronger telluric oxygen absorption band.

and air mass. We also found indicators for a possible correlation of the red noise with auxiliary parameters and explored these possible correlations by including a systematic noise model in our light curve fitting. We found the minimal $\chi^{2}$ using the IDL implementation of the Levenberg-Markward algorithm mpfit by Markwardt (2009), while modeling the light curves with a model of the form

$\mathcal{M}=\left(\mathcal{T}+c_{w}\right) \cdot \mathcal{S}$,

where $\mathcal{T}$ is the analytical transit model described by Mandel \& Agol (2002), $\mathcal{S}$ is a systematic noise model and $c_{w}$ is the relative flux contribution of the stellar companion HAT-P$32 \mathrm{~B}$ in white light (i.e., $c_{w}=f_{\text {HAT-P-32B }} / f_{\text {HAT-P-32A }}$, where $f$ is the total flux integrated over the white light wavelength range). In the following we refer to $c_{w}$ and its equivalents for the narrowband channels as the dilution factor. The Mandel \& Agol transit model parameterizes the transit using the radius ratio between the planet and star $R_{\mathrm{p}} / R_{\star}$, the semimajor axis of the planet orbit in units of the stellar radius $a_{\mathrm{p}} / R_{\star}$, the inclination of the planet orbit $i$, a reference time for the midtransit $T_{\mathrm{C}_{1}}$ and two coefficients, $u_{1}$ and $u_{2}$, describing the stellar limb darkening with a quadratic limb darkening law. Also needed is the period of the planet orbit $P$, which we kept fixed to the value given by Hartman et al. (2011), i.e., $P=2.150008$ days, and the eccentricity of the orbit $e$, which we kept fixed at $e=0$. The code implementation of Eastman et al. (2013) was used for the calculation of the transit model. The relative flux contribution of the stellar M-dwarf companion $c_{w}$ and its uncertainty were determined from our own data of Run 2, as described in detail in Appendix A, and this value was kept fixed during this optimization step. We tested 135 different systematic noise models $\mathcal{S}$, where each model was a different combination of polynomial functions depending on the four auxiliary parameters: position in spatial direction xpos, position in dispersion direction ypos, seeing/FWHM of the stellar profile fwhm, and air mass airm. All combinations of different polynomial orders between 0 and 2 (between 0 and 4 for the FWHM) for all four auxiliary parameters were explored. The most complex form of $\mathcal{S}$ we tested, consequently, was of the form

$$
\begin{aligned}
\mathcal{S}= & n_{0}+x_{1} \cdot \text { xpos }+x_{2} \cdot \text { xpos }^{2} \\
& +y_{1} \cdot \text { ypos }+y_{2} \cdot \text { ypos }^{2} \\
& +a_{1} \cdot \text { airm }+a_{2} \cdot \text { airm }^{2} \\
& +f_{1} \cdot f w h m+f_{2} \cdot f w h m^{2}+f_{3} \cdot f w h m^{3}+f_{4} \cdot f w h m^{4},
\end{aligned}
$$

where $n_{0}$ is the normalization and $x_{1,2}, y_{1,2}, a_{1,2}$ and $f_{1,2,3,4}$ are model parameters scaling the influence of the respective auxiliary parameter.

To avoid overfitting because the minimal $\chi^{2}$ generally decreases with a rising number of free parameters, a compromise between the number of free parameters and minimization of the $\chi^{2}$ should be found by means of an objective criterion. We used the Bayesian information criterion for the case of an unknown variance of the data

$\mathrm{BIC}=N \ln (R S S / N)+k \cdot \ln (N)$,

where $R S S$ is the sum of squared residuals, $k$ is the number of free parameters, and $N$ is the number of data points, to determine the best choice of $\mathcal{S}$. We compared the BIC of all tested models and found that using a combination of a first-order polynomial of the position drift in dispersion direction (ypos), a first-order polynomial of the air mass, and a third-order polynomial function of the FWHM yields the lowest BIC for the data of Run 1, i.e., a model of the form

$$
\begin{aligned}
\mathcal{S}_{\text {Run } 1}^{*}= & n_{0}+y_{1} \cdot \text { ypos }+a_{1} \cdot \text { airm } \\
& +f_{1} \cdot f w h m+f_{2} \cdot f w h m^{2}+f_{3} \cdot f w h m^{3}
\end{aligned}
$$

An abbreviated compilation of the results of the BIC comparison can be found in Table 2, where, for practicality, only the models with an $\triangle \mathrm{BIC}=\mathrm{BIC}-\mathrm{BIC}_{\min }<10$ are listed. The bestfit results for the planet-to-star radius ratio derived using these model approaches differ only slightly, and all lie well within the $1 \sigma$ uncertainty interval (derived in Sect. 3.1) of the best-fit result obtained with the lowest BIC model. In Table 2 the models are identified by a short code, which is to be read as follows: "xpos" is the key word for the position drift in spatial direction, "ypos" for the drift in dispersion direction, "airm" for the air mass, and "fwhm" for the seeing/FWHM of the stellar profile. The number 
Table 2. Model comparison for the white light curve of Run 1.

\begin{tabular}{ccc}
\hline \hline Model & $\Delta \mathrm{BIC}$ & $\Delta R_{\mathrm{p}} / R_{\star}$ \\
\hline xpos0 ypos0 airm0 fwhm3 & 5.76 & 0.000586 \\
xpos0 ypos0 airm1 fwhm3 & 4.28 & 0.000292 \\
xpos0 ypos0 airm1 fwhm4 & 9.63 & 0.000332 \\
xpos0 ypos1 airm0 fwhm3 & 7.96 & 0.000399 \\
xpos0 ypos1 airm1 fwhm2 & 6.92 & 0.000050 \\
xpos0 ypos1 airm1 fwhm3 & $\mathbf{0 . 0 0}$ & 0.000000 \\
xpos0 ypos1 airm1 fwhm4 & 5.70 & -0.000125 \\
xpos0 ypos2 airm1 fwhm3 & 4.63 & 0.000019 \\
xpos1 ypos0 airm0 fwhm3 & 7.23 & 0.000300 \\
xpos1 ypos0 airm1 fwhm3 & 9.77 & 0.000443 \\
xpos1 ypos0 airm2 fwhm3 & 7.46 & 0.000492 \\
xpos1 ypos1 airm0 fwhm3 & 5.54 & -0.000456 \\
xpos1 ypos1 airm1 fwhm3 & 5.69 & 0.000046 \\
xpos1 ypos2 airm0 fwhm3 & 9.77 & -0.000346 \\
\hline
\end{tabular}

Notes. Different model approaches for the systematic noise and the respective values for $\triangle \mathrm{BIC}=\mathrm{BIC}-\mathrm{BIC}_{\min }$ and the respective change in best-fitting planet-to-star radius ratio $\Delta R_{\mathrm{p}} / R_{\star}=R_{\mathrm{p}} / R_{\star}-\left(R_{\mathrm{p}} / R_{\star}\right)_{\mathrm{BIC}_{\min }}$.

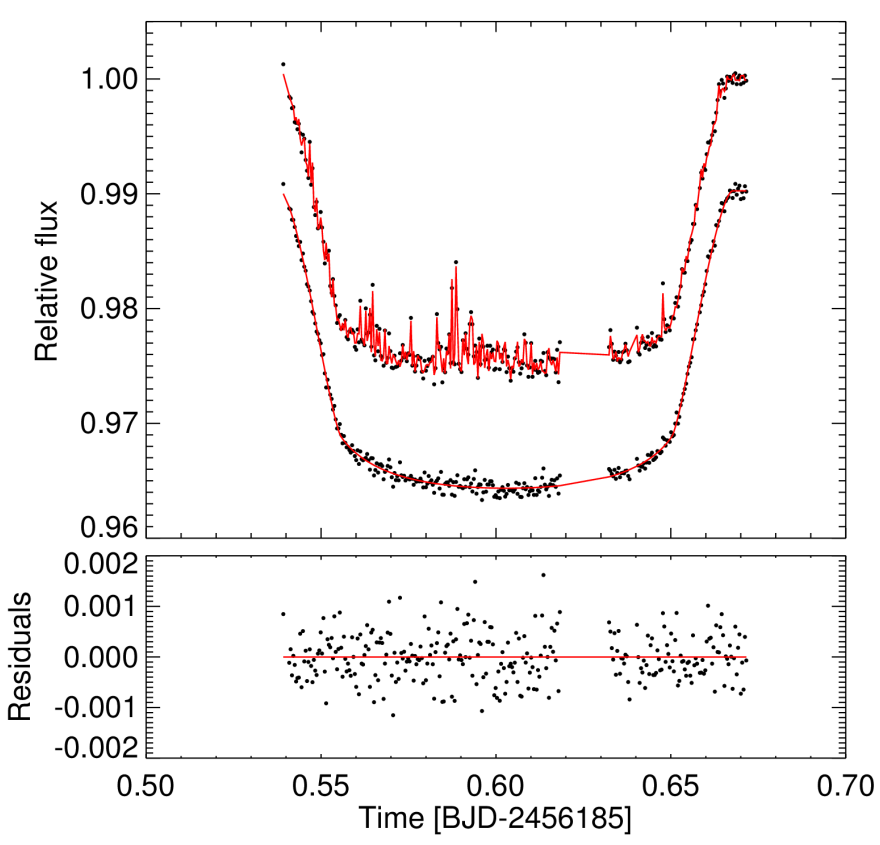

Fig. 6. Top panel: white light curve from Run 1 (black points) with bestfit model (red line) before (top) and after (bottom) division by the bestfit systematic noise model (see Sect. 3). The bottom curve was shifted downward by a small offset for clarity. Bottom panel: residuals of the white light curve from Run 1 after subtraction of the best-fit model.

following each of these key words indicates the highest polynomial order that was allowed to be non-zero and free in the model fit. Using the systematic noise model $\mathcal{S}_{\text {Run } 1}^{*}$ given in Eq. (4) we achieved a good fit to the data of Run 1 with an almost Gaussian distribution of the residuals (with a normalized standard deviation of $463 \mathrm{ppm}$ ). The remaining correlation of the residuals is explored in the Sect. 3.1, which focuses on the error estimates of the results. A plot of the white light curve with the best-fit model can be found in Fig. 6. After repeating this process for the data of Run 2, we found the minimal BIC for a systematics model consisting of a first-order polynomial of the position drift in spatial direction (xpos), a first-order polynomial of the

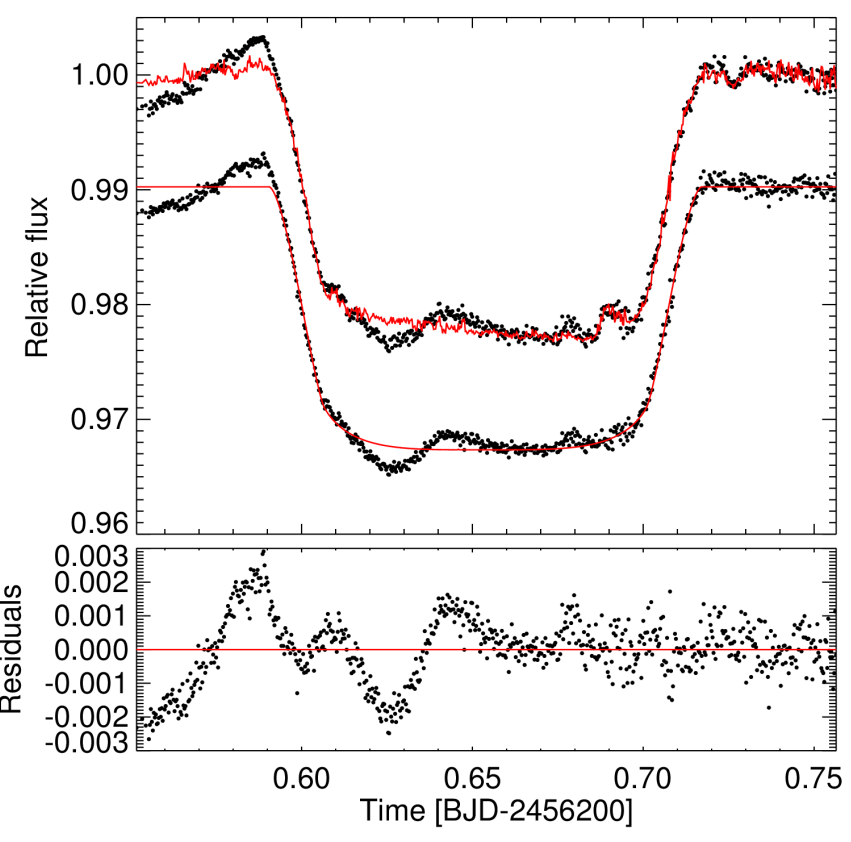

Fig. 7. Top panel: white light curve from Run 2 (black points) with bestfit model (red line) before (top) and after (bottom) division by the bestfit systematic noise model (see Sect. 3). The bottom curve was shifted downward by a small offset for clarity. Bottom panel: residuals of the white light curve from Run 2 after subtracting the best-fit model.

air mass, and a third-order polynomial function of the FWHM. However, neither this model nor any other tested combination yielded a good fit to the whole curve leaving the residuals still strongly correlated (see Fig. 7). This lead us to conclude that the systematic noise, which is strongly distorting the first part of the light curve of Run 2, has a different origin. We investigate this origin in Sect. 5.

\subsection{Errors}

For the white light curve fit of Run 1 we probe the probability distributions of the model parameters with a Markov chain Monte Carlo (MCMC) sampling using the emcee package (Foreman-Mackey et al. 2013). We used noninformative priors for all parameters except the dilution coefficient $c_{w}$, which we allowed to vary within its uncertainties using a Gaussian prior. The MCMC sampling was run with an ensemble of 600 walkers each starting with slightly different parameter populations as seeds for the chains. We let the chains run for 20000 accepted steps and defined a burn-in phase, in which the chains are not yet fully converged, to be over after 5000 steps omitting all chain-steps prior to this mark. The results can be considered robust if all the chains converge to the same probability distribution, which they did in our case. We determined a thinning factor for each chain as the largest autocorrelation length of any parameter within the chain. The average thinning factor was $\approx 200$, which resulted in a total of 45891 accepted sample points for the merged distribution of an accepted chain steps. The correlation plots of the posterior distributions for all parameters of the white light curve fit of Run 1 are shown in Fig. 8. The $1 \sigma$ uncertainties of each parameter were calculated as the limits encompassing $68.27 \%$ of all sampled points. 


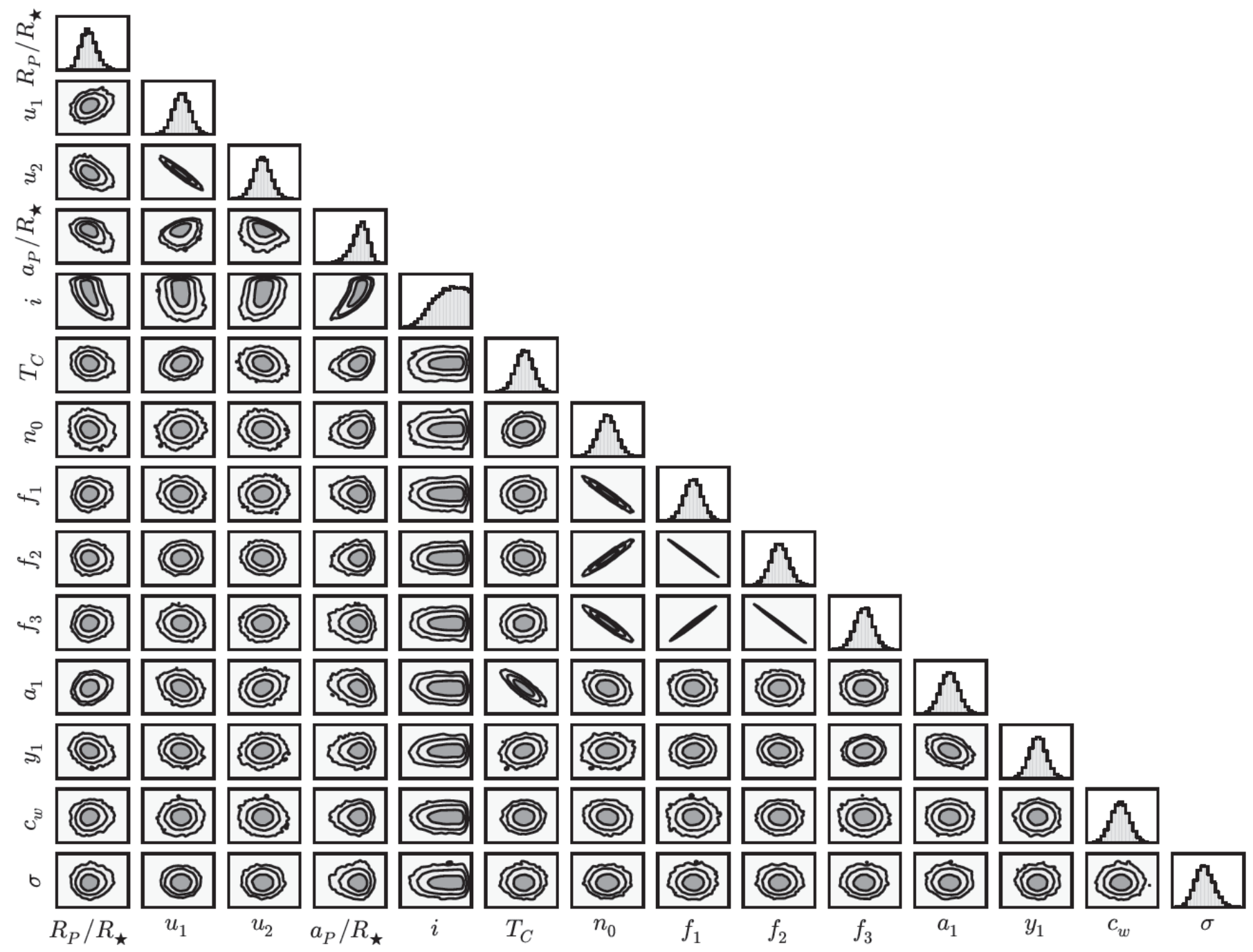

Fig. 8. Correlation plots of the posterior parameter distributions for the white light curve of Run 1 from MCMC. The lines indicate the areas encompassing $1 \sigma, 2 \sigma$, and $3 \sigma$ of the whole distribution, respectively.

\subsection{Red noise estimation}

We calculated the red noise factor $\beta$ to determine the level of red noise remaining in the light curve residuals and consequent underestimation of the parameter uncertainties. This factor was introduced by Winn et al. (2008) and is based on a comparison of the progression of the standard deviation of the time-binned light curve residuals with the behavior expected for a light curve purely affected by Gaussian (i.e., white) noise. The latter is described by Eq. (5),

$\sigma_{N}^{\text {theory }}=\frac{\sigma_{1}}{\sqrt{N}} \sqrt{\frac{M}{M-1}}$,

where $\sigma_{1}$ is the amplitude of the Gaussian white noise, $N$ the number of adjacent points binned together, and $M$ the total number of bins. The difference between theoretical and actual progression of the standard deviation of the binned residuals is caused by red noise. Winn et al. define the ratio between the two curves as the "red noise factor" $\beta=\sigma_{N}^{\text {actual }} / \sigma_{N}^{\text {theory }}$, which can be used to inflate the MCMC chain derived error bars of all parameters. We determined the maximal value of this ratio to be $\beta_{\text {Run } 1}=1.345$ for the white light curve data from the first run when the systematics model with the lowest BIC was used and $\beta_{\text {Run } 2} \geq 6.5$ for the data from Run 2, regardless of which systematics model $\mathcal{S}$ was used. The $\beta$ factor close to unity for Run 1 demonstrates that the systematics model $\mathcal{S}_{\text {Run 1 }}^{*}$ (Eq. (4)) used was appropriate to correct all noise sources. In contrast, the high value derived for Run 2 clearly indicates a remaining noise source (see Sect. 5), suggesting the model approach used for Run 1 to be insufficient for the analysis of the data of Run 2.

\section{Results and discussion of the white light curves}

The results for the white light curve fit to the data from Run 1 are listed in Table 3 together with the literature parameters of recent high precision studies of HAT-P-32Ab. The error bars of these results were inflated with the red noise factor of $\beta=1.345$ derived in Sect. 3.1. We find our results for the planet parameters to be consistent with the literature values. The difference in radius ratio compared to the results of Hartman et al. (2011) and Seeliger et al. (2014) might be explained by the different wavelength region probed in this study but is more likely caused by the transit depth dilution from additional flux of the M-dwarf companion HAT-P-32B, which was not accounted for in these two studies. 
Table 3. Best-fit planet system parameters from $\chi^{2}$ optimization and confidence intervals from MCMC sampling of the posterior parameter distributions for the Run 1 white light curve of HAT-P-32Ab.

\begin{tabular}{|c|c|c|c|c|}
\hline $\begin{array}{l}\text { Parameter } \\
\text { filter/range }\end{array}$ & $\begin{array}{c}\text { This work } \\
518-918 \mathrm{~nm}\end{array}$ & $\begin{array}{c}\text { Hartman et al. }(2011, e \equiv 0) \\
\qquad I, z, g\end{array}$ & $\begin{array}{l}\text { Gibson et al. }(2013 b) \\
520.6-932.5 \mathrm{~nm}\end{array}$ & $\begin{array}{l}\text { Seeliger et al. (2014) } \\
\quad r_{\mathrm{S}}, R_{\mathrm{B}}, R_{\mathrm{C}}, \text { Clear }\end{array}$ \\
\hline$R_{\mathrm{p}} / R_{\star}$ & $0.1516_{-0.0005}^{+0.0009}$ & $0.1508_{-0.0004}^{+0.0004}$ & $0.1515_{-0.0012}^{+0.0012}$ & $0.1510_{-0.0004}^{+0.0004}$ \\
\hline$a_{\mathrm{p}} / R_{\star}$ & $6.123_{-0.054}^{+0.021}$ & $6.05_{-0.04}^{+0.03}$ & $6.091_{-0.047}^{+0.036}$ & $6.056_{-0.009}^{+0.009}$ \\
\hline$i$ & $89.33_{-0.80}^{+0.58}$ & $88.9_{-0.4}^{+0.4}$ & $89.12_{0.68}^{+0.61}$ & $88.92_{-0.10}^{+0.10}$ \\
\hline$P[\mathrm{~d}]$ & 2.1500080 (fixed) & $2.1500080(1)$ & 2.150008 (fixed) & $2.15000825(12)$ \\
\hline$T_{\mathrm{C}_{1}}-2456185\left[\mathrm{BJD}_{\mathrm{UTC}}\right]$ & $0.602987(110)$ & $0.60294(918)^{a}$ & $0.60337(19)^{a}$ & $0.60247(19)^{a}$ \\
\hline$e$ & 0.0 (fixed) & 0.0 (fixed) & 0.0 (fixed) & 0.0 (fixed) \\
\hline$u_{1}$ & $0.1993_{-0.0430}^{+0.0420}$ & $I$ band $=0.2045$ & $0.279_{-0.074}^{+0.070}$ & $R$ band $=0.28$ \\
\hline$u_{2}$ & $0.2862_{-0.0974}^{+0.0928}$ & $I$ band $=0.3593$ & $0.254_{-0.122}^{+0.123}$ & $R$ band $=0.35$ \\
\hline$T_{\mathrm{C}_{2}}-2456200\left[\mathrm{BJD}_{\mathrm{UTC}}\right]^{a}$ & $0.65304(11)$ & $0.65299(90)$ & $0.65343(19)$ & $0.65253(19)$ \\
\hline
\end{tabular}

Notes. The red noise factor used to inflate the error bars of our results is $\beta=1.345$. For comparison the planet parameters obtained from three recent works (Hartman et al. 2011, $e \equiv 0$; Gibson et al. 2013b; Seeliger et al. 2014) are listed. ${ }^{(a)}$ Value derived from the ephemeris information given in respective papers.

A rough dilution correction of the given radius ratios using

$\left(R_{\mathrm{p}} / R_{\star}\right)_{\text {corrected }}=\left(R_{\mathrm{p}} / R_{\star}\right)_{\text {uncorrected }} \cdot \sqrt{1+c_{\text {filter }}}$,

with the dilution factor values $c_{i}^{\prime}=0.006 \pm 0.002, c_{z}^{\prime}=0.012 \pm$ $0.004, c_{g}^{\prime}<0.0018$ provided by Zhao et al. (2014) in similar broadband filters as those used in the two studies mentioned above, yields comparable radius ratios to that derived by us and Gibson et al. (2013b).

\subsection{Transit timing comparison}

In order to compare our best-fit transit time, $T_{\mathrm{C}_{1}, 2456185}$ with literature predictions, we use the ephemeris given in each paper to calculate the expected transit time and its uncertainty using Eq. (7) as follows:

$T_{\mathrm{C}}=T_{0}+E \cdot P$,

where $T_{0}$ is a reference transit time, $E$ the epoch, and $P$ the orbital period given in the respective paper. We find that our result for the transit time best agrees with the prediction given by the discovery paper (Hartman et al. 2011). Using their ephemeris information and our measured transit time to refine the period we yield $P_{\text {new }}=2.15000806(24)$ days.

We also calculated the predicted transit time for Run 2 $T_{\mathrm{C}_{2}, 2456200}$, which we could not reliably measure from a white light curve transit fit. To calculate the prediction for $T_{\mathrm{C}_{2}, 2456200}$ from our Run 1 results, we used Eq. (7) with $T_{0}=T_{\mathrm{C}_{1}, 2456185}$, $E=7$ and $P=P_{\text {new }}$. The results are given in Table 3 .

\subsection{Comparison with theoretical limb darkening}

As the limb darkening coefficients are wavelength dependent and cannot be directly compared with literature results obtained in different filters, we compared these coefficients to theoretical values. To derive these theoretical coefficients, we calculated the wavelength-dependent theoretical limb darkening profiles for a star with the basic stellar properties of HAT-P-32A
$([\mathrm{Fe} / \mathrm{H}]=-0.04 \pm 0.08, \log g(\mathrm{cgs})=4.33 \pm 0.01$, Hartman et al. 2011 , and $T_{\text {eff }}=6269 \pm 64 \mathrm{~K}$, Zhao et al. 2014) via interpolation from the PHOENIX specific intensity spectra library by Husser et al. (2013). We then weighted each wavelength with its actual contribution to the measured stellar flux during observation, taking into account the instrument response function and the telluric absorption and summed all contributing limb darkening profiles to derive the theoretical white light profile. We then renormalized the model information so that $\mu=0$ actually corresponds to the outer edge of the star, in other words, the region where the mean optical depth corresponds to unity. This is not the case for the raw model data due to the spherical symmetry assumption used in the PHOENIX code. We repeated this process varying the adopted stellar parameters for HAT-P-32A within their reported errors to explore the uncertainties of the intensity profile. Finally, the theoretical limb darkening coefficients and their errors were derived by fitting the intensity profiles with a quadratic limb darkening law. We found that our best-fit limb darkening coefficients were lower than the values predicted by the PHOENIX stellar models $\left(u_{1, \text { theory }}=0.340 \pm 0.056\right.$ and $u_{2, \text { theory }}=0.245 \pm 0.073$ ). This difference might be caused by insufficiencies of the PHOENIX models, errors in the assumed stellar parameters, or undetected systematic noise in the white light curve of Run 1.

\subsection{Consequences for the retrieval of the transmission spectrum of the planet}

Despite the good agreement of our Run 1 white light curve results with the literature data, the lack of extensive out-of-transit data led us to deem the data set unsuitable for a transmission spectroscopy study, where a reliable measurement of very small changes in the transit depth is essential.

Further, we considered the results obtained for the white light curve fit of the Run 2 as unreliable since a clear and dominant systematic noise signal in the data remained uncorrected (see Sect. 3.2). We further investigate this noise signal in Sect. 5 and motivate a correction for the narrowband channel data of Run 2, 


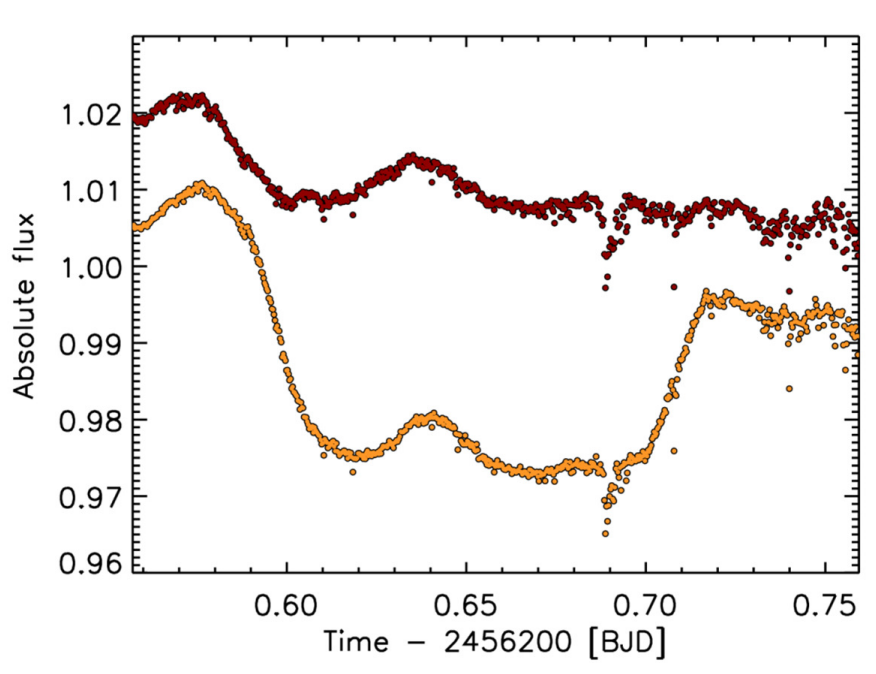

Fig. 9. Raw white light curves of HAT-P-32Ab (yellow) and reference star Ref2 (red) from Run 2.

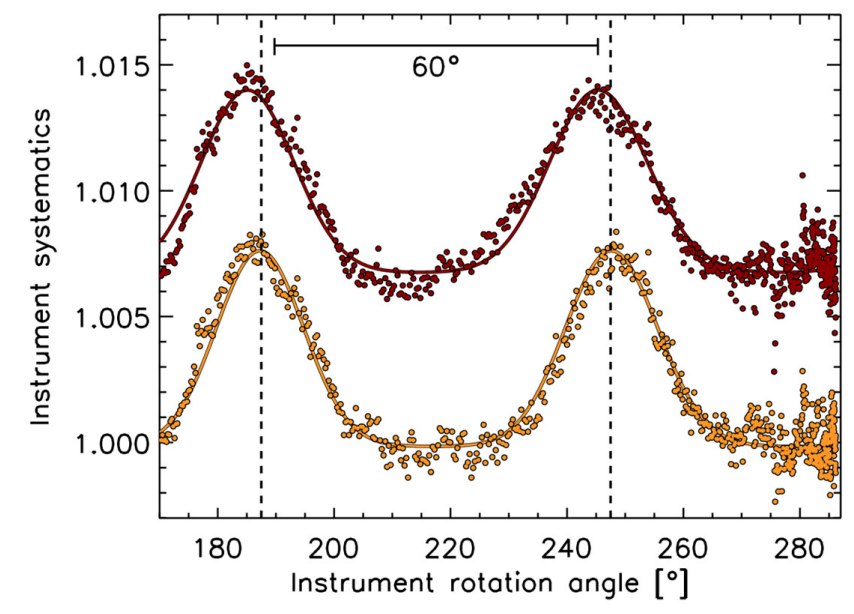

Fig. 10. Raw white light curves of HAT-P-32Ab (yellow circles) and Ref2 (red circles) from Run 2 plotted over the instrument rotation angle for better visualization of the systematic noise signal consisting of two bump-like features. Both curves were corrected by an air mass term and the curve of HAT-P-32A was divided by a theoretical transit. For both raw light curves a rough theoretical approximation of the data consisting of a sequence of two Gaussian functions is plotted over the data (yellow and red lines)

which then can be used to derive a transmission spectrum of HAT-P-32Ab.

\section{GTC/OSIRIS instrument specific systematic noise affecting the data of Run 2}

In Sect. 3 we determined that the systematics affecting the data of Run 2 cannot be sufficiently corrected by solely using a similarly simple decorrelation function of auxiliary parameters as was sufficient for the data of Run 1. The largest nonastrophysical systematic present in the white light curve of Run 2 is the large distortion of the first half of the light curve. It is present in all narrowband channel light curves (see Fig. 15, left panel). When studying the raw white light curves of Run 2 we found that the distortion in the relative light curve coincides with a sinusoidallike feature found in both the raw light curve of HAT-P-32A and the reference star (see Fig. 9). This particular and slightly curious
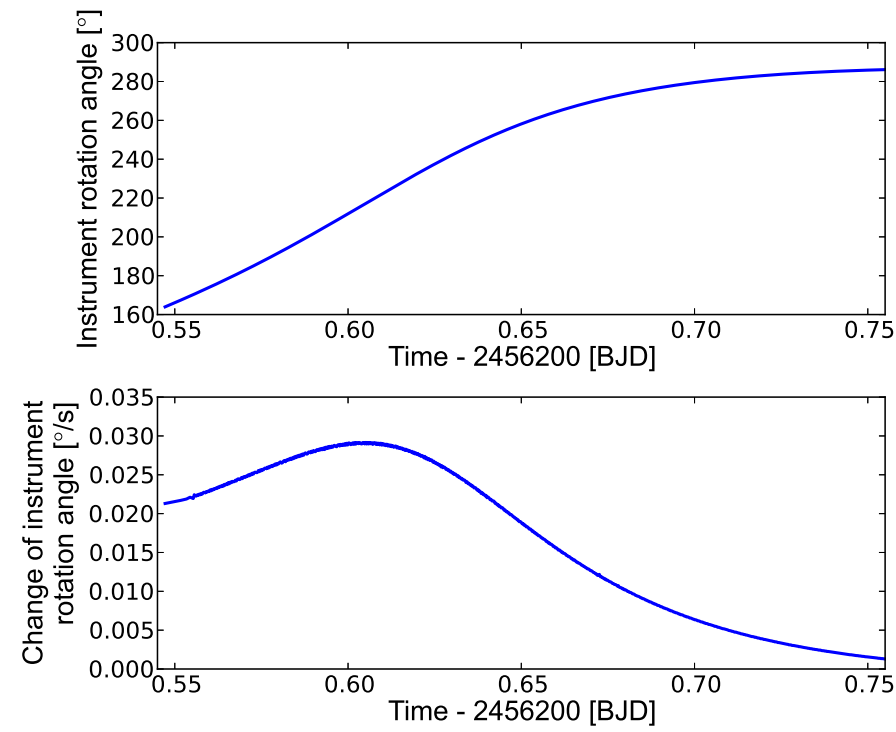

Fig. 11. Top panel: progression of the instrument rotation angle during the second observing night (Run 2). Bottom panel: speed of the changing instrument rotation angle during the second observing night (Run 2). The speed of the instrument rotation slows down in the second half of the observing run.

feature in the first half of the observation does not appear to be caused by telluric atmosphere variations. A closer investigation of the two bumps showed that while they are present in the data of both stars, they are slightly shifted in phase (and possibly have a different amplitude). As a consequence, they do not cancel out when the ratio of both curves is taken to correct any telluric effects. We found that the bumps reappear with a frequency of roughly $60^{\circ}$ of the instrument rotation angle. To better visualize our findings, we divided the raw light curve of HAT-P-32A by a theoretical transit light curve using our best-fit transit parameters from Run 1, cleaned both raw light curves from an air mass trend, and plotted them over the instrument rotation angle (see Fig. 10). We fitted all four bumps with a Gaussian function to derive an estimate for the values of rotation angle at which the peak flux occurs. Since the instrument rotation angle changed during the first half of the observing run and then stayed nearly constant (see Fig. 11) this lead to the bumps only manifesting in the first half of the run. The $60^{\circ}$ symmetry of the bump feature prompted us to suspect these flux amplitude variations to be caused by vignetting in pupil space. A vignetting of the pupil would reduce the overall amount of captured light that reaches the detector. Because of the hexagonal shape of the GTC primary mirror, the pupil is also not invariant under rotation but exhibits a $60^{\circ}$ rotational symmetry. As a consequence, the amount of vignetted pupil area would not only depend on the distance of the projected pupil but also on its effective rotation angle to the source of the vignetting. Vignetting could, for example, occur at the secondary and/or tertiary mirror for off-axis rays. The GTC conceptual design document (Alvarez et al. 1997, Fig. 4.4) indicates that this should only have a significantly impact on targets located at separations larger than $\approx 4^{\prime}$ from the telescope optical axis (which includes Ref2 but not HAT-P-32A in Run 2; see Fig. 1). Potential vignetting caused by other objects located in the optical path in pupil space might, however, also affect targets located closer to the telescope pointing.

We constructed a simple toy model to simulate the expected footprint of pupil vignetting at a hypothetical source. The effect predicted by this toy model exhibited a $60^{\circ}$ periodicity with 


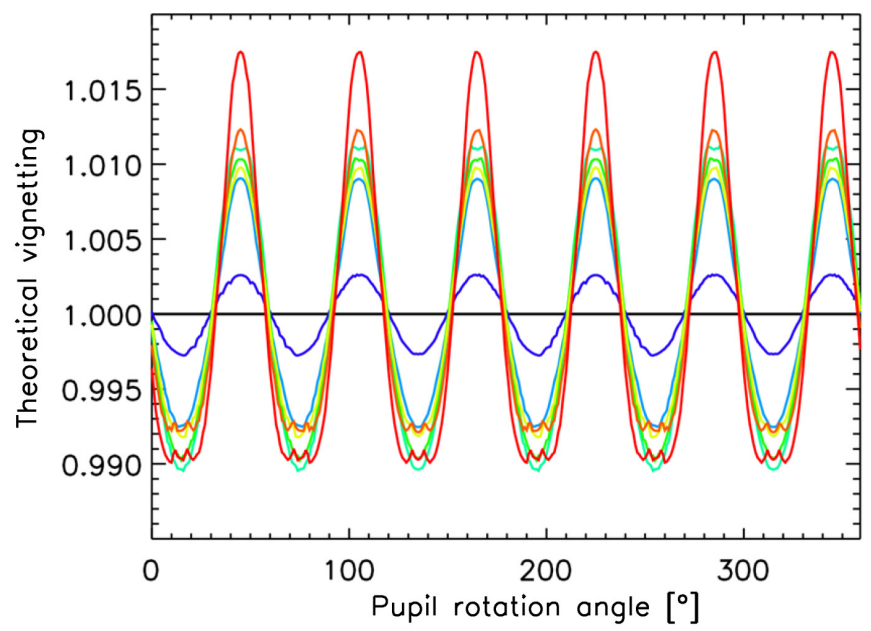

Fig. 12. Theoretical vignetting behavior as a function of effective pupil rotation angle derived from a simple toy model. The different colors represent different distances of the theoretically observed source from the telescope optical center (blue representing the closest distance and red the largest distance).

rotation angle very similar to the signal found in our data of Run 2 (see Fig. 12). The toy model further showed a dependency of the signal on the initial target position relative to the telescope optical axis, which was regulating the phase, amplitude, and precise shape of the effect. The true signal affecting the data could be more complex in case the source of vignetting were located off-center from the telescope optical axis (divergent from the toy model assumption) or if the signal were a superposition of several vignetting signals.

The difference in systematic noise signal amplitude between the two stars of Run 2, which are separated by more than $3^{\prime}(\equiv 1 / 3$ of the OSIRIS field of view) appears to be small, suggesting that for any given observing setup no signals with significantly larger amplitudes (i.e., larger than 1\%) are to be expected. Systematic noise signals of amplitudes slightly smaller than $1 \%$ might be negligible for many other science cases. In our case, however, noise signals of this amplitude are more than two orders larger than the astrophysical signals we are aiming to detect and, therefore, need to be addressed. Since the amount of vignetting is only dependent on the projected pupil position and effective pupil rotation angle, it should be wavelength independent as long as the vignetting occurs before the light has passed any dispersing optical elements. In this case the produced systematic noise signal affects all of the narrowband channel light curves and the white light curve in exactly the same way and should divide out if the ratio between any color light curve and the white light curve is taken.

\section{Analysis of the color light curves of Run 2}

We proceeded to divide all narrowband light curves of Run 2 by the white light curve. This served to cancel out all wavelengthindependent systematic noise signals, that is, signals that affected all curves in an identical manner. Such common-mode corrections are usual practice when dealing with spectrophotometric transit data (Gibson et al. 2013b; Sing et al. 2015). In accordance with expectation, the correction appears to fully dispose of the light curve distortion that we suspect is caused by wavelength-independent pupil vignetting (see Sect. 5). All remaining (i.e., wavelength-dependent) noise signals in the narrowband light curves are found to be linked with auxiliary parameters of the observation in a similar manner as for the white light curve of Run 1 (see Sect. 3). We fitted all differential light curves with a model of the form

$\mathcal{M}_{\mathrm{diff}, n}=\left(\mathcal{T}_{n}+c_{n}\right) /\left(\mathcal{T}_{w}+c_{w}\right) \cdot \mathcal{S}_{n}$,

where $\mathcal{T}_{n}$ is the analytical transit model for the narrowband light curve, $c_{n}$ the dilution factor (i.e., relative flux contribution of the stellar companion HAT-P-32B) in this particular narrowband, $\mathcal{T}_{w}$ the model for the white light transit, $c_{w}$ dilution factor in white light, and $\mathcal{S}_{n}$ a systematic noise model for all remaining wavelength-dependent noise sources. The latter was of the same form as the systematic model used during the white light curve fit, that is, a combination of polynomial functions of the auxiliary parameters detector position, air mass, and FWHM.

Alternatively the original relative light curves can be fitted by a model of the form

$\mathcal{M}_{n}=\left(\mathcal{T}_{n}+c_{n}\right) \cdot \mathcal{S}_{\mathrm{CM}} \cdot \mathcal{S}_{n}$,

where

$\mathcal{S}_{\mathrm{CM}}=\frac{\mathcal{D}_{w}}{\left(\mathcal{T}_{w}+c_{w}\right)}$

is the common mode systematic noise signal derived from the residuals of the white light curve data $\mathcal{D}_{w}$ of Run 2 . This approach is mathematically identical to that given in Eq. (8).

In both cases all wavelength-independent transit model parameters (i.e., $a_{\mathrm{p}} / R_{\star}, i, T_{\mathrm{C}_{2}}, P$, the white light planet-to-star radius ratio, the white light limb darkening coefficients, and the dilution parameters $c_{w}$ and $c_{n}$ ), were kept fixed in this optimization. The values for $c_{n}$ were determined as explained in Appendix A. We chose to fix the white light and wavelength-independent transit parameters to the values we derived from Run 1 in Sect. 3, as they represent a measurement taken with the same instrument and at the same wavelength interval. Not fixing the values but letting them vary in their uncertainty intervals did not change our final results for the relative wavelength-dependent change in radius ratio, and only offset the exact value around which the narrowband radius ratios and limb darkening coefficients varied. The same held true when we fixed the wavelengthindependent and white light transit parameters to the literature values provided by Gibson et al. (2013b), Hartman et al. (2011), or Seeliger et al. (2014). Between these tested configurations adopting our best-fit Run 1 white light parameters did yield the lowest overall $\chi^{2}$. We, therefore, adopted the values resulting from these white light curves values as our final results. To allow an independent use of our derived transmission spectrum, we also provide the relative change in transit depth with respect to the white light curve transit depth, i.e., $\left(R_{\mathrm{p}} / R_{\star}\right)^{2}-\left(R_{\mathrm{p}} / R_{\star}\right)_{\text {white light }}^{2}$, together with our results for the absolute values of the radius ratios in Table 5. These differential transit depths are independent of the white light curve parameters chosen during the analysis and, therefore, free of any possible systematic errors caused by the uncertainties inherent to these parameters.

We again tested all different combinations of systematics models. This time we allowed the highest polynomial order for the air mass and spatial and dispersion position to be 2 and for the FWHM to be 3. The BIC was again calculated using Eq. (3). As we were aiming to determine the model that best explained all 20 channels simultaneously, the joined sum of squared residuals of all 20 light curves (i.e., channel \#1-\#20, excluding the 
Table 4. Model comparison for the systematic noise in the narrowband channel curves of Run 2 listing the respective $\triangle \mathrm{BIC}=\mathrm{BIC}-\mathrm{BIC}_{\min }$ values.

\begin{tabular}{cc}
\hline \hline Model & $\Delta$ BIC \\
\hline xpos0 ypos1 airm1 fwhm2 & 114.82 \\
xpos0 ypos1 airm1 fwhm3 & 211.20 \\
xpos0 ypos2 airm1 fwhm2 & 282.08 \\
xpos1 ypos1 airm1 fwhm2 & $\mathbf{0 . 0 0}$ \\
xpos1 ypos1 airm1 fwhm3 & 77.08 \\
xpos1 ypos2 airm1 fwhm2 & 170.87 \\
xpos1 ypos2 airm1 fwhm3 & 240.33 \\
xpos2 ypos1 airm1 fwhm2 & 179.62 \\
xpos2 ypos1 airm1 fwhm3 & 255.41 \\
xpos2 ypos2 airm1 fwhm2 & 350.57 \\
xpos2 ypos2 airm1 fwhm3 & 418.44 \\
\hline
\end{tabular}

subchannel \#13b) was used in the BIC calculation. The overall lowest BIC was reached with the model

$$
\begin{aligned}
\mathcal{S}_{n}= & n_{0}+x_{1} \cdot \text { xpos }+y_{1} \cdot \text { ypos }+a_{1} \cdot \text { airm } \\
& +f_{1} \cdot f w h m+f_{2} \cdot f w h m^{2}
\end{aligned}
$$

where $n_{0}, x_{1}, y_{1}, a_{1}$, and $f_{1,2}$ are wavelength-dependent parameters and, therefore, different for every channel. An overview of the BIC comparison can be found in Table 4 in which all models with a $\triangle \mathrm{BIC}<1000$ are listed. When investigating the individual channels separately we found that most of the color light curves were satisfied with a less complex model without a spatial position (xpos) dependent term. The $\chi^{2}$ of the three bluest channels, however, was significantly minimized by introducing this additional parameter, leading to an overall favored BIC without affecting the final results for the other channels.

The results from the different models listed in Table 4 are plotted in Fig. 13 together with the values we adopted as our final results. It can be seen that at the blue end of the spectrum a few models give notably different results. The models yielding such deviant results are, however, not those favored when determining the best model for each channel individually. The resulting radius ratios for the individually determined best models (red squares) are in good agreement with the adopted results derived from the homogeneous analysis.

Errors. We repeated the exploration of the posterior parameter distributions with MCMC as described for the white light curve analysis in Sect. 3.1, now letting the values for the wavelengthdependent dilution factors $c_{n}$ vary within their uncertainty intervals using Gaussian priors. This time we used 300 walkers and let them run for 10000 accepted steps. We discarded all points of the burn-in period, which was over after 1000 steps. The typical thinning factors for the chains were $\approx 82$, leaving us with a final distribution of an average of 33300 points for each wavelength channel light curve fit. An example of the resulting posterior parameter distributions is shown for channel \#9 in Fig. 14. We then calculated the red noise factor for every narrowband channel light curve as described in Sect. 3.2 and inflated the MCMC derived error bars accordingly. The resulting values for $\beta$ lie between 1.022 and 1.950 and the resulting uncertainties of the wavelength-dependent radius ratio after multiplication with $\beta$ lie between 337 and 972 ppm. The light curves before and after the full systematic noise correction are shown in Fig. 15. The

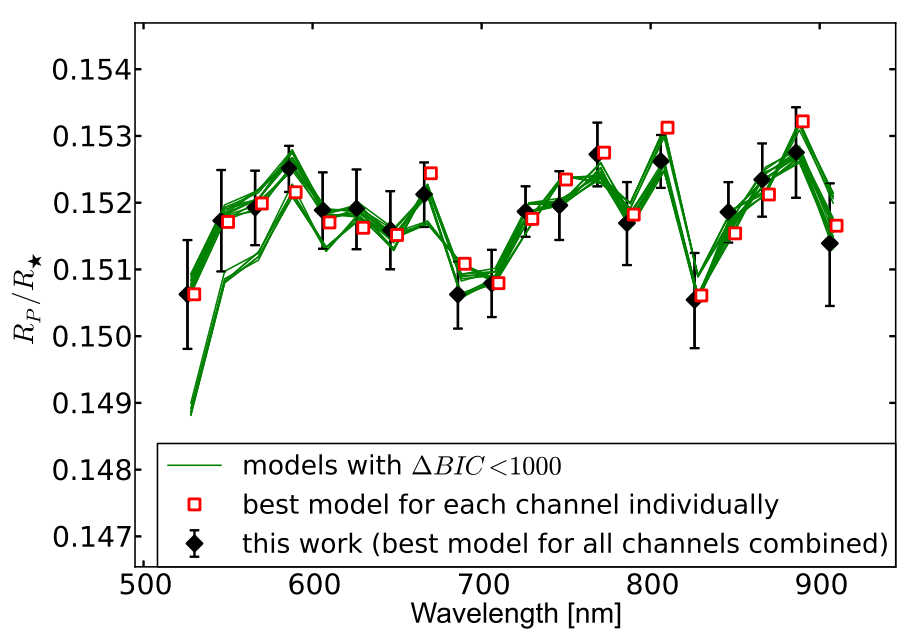

Fig. 13. Results for the wavelength-dependent, radius ratios obtained for the different models listed in Table 4 (green lines). The final adopted results, calculated with a homogeneous analysis, are overlayed as black diamonds (error bars are calculated as described in Sect. 6). The results obtained when using the individually best-suited model for each wavelength channel are plotted as red squares.

resulting radius ratios and limb darkening coefficients as well as $\beta$ factors are given in Table 5 .

\section{Results and discussion of the transmission spectrum}

The result for the wavelength-dependent, planet-to-star radius ratio of HAT-P-32Ab is shown in Fig. 16. We find that it shows little variation in the probed region between 518 and $918 \mathrm{~nm}$. The variations are significantly smaller than two planetary atmospheric scale heights $\left(H_{\mathrm{p}} \approx 1400 \mathrm{~km}\right)$. This estimate for $H_{\mathrm{P}}$ is derived with Eq. (12),

$H_{\mathrm{p}}=\frac{k_{b} T_{\mathrm{p}}}{m g_{\mathrm{p}}}$

using the planet equilibrium temperature $T_{\mathrm{p}}=2042 \mathrm{~K}$ given by Zhao et al. (2014), the planetary gravitational acceleration of $g_{\mathrm{p}}=6.6069 \mathrm{~m} / \mathrm{s}^{2}$ from Hartman et al. (2011), and the approximation of the mean molecular weight of the atmosphere $m$ as that of a solar abundance hydrogen helium mixture. In Eq. (12), $k_{\mathrm{B}}$ stands for the Boltzmann constant. The best-fit results for the limb darkening coefficients are shown in Fig. 17. They vary smoothly with wavelength except for the coefficients for channel \#13, the channel encompassing the telluric oxygen bands at $\approx 761 \mathrm{~nm}$. We compare the results for the limb darkening coefficients with theoretical predictions. Our approach to the narrowband channel analysis entailed division by the white light curve. Therefore, effectively only relative changes of the transit depth and stellar limb darkening to the white light transit depth and white light limb darkening were measured. Consequently, the absolute values of our resulting color-dependent limb darkening values are affected by the assumed white light limb darkening values. Since we adopted the best-fit white light curve parameters from Run 1, where the limb darkening coefficients are divergent from theoretical expectations, the resulting color-dependent coefficients are by default divergent as well. To compare these coefficients to the theoretical expectations independently, we calculated modified theoretical values that correspond to the theoretical predictions for the color-dependent coefficients under assumption of the white light coefficients fixed 


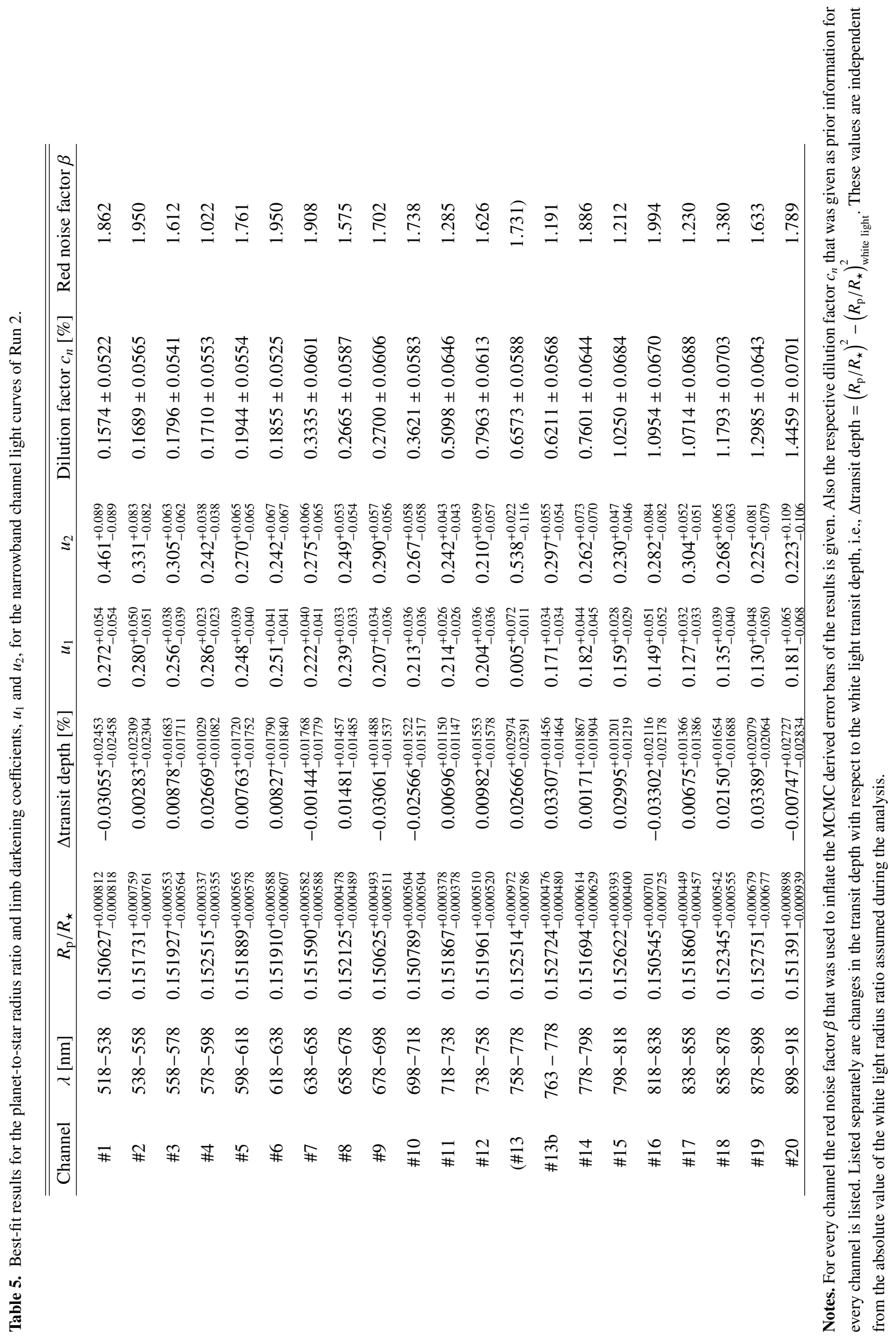




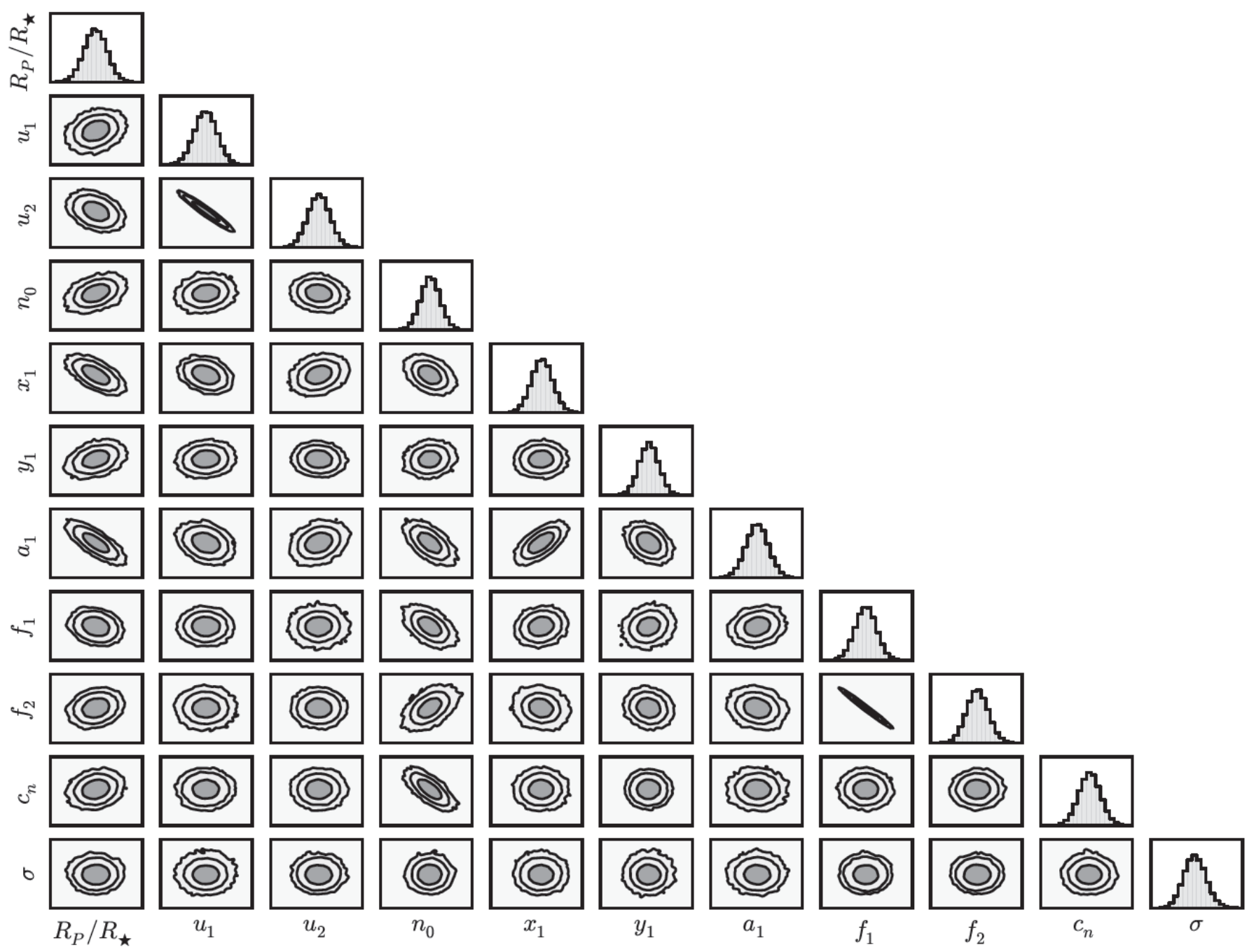

Fig. 14. Example correlation plots for the posterior parameter distributions for the fit of narrowband channel \#9 (678 nm-698 nm). The lines indicate the areas encompassing $1 \sigma, 2 \sigma$, and $3 \sigma$ of the whole distribution, respectively.

to the best-fit results of Run 1 . These modified theoretical values were derived from PHOENIX specific intensity spectra as described in Sect. 4.2, where instead of the whole white light wavelength region, now only the corresponding narrowband channels were summed to derive the limb darkening profile. The resulting modified theoretical prediction is shown together with the actual measured limb darkening coefficients in Fig. 17. It can be seen that the strong change in limb darkening in respect to the neighboring wavelength regions that we see for channel \#13 is not expected from theory. Since the results for subchannel \#13b do not show the same divergence from theory, we conclude that the results of channel \#13 are affected by uncorrected noise due to the strong telluric absorption bands and are unreliable. Channel \#13 does not carry any significant information that is not also represented by channel \#13b. Therefore, we decide to exclude the results for the planet-to-star radius ratio of channel \#13 in the following comparison to literature data and planet atmosphere model predictions.

\subsection{Comparison to theoretical models and literature data}

We compared the results to theoretical atmosphere models with various degrees of alkali metal abundance depletion (see
Fig. 18). The atmospheric models were calculated with a lineby-line radiative code described in Iro et al. (2005) with updated opacities described in Agúndez et al. (2012) and Montalto et al. (2015). We assumed a clear atmosphere (no clouds) in thermochemical equilibrium with a solar abundance of elements for the nominal model. Furthermore, other models with various changes in titanium oxide (TiO) and alkali abundances were explored. The stellar heating was provided using a stellar synthetic spectrum $^{1}$ of a G-type star with an effective temperature of $6000 \mathrm{~K}$ from Castelli \& Kurucz (2003). We found no agreement between the measured transmission spectrum and the expected absorption features of the sodium and potassium resonance doublets ( $\mathrm{Na}$ I at 589.0 and $589.6 \mathrm{~nm}, \mathrm{KI}$ at 766.5 and $769.9 \mathrm{~nm}$, where the doublets are unresolved at our resolution) predicted by the nominal model (see top panel of Fig. 18). The radius ratio in channel \#4 encompassing the sodium doublet is lower by $\approx 12.9 \sigma$ than the predicted value and the radius ratio in channel \#13b, encompassing the potassium doublet, is lower by $\approx 7.4 \sigma$. Further, there is no indication of a detection of the predicted broad wings of the sodium feature in the channels neighboring channel \#4. Neither an enhancement of the titanium oxide abundance in the probed upper layers of the atmosphere (see top panel of

1 Available at ftp://ftp.stsci.edu/cdbs/grid/ck04models 


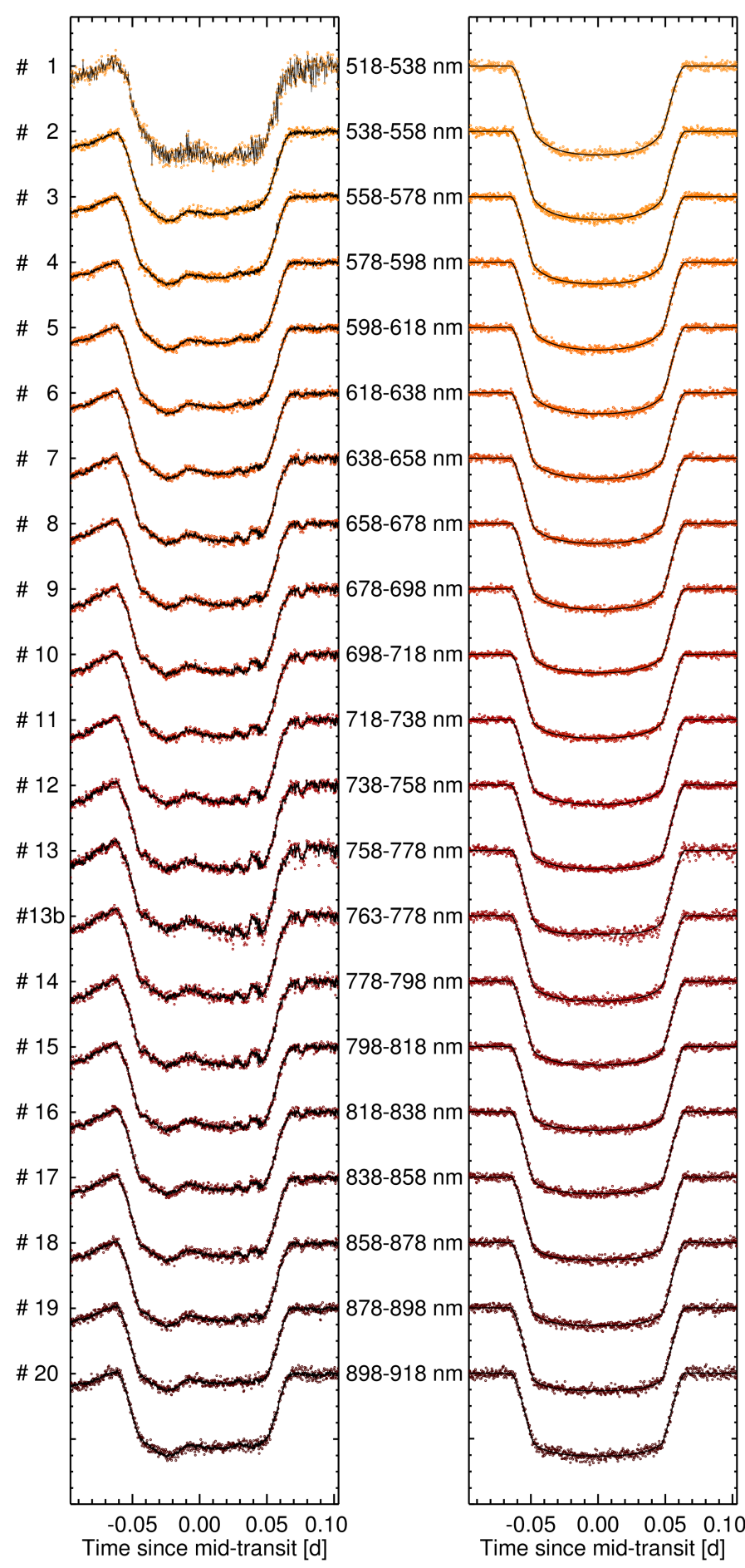

Fig. 15. All narrowband channel light curves before and after correction from the systematic noise signals plotted together with the best-fit model (black line).

Fig. 18) nor a depletion of either only sodium or only potassium (see middle panel of Fig. 18) improves the fit between the model and data. Reducing the abundance of both these alkali metals by a factor of 1000 yields a model that agrees fairly well with the data with a reduced $\chi^{2}$ of 1.58 (20 degrees of freedom). This is a similar but slightly worse match than the match to a straight line representative of a gray atmosphere (see bottom panel of Fig. 18), which results in a reduced $\chi^{2}$ of 1.50 (20 degrees of freedom). A gray atmosphere signal could be caused

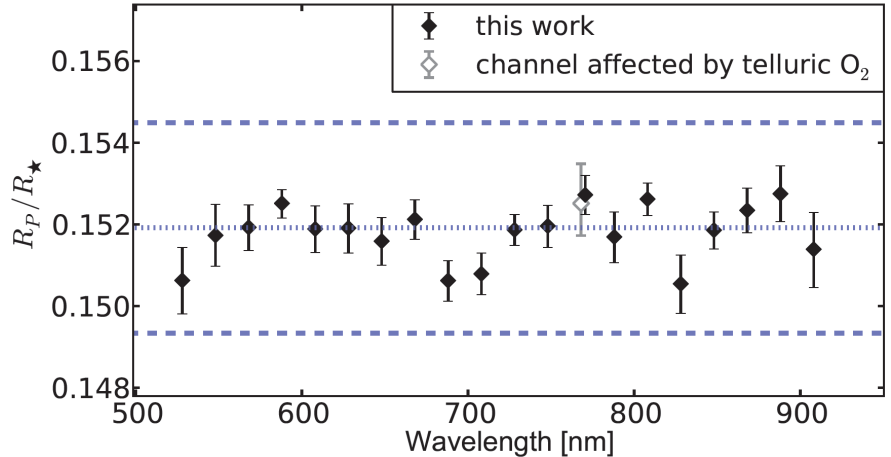

Fig. 16. Results for the wavelength-dependent, planet-to-star radius ratio of HAT-P-32Ab from Run 2. The blue dotted line indicates the mean radius ratio and the blue dashed lines indicate \pm two atmospheric scale heights.
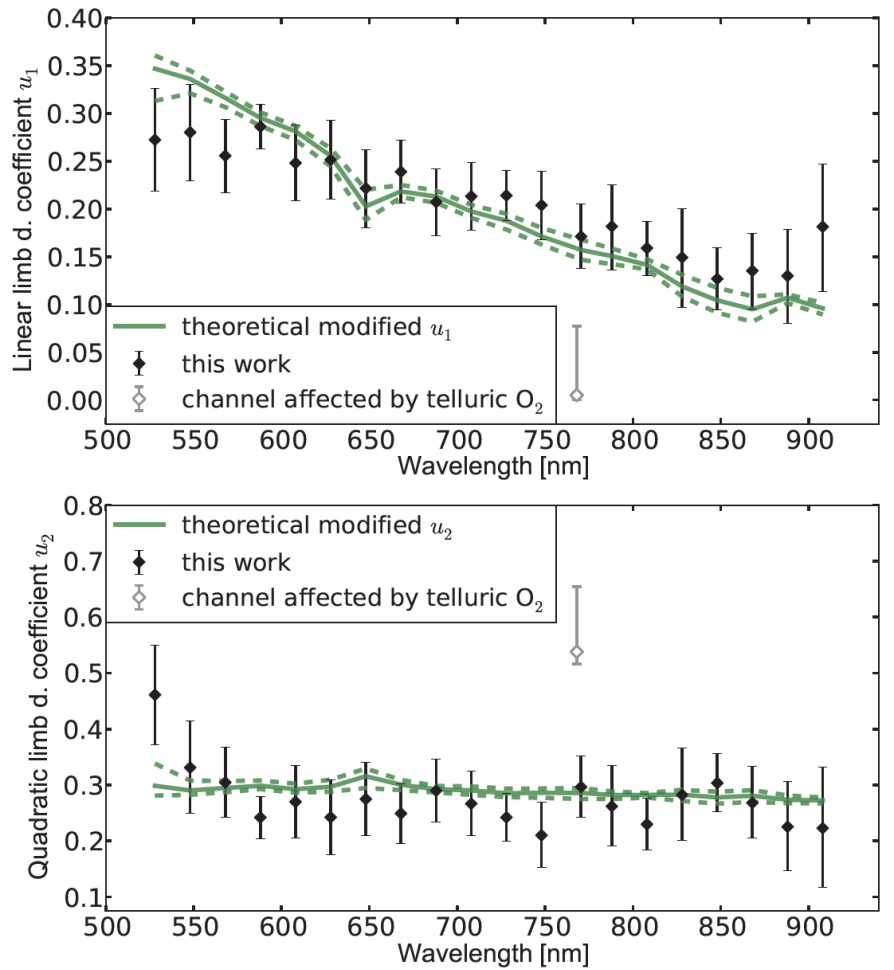

Fig. 17. Progression of the results for the linear $\left(u_{1}\right.$, top panel) and quadratic $\left(u_{2}\right.$, bottom panel $)$ limb darkening coefficients with wavelength from Run 2. Both are compared with a theoretical prediction derived from PHOENIX models (green line) and its error (green dashed line) taking the uncertainties in the stellar parameters of the host star HAT-P-32A into account.

by a high altitude cloud layer masking the fingerprint of the atmosphere below. Alternatively, a significantly lower atmospheric scale height of the terminator region than the one predicted by the measured equilibrium temperature of the planet could be responsible. The amplitude of all expected atmospheric features would shrink accordingly and in an extreme case they would be hidden in the uncertainties of the measurement. To be in very good agreement with the data, the scale height would have to be lower than the current prediction by a factor of 6.8. Such a decrease in scale height could, for example, be caused by either a lower than predicted terminator temperature $(\approx 300 \mathrm{~K})$ or an increase of the assumed mean molecular weight by that factor (i.e., $m \approx 16 \mathrm{~kg} / \mathrm{kmole}$; for comparison the mean molecular weight of 

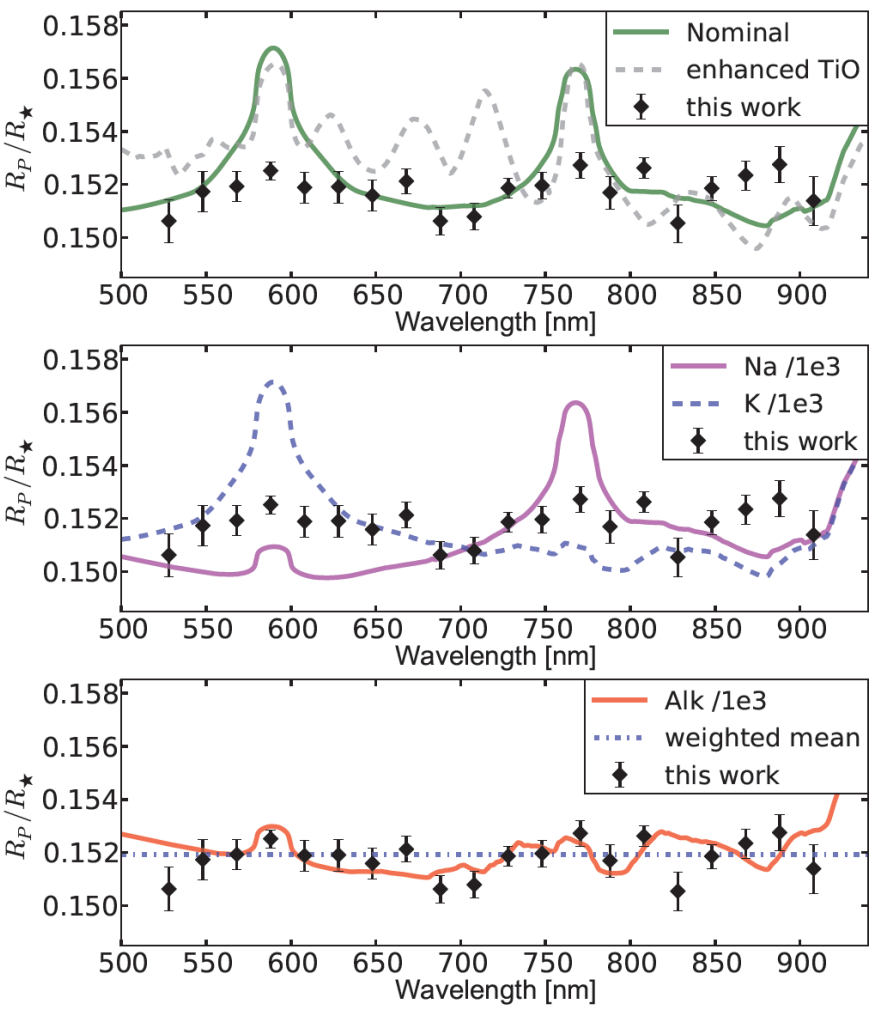

Fig. 18. Results for the wavelength-dependent planet radius of HAT-P$32 \mathrm{Ab}$ from Run 2 (circles) compared with several different theoretical atmosphere models. Top panel: unmodified solar composition model at thermochemical equilibrium (green) and the same model with the titanium oxide ( $\mathrm{TiO})$ abundance enhanced in the upper layers of the atmosphere (gray dashed). Middle panel: models with selective alkali metal abundance depletion. In one model, only the sodium abundance was reduced by a factor of 1000 (magenta) and in the other only the potassium abundance was reduced by a factor of 1000 (blue dashed). Bottom panel: model where both the sodium and potassium abundance were reduced by a factor of 1000 (red) and the median average of the radius ratio values (blue dash-dotted).

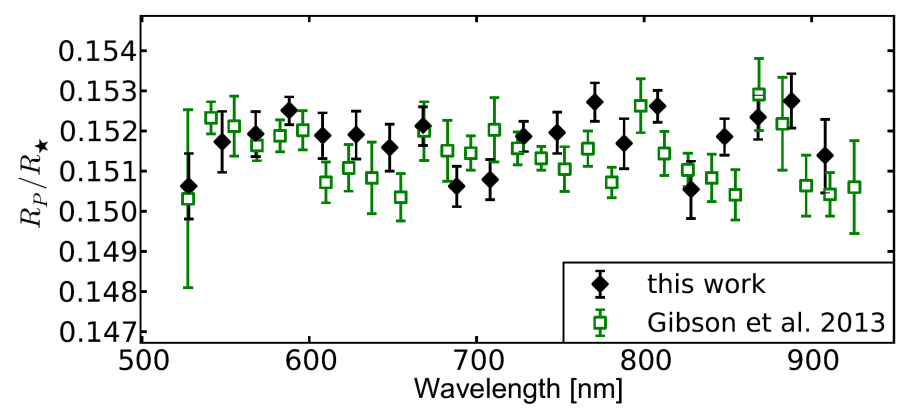

Fig. 19. Results for the wavelength-dependent planet radius of HAT-P$32 \mathrm{Ab}$ from Run 2 compared with the results from Gibson et al. (2013b).

water vapor is $\left.m_{\mathrm{H}_{2} \mathrm{O}}=18.02 \mathrm{~kg} / \mathrm{kmole}\right)$. A more plausible explanation, however, would be that both of these two regulating factors are divergent from the values assumed in the current scale height calculation.

Our results for the planet-to-star radius ratio are in good agreement with the study of Gibson et al. (2013b) who observed two transit events of HAT-P-32Ab with GMOS at Gemini North. They analyzed both data sets separately and then combined the final results for the planet-to-star radius ratio of both data sets. In Fig. 19 we compare our results with their combined results.

\subsection{Potential of GTC/OSIRIS as a tool for transmission spectroscopy}

We found the light curves to be heavily affected by systematics, which can be modeled, however. Only the channel containing the telluric oxygen absorption band at $\approx 761 \mathrm{~nm}$ was too heavily affected by noise to be sufficiently corrected. The data showed correlation with the position of the stars on the chip (and in the slit) with air mass, which is expected because of the difference in color between the planet host star and reference star, and with seeing. The latter correlation suggests slit losses that affect the planet host star and reference star differently. This problem can be overcome by choosing a larger slit width. We adopted this for the following runs (e.g., Parviainen et al. 2016). While the rotation dependent distortion cannot be overcome, it affects the data less when the changes in the rotation angle are small. It also has a smaller impact when the two stars are close to each other on the chip since vignetting should then be similar for both stars. In such cases, that is, when no obvious distortions can be seen in the data, disregarding their possible hidden existence could lead to systematically erroneous transit parameters, but should have negligible impact on the derived transmission spectrum as long as only relative variations in the radius ratio are considered.

\section{Conclusion}

We were able to derive a high precision transmission spectrum for the inflated hot Jupiter HAT-P-32Ab showing no prominent absorption features and, thus, supporting the results of the earlier work by Gibson et al. (2013b). The study allowed us to detect and understand the low-level instrument systematics affecting GTC/OSIRIS and will help to improve future measurements. The independent confirmation of ground-based results from a different ground-based facility affected by different systematic noise signals is a step toward re-establishing faith in the reliability of (ground-based) transmission spectroscopy measurements. We have confidence in the potential of ground-based facilities and GTC/OSIRIS, in particular, as an excellent tool for larger surveys.

Acknowledgements. This work is based on observations made with the Gran Telescopio Canarias (GTC) installed in the Spanish Observatorio del Roque de los Muchachos of the Instituto de Astrofisica de Canarias in the island of La Palma. It is partly financed by the Spanish Ministry of Economics and Competitiveness through projects ESP2013-48391-C4-2-R and ESP2014-57495-C21-R. L.N. acknowledges support from the DFG Graduiertenkolleg 1351 Extrasolar Planets and their Host Stars. F.M. acknowledges the support of the French Agence Nationale de la Recherche (ANR), under the program ANR-12-BS050012 Exo-atmos. Many of the plots shown in this paper were made using Matplotlib (Hunter 2007).

Note added in proof. We would like to note that during a late stage of this paper's referee process another study of HAT-P-32b by Mallonn \& Strassmeier (2016) was uploaded to ArXiv. This study further confirms the measurement of a flat transmission spectrum for this planet's atmosphere.

\section{References}

Acosta-Pulido, J., Ballesteros, E., Barreto, M., et al. 2002, The Newsletter of the Isaac Newton Group of Telescopes, 6, 22

Adams, E. R., Dupree, A. K., Kulesa, C., \& McCarthy, D. 2013, AJ, 146, 9

Agúndez, M., Venot, O., Iro, N., et al. 2012, A\&A, 548, A73

Alvarez, P., et al. 1997, Gran Telescopio Canarias Conceptual design, GTC Project Document GEN/STMA/0012-L, Grantecan, La Laguna

Baranec, C., Riddle, R., Law, N. M., et al. 2014, ApJ, 790, L8 
Bean, J. L., Miller-Ricci Kempton, E., \& Homeier, D. 2010, Nature, 468, 669 Berta, Z. K., Charbonneau, D., Désert, J.-M., et al. 2012, ApJ, 747, 35

Castelli, F., \& Kurucz, R. L. 2003, in Modelling of Stellar Atmospheres, eds N. Piskunov, W. W. Weiss, \& D. F. Gray, IAU Symp., 210, 20

Charbonneau, D., Brown, T. M., Noyes, R. W., \& Gilliland, R. L. 2002, ApJ, 568,377

Davies, R. L., Allington-Smith, J. R., Bettess, P., et al. 1997, in Optica Telescopes of Today and Tomorrow, ed. A. L. Ardeberg, Proc. SPIE, 2871 1099

Désert, J.-M., Lecavelier des Etangs, A., Hébrard, G., et al. 2009, ApJ, 699, 478

Eastman, J., Gaudi, B. S., \& Agol, E. 2013, PASP, 125, 83

Ehrenreich, D., Hébrard, G., Lecavelier des Etangs, A., et al. 2007, ApJ, 668, L179

Foreman-Mackey, D., Hogg, D. W., Lang, D., \& Goodman, J. 2013, PASP, 125, 306

Gibson, N. P., Pont, F., \& Aigrain, S. 2011, MNRAS, 411, 2199

Gibson, N. P., Aigrain, S., Barstow, J. K., et al. 2013a, MNRAS, 428, 3680

Gibson, N. P., Aigrain, S., Barstow, J. K., et al. 2013b, MNRAS, 436, 2974

Hartman, J. D., Bakos, G. Á., Torres, G., et al. 2011, ApJ, 742, 59

Horne, K. 1986, PASP, 98, 609

Hunter, J. D. 2007, Comput. Sci. Eng., 9, 90

Husser, T.-O., Wende-von Berg, S., Dreizler, S., et al. 2013, A\&A, 553, A6

Iro, N., Bézard, B., \& Guillot, T. 2005, A\&A, 436, 719

Jordán, A., Espinoza, N., Rabus, M., et al. 2013, ApJ, 778, 184
Kashyap, V. L., \& Drake, J. J. 2000, BAAS, 32, 1227

Knutson, H. A., Fulton, B. J., Montet, B. T., et al. 2014, ApJ, 785, 126

Mallonn, M., \& Strassmeier, K. G. 2016, A\&A, 590, A100

Mallonn, M., von Essen, C., Weingrill, J., et al. 2015, A\&A, 580, A60

Mandel, K., \& Agol, E. 2002, ApJ, 580, L171

Markwardt, C. B. 2009, in Astronomical Data Analysis Software and Systems XVIII, eds. D. A. Bohlender, D. Durand, \& P. Dowler, ASP Conf. Ser., 411, 251

Mayor, M., \& Queloz, D. 1995, Nature, 378, 355

Montalto, M., Iro, N., Santos, N. C., et al. 2015, ApJ, 811, 55

Murgas, F., Pallé, E., Zapatero Osorio, M. R., et al. 2014, A\&A, 563, A41

Ngo, H., Knutson, H. A., Hinkley, S., et al. 2015, ApJ, 800, 138

Parviainen, H., Pallé, E., Nortmann, L., et al. 2016, A\&A, 585, A114

Pont, F., Knutson, H., Gilliland, R. L., Moutou, C., \& Charbonneau, D. 2008, MNRAS, 385, 109

Sánchez, B., Aguiar-González, M., Barreto, R., et al. 2012, in SPIE Conf. Ser., 8446,4

Seeliger, M., Dimitrov, D., Kjurkchieva, D., et al. 2014, MNRAS, 441, 304

Sing, D. K., Huitson, C. M., Lopez-Morales, M., et al. 2012, MNRAS, 426, 1663

Sing, D. K., Wakeford, H. R., Showman, A. P., et al. 2015, MNRAS, 446, 2428

Tinetti, G., Vidal-Madjar, A., Liang, M.-C., et al. 2007, Nature, 448, 169

Winn, J. N., Holman, M. J., Torres, G., et al. 2008, ApJ, 683, 1076

Wizinowich, P., Acton, D. S., Shelton, C., et al. 2000, PASP, 112, 315

Zhao, M., O'Rourke, J. G., Wright, J. T., et al. 2014, ApJ, 796, 115 


\section{Appendix A: Companion HAT-P-32B}

In 2013 Adams et al. discovered an optical companion to HAT$\mathrm{P}-32 \mathrm{~A}$ using adaptive optics (AO) with Arizona Infrared imager and Echelle Spectrograph (Aries, Principle Investigator: Don McCarthy), which is fed by the Multiple Mirror Telescope's (MMT) AO beam. The optical companion was later confirmed to be bound to the HAT-P-32 system by Ngo et al. (2015) from proper motion measurements and AO imaging. HAT-P-32B was further characterized as an M-dwarf by Zhao et al. (2014) and Ngo et al. (2015) who used near-infrared broadband AO imaging to constrain its stellar parameters.

Because of the close proximity of the two stars, HAT-P-32A and $\mathrm{B}$, the flux of the latter contributed to our measurements of the former. We needed to determine the wavelength-dependent flux ratio between the two stars as precisely as possible to correctly include this effect in our models. In the following, we describe how we extracted this information from our GTC/OSIRIS spectra. Subsequently, we use this data and additionally obtained near-infrared observations to derive improved stellar parameters for HAT-P-32B.

\section{A.1. Optical spectrum - GTC/OSIRIS}

Since HAT-P-32B was undiscovered prior to our observations the observing setup was not optimized to maximize the projected distance of the two spectra of HAT-P-32A and B on the chip. We still were able to detect HAT-P-32B as a separated object in Run 2 as a deformation of the spatial profile of HAT-P-32A.

Using the out-of-transit data available in Run 2 we determined the flux peak of the HAT-P-32A spectrum in every frame at every wavelength and then added frames in 10-frame time bins resulting in 25 images with increased signal to noise. We then fitted a double-peak model based on an empirical profile function to the spatial profiles of HAT-P-32A and B for every wavelength cut though the spectrum for all 25 images. We used an empirical profile based on a Moffat function and assumed symmetry of the stellar profile. It is described in detail in Sect. A.1.1. A sample fit to the spatial double profile can be found in Fig. A.1.

\section{A.1.1. Empirical profile}

We acquired a first approximation of the peak position and amplitude of the two stellar profiles by fitting each of them with a Moffat function enforcing identical values for the half widths and for the Moffat indices of both profiles. The result showed that the wings of the actual stellar profiles were underestimated by the Moffat approximation. Consequently, we moved on to an empirical approach. For this we assumed that the actual profile is symmetrical with respect to its central axis and that the point spread functions (PSFs) of both stars are the same. We constructed an empirical profile, $\mathcal{P}_{\mathrm{e}}$, using the side of the stellar profile of HAT-P-32A, which is not distorted by the additional flux of HAT-P-32B, and mirroring it at the central axis of the HAT-P-32A profile. We then used a scaled down, shifted in pixel position version of $\mathcal{P}_{\mathrm{e}}$ to model the profile of HAT-P-32B. The final model for the superposition of both stellar profiles $\mathcal{W}$ was of the form

$\mathcal{W}(x)=\mathcal{P}_{\mathrm{e}}(x)+s \cdot \mathcal{P}_{\mathrm{e}}\left(x-x_{\mathrm{A}-\mathrm{B}}\right)$.

In this model the scaling factor $(s)$, the center of the stellar profile of HAT-P-32A $(x)$, and the distance of the centers of both stars $\left(x_{\mathrm{A}-\mathrm{B}}\right)$ were free parameters. From the optimization of this model we obtained the flux for HAT-P-32A $\left(f_{\mathrm{A}}\right)$ and HAT-P-32B

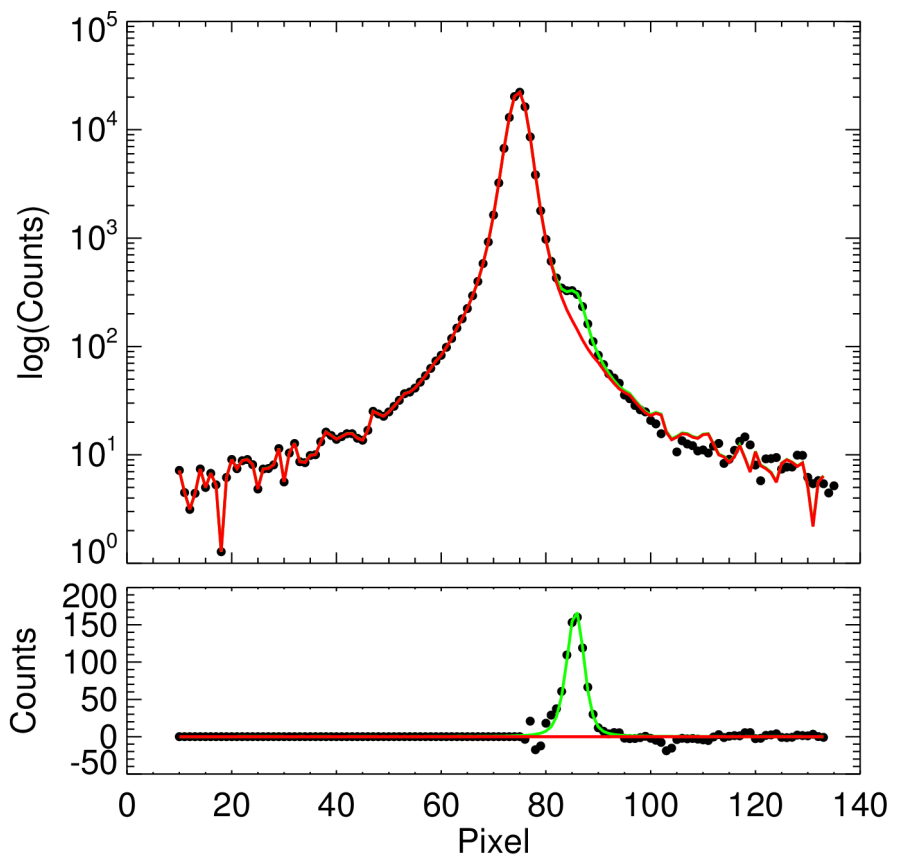

Fig. A.1. Top panel: example for the combined stellar profiles of HAT$\mathrm{P}-32 \mathrm{~A}$ and HAT-P-32B plotted in semi logarithmic scale. In red the empirical model profile for HAT-P-32A is shown. The green line indicates where the combined profile diverges from HAT-P-32A's profile. Bottom panel: same data as in top panel after subtraction of the empirical model profile of HAT-P-32A. The green line now represents the model profile of HAT-P-32B.

$\left(f_{\mathrm{B}}\right)$ for every wavelength element in each of the 25 images by summing over the respective theoretical profiles.

\section{A.1.2. Dilution of the optical transit depth measurements of HAT-P-32Ab}

Since HAT-P-32B was within the aperture used in Sect. 2.1 to determine the flux of HAT-P-32A, its additional flux had a diluting effect on the transit depth. This effect is color dependent as HAT-P-32B contributes different amounts of flux in the individual wavelength channels defined in Sect. 2.2. We determined the contribution in every wavelength channel by first summing the measured flux of both stars separately within the channel limits in each of the 25 frames. For each channel we then divided the resulting total flux of both stars and adopted the mean of the 25 flux ratios as the final result of the dilution factor $c_{n}=f_{\mathrm{A}} / f_{\mathrm{B}}$ and their standard deviation as its uncertainty. The resulting values for each channel are listed in Table 5 in Sect. 7.

\section{A.1.3. Resulting relative optical spectrum}

While for the correction of the multicolor channel light curves in this paper only low resolution information for HAT-P-32b was needed, higher resolution information was available. We used this data to determine the stellar properties of HAT-P-32B in Sect. A.4. In order to remove the OSIRIS instrument sensitivity function from the data and clean it of any telluric absorption, we used the relative spectrum of HAT-P-32B and HAT-P-32A for this analysis. Consequently, all wavelength-dependent telluric and instrumental effects are divided out. Since the slit alignment was optimized to center HAT-P-32A and the reference star (Ref2, see Table 1) within it, HAT-P-32B was not perfectly 

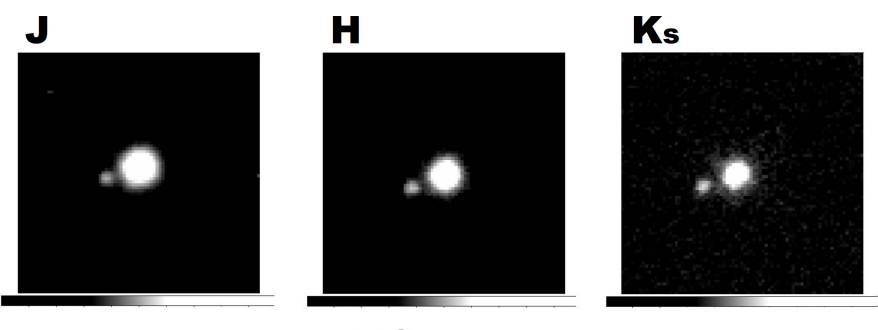

$\mapsto 2$ arcsec

Fig. A.2. $J, H, K_{\mathrm{S}}$ band photometry of HAT-P-32B and HAT-P-32A obtained with LIRIS at the WHT.

Table A.1. Flux ratios between HAT-P-32B and HAT-P-32A obtained from infrared imaging in this work, Ngo15 (Ngo et al. 2015), Zhao14 (Zhao et al. 2014), and Adams13 (Adams et al. 2013).

\begin{tabular}{ccccc}
\hline \hline $\begin{array}{c}f_{\mathrm{B}} / f_{\mathrm{A}}[\%] \\
\text { in filter }\end{array}$ & This work & Zhao14 & $\mathrm{Ngo15}^{a}$ & Adams13 $^{a}$ \\
\hline$J$ & $2.65 \pm 0.45$ & $\ldots$ & $2.19_{-0.56}^{+0.57}$ & $\ldots$ \\
$H$ & $3.73 \pm 0.65$ & $4.4 \pm 0.5$ & $3.41_{-0.22}^{+0.23}$ & $\ldots$ \\
$K^{\prime}$ & $\ldots$ & $\ldots$ & $3.21_{-0.12}^{+0.13}$ & $\ldots$ \\
$K_{\mathrm{S}}$ & $4.64 \pm 0.21$ & $4.7 \pm 0.2$ & $3.98_{-0.26}^{+0.28}$ & 0.044 \\
\hline
\end{tabular}

Notes. ${ }^{(a)}$ Value calculated from the relative magnitudes $\Delta$ mag given in the paper.

centered in the slit. As a consequence, the wavelength solution for its spectrum is slightly shifted compared to the wavelength solution of HAT-P-32A. We first applied this shift in wavelength and then calculated the flux ratio for every wavelength element in each of the 25 frames. We again adopted the mean of the 25 results as the final value and their standard deviation as the uncertainty. The resulting relative spectrum, covering the wavelength range $518-918 \mathrm{~nm}$, is shown in Fig. A.3.

\section{A.2. Infrared photometry - WHT/LIRIS}

In addition to the optical spectrum we obtained $J, H$, and $K_{\mathrm{S}}$ band measurements with the William Herschel Telescope (WHT) in the night of 6 October 2012 using the Longslit Intermediate Resolution Infrared Spectrograph (LIRIS; Acosta-Pulido et al. 2002) in imaging mode. We took 18 images and obtained 15 flats and darks 15 for each filter. A sample image of these observations for each filter is shown in Fig. A.2. We used PSF fitting to obtain the absolute flux for HAT-P-32B and HAT-P-32A. We then calculated the flux ratio $f_{\mathrm{B}} / f_{\mathrm{A}}$ for every image and adopted the mean as the final value and the standard deviation as the uncertainty for each filter. The results are listed in the first column of Table A.1.

\section{A.3. Comparison to literature}

We compared our results of the flux ratio $c_{\text {filter }}=f_{\mathrm{A}} / f_{\mathrm{B}}$ of HAT-P-32B and HAT-P-32A with the broadband measurements from the literature obtained in the optical $\left(g^{\prime}, r^{\prime}, i^{\prime}, z^{\prime}\right)$ by Zhao et al. (2014) with the Robo-AO instrument (Baranec et al. 2014) on the 60 inch telescope at the Palomar Observatory and in the near-infrared $\left(J, H, K^{\prime}\right.$ and $\left.K s\right)$ by Zhao et al. (2014) and Ngo et al. (2015) with NIRC2 a near-infrared imager
(Principle Investigator: Keith Matthews) using the AO system of Keck-II (Wizinowich et al. 2000) and Adams et al. (2013) using MMT/Aries.

The optical wavelength region probed with our GTC/OSIRIS transit observations fully overlaps with the $r^{\prime}$ and $i^{\prime}$ bands. We folded our data with the respective filter curves and found the results $\left(c_{r^{\prime}}=0.0023(7), c_{i^{\prime}}=0.0064(8)\right)$ to be consistent with the study of Zhao et al. $\left(2014, c_{\text {Zhao }, r^{\prime}}=0.003(1), c_{\text {Zhao }, i^{\prime}}=\right.$ $0.006(2))$.

Since the WHT/LIRIS filter curves for the near-infrared broadband filters $J, H$, and $K_{\mathrm{S}}$ differ slightly from those used by the Keck-II/NIRC 2 facility, an exact comparison between the respective measurements is not feasible. When neglecting these small differences in filter transmission, we found, however, that our results are consistent within $1 \sigma$ with the near-infrared values derived by all three studies, i.e., Zhao et al. (2014), Ngo et al. (2015), and Adams et al. (2013). Both Zhao et al. (2014) and Ngo et al. (2015) independently analyzed the Keck-II/NIRC2 data obtained in the $H$ and $K_{\mathrm{S}}$ band passes (while the $J$ and $K^{\prime}$ band data was only analyzed by $\mathrm{Ngo}$ et al. 2015) and arrived at different results, which are only consistent with each other within $2 \sigma$.

\section{A.4. Comparison to theoretical models}

Using their broadband measurements, Zhao et al. (2014) and Ngo et al. (2015) both determined physical properties of HATP-32B and arrived at similar results with effective temperatures around $T_{\text {eff }}=3550 \mathrm{~K}$. The exact results are listed in Table A.2.

Both studies made use of the PHOENIX stellar atmosphere models but relied on slightly different approaches, stellar parameters for HAT-P-32A, and different fixed values for the metallicity.

We conducted our own analysis by fitting a theoretical model of the form

$\mathcal{R}=k \cdot \frac{M_{\mathrm{HAT}-\mathrm{P}-32 \mathrm{~B}}\left(\lambda, T_{\mathrm{eff}}, \log g,[\mathrm{Fe} / \mathrm{H}]\right)}{M_{\mathrm{HAT}-\mathrm{P}-32 \mathrm{~A}}\left(\lambda, T_{\mathrm{eff}}, \log g,[\mathrm{Fe} / \mathrm{H}]\right)}$

to our optical and near-infrared data and the literature broadband measurements, excluding the upper limit Zhao et al. (2014) give for the $g^{\prime}$ band and the $K_{\mathrm{S}}$ band data point of Adams et al. (2013) for which no uncertainties were given. In Eq. (A.2) $k$ is a scaling factor (corresponding to the squared radius ratio of the two stars $\left.\left(R_{\mathrm{B}} / R_{\mathrm{A}}\right)^{2}\right)$ and $M_{\mathrm{HAT}-\mathrm{P}-32 \mathrm{~B}}$ and $M_{\mathrm{HAT}-\mathrm{P}-32 \mathrm{~A}}$ are PHOENIX stellar models, interpolated to specific stellar parameters from the model grid provided by Husser et al. (2013) with trilinear interpolation. During the fit the stellar parameters of HAT-P-32A were allowed to vary within the uncertainties of the given literature values $([\mathrm{Fe} / \mathrm{H}]=-0.04 \pm 0.08, \log g(\mathrm{cgs})=4.33 \pm 0.01$, Hartman et al. 2011, and $T_{\text {eff }}=6269 \pm 64 \mathrm{~K}$, Zhao et al. 2014) and an identical metallicity for both stars was enforced. For the comparison with the broadband points, we folded the PHOENIX model with the respective filter curves. In this step we used the exact instrument specific filter curve (downloaded from the facility web-pages) for each broadband data point. For the optical spectrum, we folded the PHOENIX model spectrum with a Gauss function to reduce the resolution to match the data.

We found that if we only fit the broadband measurements we arrive at similar values for the stellar parameters of HAT-P32B as those derived by Zhao et al. (2014) and Ngo et al. (2015). When including the optical data, however, the fit clearly favored cooler temperatures. Owing to the inconsistencies in the literature regarding the results obtained for the $H$ and $K_{\mathrm{S}}$ bands with 


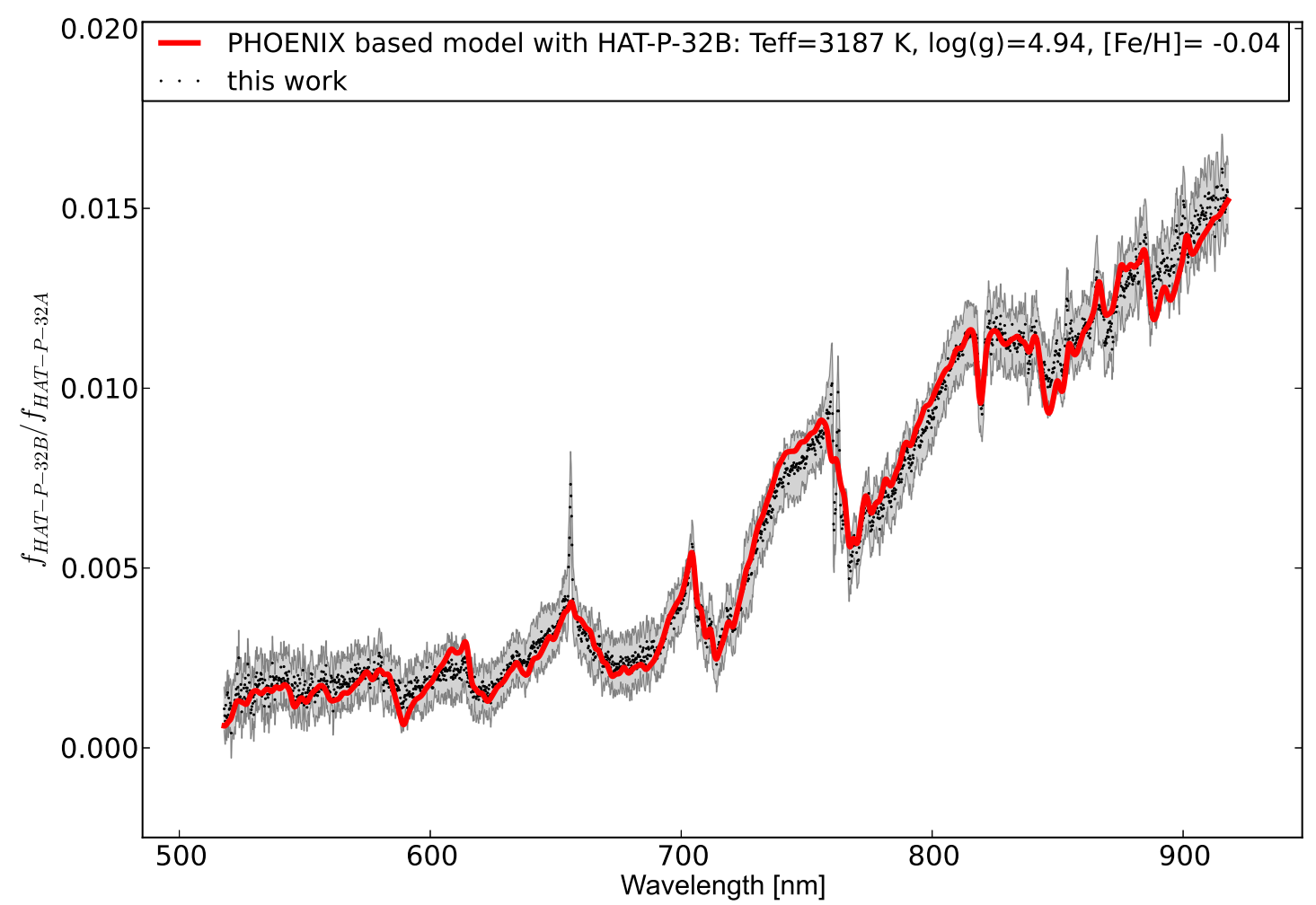

Fig. A.3. Relative optical spectrum HAT-P-32B/HAT-P-32A. The data is plotted as points, the $1 \sigma$ uncertainty intervals are given as a light gray shaded area. The best-fit PHOENIX model relative spectrum is given in red and relies on fixing the stellar properties of HAT-P-32A on literature values.

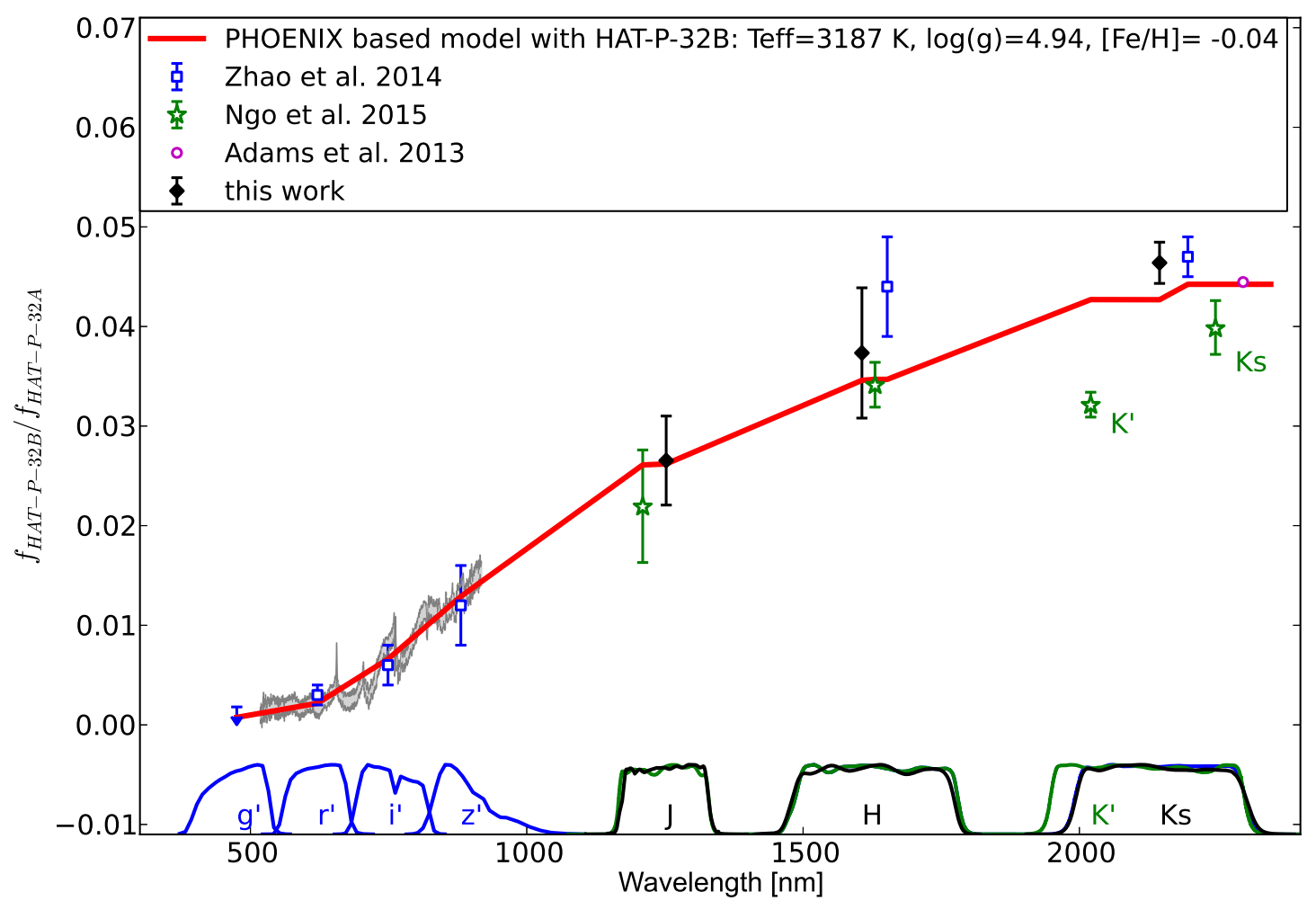

Fig. A.4. Relative optical and infrared photometry HAT-P-32B/HAT-P-32A. The results of the tree different literature studies are plotted together with our own $J, H$, and $K_{\mathrm{S}}$ band measurements. The optical relative spectrum from Fig. A.3 is also shown in light gray to emphasize the good agreement between this result and the literature photometry. The best-fit PHOENIX model relative spectrum (same as in Fig. A.3) evaluated in the same band passes as the observations is plotted in red. The points obtained in the same or very similar band passes are plotted at slightly shifted abscissa values from the actual center of the respective band pass to increase clarity of the figure. 
Table A.2. Comparison of derived stellar parameters for HAT-P-32B.

\begin{tabular}{ccccc}
\hline \hline Source & Date & $T_{\text {eff }}(\mathrm{K})$ & $\log g(\mathrm{cgs})$ & {$[\mathrm{Fe} / \mathrm{H}]$ (fixed/varied within prior) } \\
\hline This work & 2012-Sep-30 & $3187_{-71}^{+60}$ & $4.94_{-0.68}^{+0.50}$ & $-0.04_{-0.08}^{+0.08}$ \\
Zhao et al. (2014) & 2013-Mar-02 & $3565 \pm 82$ & $\ldots$ & -0.04 \\
Ngo et al. (2015) & 2012-Feb-02 & $3516 \pm 12$ & $4.8930 \pm 0.0098$ & 0.00 \\
Ngo et al. (2015) & 2013-Mar-02 & $3551 \pm 10$ & $4.8677 \pm 0.0070$ & 0.00 \\
\hline
\end{tabular}

Keck-II/NIRC2, we decided to include only our own data in the final optimization. The difference in the best-fit effective temperature due to this exclusion of broadband points is insignificant $(6 \mathrm{~K})$. Our results for the stellar parameters of HAT-P-32B are given in Table A.2. The uncertainties of these results were inflated with the red noise factor $\beta$, which was calculated as described in Sect. 3.2. The errors do not incorporate any intrinsic uncertainties of the PHOENIX stellar atmosphere models.

The best-fit model relative spectrum is plotted together with all data points (including those that were not regarded in the fit) in Figs. A.3 and A.4.
It stands out that the $K^{\prime}$ band result of Ngo et al. (2015) diverges significantly from all $K_{\mathrm{S}}$ band values including their own. This offset is too large to be explained by the difference between the $K^{\prime}$ and $K_{\mathrm{S}}$ band passes. Since the $K_{\mathrm{S}}$ and $K^{\prime}$ observations by $\mathrm{Ngo}$ et al. (2015) were conducted at different dates (13 month apart), a more likely explanation for this inconsistency could be stellar variability of HAT-P-32B due to activity, i.e., star spots or flares. We measure a prominent $H_{\alpha}$ emission line at $656 \mathrm{~nm}$ in the optical spectrum (see Fig. A.3), which is indicative of such stellar activity. 


\subsection{Conclusion of the characterization of the M-dwarf companion HAT-P-32B}

We extracted an optical spectrum of the M-dwarf companion HAT-P-32B and obtained infrared broadband photometry in the $J, H$, and $K_{S}$ bands.

Comparing the data with PHOENIX stellar atmosphere models we derived constraints for the metallicity, surface gravity and effective temperature of the star. While our results for the metallicity and surface gravity agree with the literature our value for the effective temperature of $T_{\text {eff }}=3187_{-71}^{+60}$ is significantly cooler than the results reported by Zhao et al. (2014) and Ngo et al. (2015), whose analysis is based solely on broadband infrared data. As their studies also relied on PHOENIX stellar atmosphere models to derive the stellar parameters these discrepancies cannot be attributed to inherent differences in the used reference models. We further found that when we artificially reduced our resolution to resemble typical broad bands and repeated the analysis, we arrived at comparable temperatures to the two previous studies. This leads us to conclude that the main cause for the divergence between the resulting temperatures is the higher resolution available in this study, providing us with the opportunity to compare the precise shape of atomic and molecular absorption features (e.g. TiO, VO, CaH, Na II) to the PHOENIX model predictions. 



\section{Chapter 4}

\section{Summary, discussion and outlook}

We observed the transmission spectra of two hot Jupiter planets from the ground at two different facilities, namely WASP-17b at VLT/FORS2 and HAT-P-32Ab at GTC/OSIRIS. The two instruments used are mounted at telescopes of the $10 \mathrm{~m}$ class and were chosen to yield the maximal available photon signal. The large telescope mirror size made both facility-instrument combinations attractive for exoplanet transmission studies before. FORS2 was in fact the first instrument used to study a transmission spectrum from the ground with multi-object broad-slit transit spectroscopy (Bean et al. 2010). Studies of exoplanet atmospheres with GTC/OSIRIS made use of both its tunable filter (e.g. Sing et al. 2011a, Murgas et al. 2012) and long-slit spectroscopy capabilities (Sing et al. 2012, Murgas et al. 2014, Mallonn et al. 2015). We, however, found that, when using these instruments, systematic noise signals, rather than photon noise, emerged as the limiting factor for the final reachable precision. These systematics had to be thoroughly studied and corrected for in order to derive useful results for the planetary transmission spectra.

\subsection{Transmission spectra}

Both observed transmission spectra do not exhibit the typical features predicted by most theoretical models, i.e. either strong alkali metal lines (for cooler temperatures) or a forest of many $\mathrm{TiO} / \mathrm{VO}$ absorption lines presenting as broad bands at lower resolution (for hotter temperatures). For HAT-P32Ab we instead found a very flat transmission spectrum. This result is in good agreement with previously published data by Gibson et al. (2013b) leading us to conclude that our correction of the systematic noise in the data was sufficient and the results are reliable. Featureless atmospheres have also been found for other exoplanets (e.g., Gibson et al., 2013a (WASP-29b); Knutson et al., 2014 (GJ 436b)) including the comprehensively studied super-Earth GJ 1214b (e.g. Bean et al. 2010, 2011, Berta et al. 2012, de Mooij et al. 2013, Kreidberg et al. 2014). In our case possible physical explanations are either a lower than expected atmospheric scale height or a high altitude could layer, masking all atmospheric features below. Future observations in the mid-infrared, where cloud induced scattering processes should decrease, could help to distinguish between these two possible scenarios.

In case of WASP-17b we could not find a convincing physical explanation for the 
strong increase in measured planet radii towards the blue. Since no spectroscopic transmission data for WASP-17b overlapping the wavelength region probed by us has been published, we could not independently verify the reliability of our results like it possible in the case of HAT-P-32Ab. When setting our results into context with existing literature, however, we found that our results could potentially act as a connection between the near-infrared HST/WFC3 measurements (Mandell et al. 2013) and broadband photometry observations taken in the optical (Bento et al. 2014), which are separated by a gap in transit depth slightly larger than $0.2 \%$. We believe that the comprehension of this planet's atmosphere could benefit from an independent repetition of our measurements with a de-coated LADC at FORS2 or a completely independent instrument and additional spectroscopy of the blue optical, where currently only broadband information is available.

\subsection{Instrument systematics}

As stated before, we found that the data obtained with both instruments was heavily affected by systematic noise signals of non-astrophysical origin. Such systematic noise is not uncommon and has been reported for space based observation with Spitzer and HST as well as other ground-based observatories (e.g. Berta et al. 2012, Stevenson et al. 2014b, Parviainen et al. 2015). In some cases new calibration steps were advised after thorough investigation of these systematics (e.g. Ballard et al. 2010, Stevenson et al. 2012), but in many cases the origin of the noise is not well understood.

Many instruments have exhibited significant systematics in some, but not all datasets. Rather than only using seemingly unaffected datasets and discarding the heavily affected ones, we believe it important to use the later to study the systematic noise signals and get a better understanding of their source and nature.

An immediate benefit of such investigations is that the gained knowledge can be incorporated in the correction of the systematics, opposed to either guessing the behavior of the systematic noise or employing methods which assume no prior knowledge of the systematics.

Another advantage of gaining a better understanding of the systematics is that it can be used to reduce or even completely avoid their impact on future observations. In some cases this could be realized by making small adjustments to the observing set up. For both OSIRIS and FORS2 (while the old LADC was still in place) one measure to reduce systematics would have been choosing a very close reference star, and placing both stars close to the center of rotation. The systematics would have been further reduced if the observations were conducted under small and slow changes of rotation angle. In reality a very close reference star is not always available and a full transit of the desired target can often not be observed without going through large changes in the rotation angle. For both instruments, however, also steps independent from the target of choice could be taken. In FORS2 observations, prior to the LADC exchange, the LADC prism distance could be fixed to zero during the whole observation to avoid the jumps associated with a restart of an Observing Block (which themselves cannot be avoided with certainty as small problems could 
occur at any time during the observations). This measure was taken by us to avoid flux jumps for FORS2 observations of the hot Jupiters WASP-31b, WASP-39b and Wasp-19b in 2012 and resulted in the desired outcome.

Similarly, action was taken for OSIRIS to improve the light curve quality by increasing the slit width from $12^{\prime \prime}$ to $40^{\prime \prime}$, yielding a significant reduction of the seeing dependent systematics (e.g. Parviainen et al. 2016).

Last but not least, the knowledge gained about the systematic noise exhibited by a certain instrument can be of use when defining the requirements of the next generation of instruments dedicated to exoplanet atmosphere characterization. Based on the systematics found in this work, our main advice would be to make an effort in avoiding any elements that are not co-rotating with the rest of the optical set up. If this proofs to be impossible, very high demands on the homogeneity of any differentially rotating elements should be made.

\subsection{Outlook on future observations with VLT/FORS2 and GTC/OSIRIS}

Following the initial success of Bean et al. (2010, 2011) FOSR2 had been a popular choice for planned transit spectroscopy studies. Since no publications came forth, it is reasonable to assume that most observations granted for this purpose were affected by similar systematics like the one we discuss in Chapter 2. After the European Southern Observatory recently responded to this situation by exchanging the LADC prisms, FORS2 now appears to be ready to be used routinely for exoplanet transit spectroscopy studies (Boffin et al. 2015, Sedaghati et al. 2015). The planet WASP-19b used in their test, however, has a very short transit duration and can be observed under little change of paralactic angle. While this makes it an excellent target for a first assessment, it is not perfectly suited to confirm fully regained capabilities of the instrument. A more convincing verification would be the reobservation of a target like WASP-17b, which exhibits a long transit and can only be observed fully when passing the meridian during the observation. Until such a test is performed yielding systematic noise free data, the future of FORS2 is still questionable. In case all sources of systematic truly have been disposed of by exchanging the LADC, the instrument could once again find itself in high demand from the exoplanet community and finally become the efficient and reliable tool for ground-based transmission spectroscopy studies that it was initially assumed to be. The wavelength independent systematics in GTC/OSIRIS, associated with pupil vignetting, will remain an issue for many set ups, owing to a limited availability of targets with short transits and close by reference stars. These instrument signals, however, only affect the ability to derive results for the white light curve and have little influence on the transmission spectrum. Therefore, the problem can easily be overcome if reliable literature data for the target exists. Alternatively photometric observations could be scheduled at a different telescope, preferably observing the exact same transit event and using a broadband filter whose coverage is fully included in the wavelength range studied with OSIRIS.

Another development for GTC is that its dome shutter, which in the past could not 
be fully opened and was limiting the observable elevation to a little under $80^{\circ}$ over the horizon, is in the process of being fixed ${ }^{1}$. With a fully opening roof previously inaccessible promising targets will be observable with GTC/OSIRIS. Furthermore, EMIR, a wide-field camera and a near-infrared multi-object medium-resolution spectrograph, is planned to be mounted at GTC in the foreseeable future. With a wavelength coverage of $0.9-2.5 \mu \mathrm{m}$ it would provide the means to expand the GTC exoplanet survey to the investigation of the broad molecular absorption bands of e.g. water and methane in the infrared transmission spectra and the measurement reflection spectra during secondary eclipse.

\footnotetext{
${ }^{1}$ status as of September 2015, private communication with GTC staff
} 


\section{Bibliography}

Adams, E. R., Dupree, A. K., Kulesa, C. \& McCarthy, D. (2013) Adaptive Optics Images. II. 12 Kepler Objects of Interest and 15 Confirmed Transiting Planets. AJ, 146, 9. 2.6.3

Adams, E. R., Seager, S. \& Elkins-Tanton, L. (2008) Ocean Planet or Thick Atmosphere: On the Mass-Radius Relationship for Solid Exoplanets with Massive Atmospheres. ApJ, 673, 1160-1164. 1.2

Alonso, R., Guillot, T., Mazeh, T., Aigrain, S., Alapini, A., Barge, P., Hatzes, A. \& Pont, F. (2009) The secondary eclipse of the transiting exoplanet CoRoT-2b. $A \& A$, 501, L23-L26. 1.2.1

Anderson, D. R., Hellier, C., Gillon, M., Triaud, A. H. M. J., Smalley, B., Hebb, L., Collier Cameron, A., Maxted, P. F. L. et al. (2010) Wasp-17b: An Ultra-Low Density Planet in a Probable Retrograde Orbit. ApJ, 709, 159-167. 2.1, 2.5.5, 2.3, 2.5.7, 2.6, 2.4, 2.20, 2.21, 2.22, 2.23

Appenzeller, I., Fricke, K., Fürtig, W., Gässler, W., Häfner, R., Harke, R., Hess, H.-J., Hummel, W. et al. (1998) Successful commissioning of FORS1 - the first optical instrument on the VLT. The Messenger, 94, 1-6. 2.2

Asplund, M., Grevesse, N., Sauval, A. J. \& Scott, P. (2009) The Chemical Composition of the Sun. ARAESA, 47, 481-522. 2.6

Avila, G., Rupprecht, G. \& Beckers, J. M. (1997) Atmospheric dispersion correction for the FORS Focal Reducers at the ESO VLT. In Society of Photo-Optical Instrumentation Engineers (SPIE) Conference Series, edited by A. L. Ardeberg, vol. 2871 of Society of Photo-Optical Instrumentation Engineers (SPIE) Conference Series. 2.4

Ballard, S., Charbonneau, D., Deming, D., Knutson, H. A., Christiansen, J. L., Holman, M. J., Fabrycky, D., Seager, S. et al. (2010) A Search for a Sub-EarthSized Companion to GJ 436 and a Novel Method to Calibrate Warm Spitzer IRAC Observations. PASP, 122, 1341-1352. 4.2

Ballard, S., Fabrycky, D., Fressin, F., Charbonneau, D., Desert, J.-M., Torres, G., Marcy, G., Burke, C. J. et al. (2011) The Kepler-19 System: A Transiting 2.2 R $\oplus$ Planet and a Second Planet Detected via Transit Timing Variations. ApJ, 743, 200. 1.1.2.1 
Ballerini, P., Micela, G., Lanza, A. F. \& Pagano, I. (2012) Multiwavelength flux variations induced by stellar magnetic activity: effects on planetary transits. $A \mathscr{E} A$, 539, A140. 2.6.2

Barclay, T., Rowe, J. F., Lissauer, J. J., Huber, D., Fressin, F., Howell, S. B., Bryson, S. T., Chaplin, W. J. et al. (2013) A sub-Mercury-sized exoplanet. Nature, 494, 452-454. 1.1.2

Barman, T. S., Hauschildt, P. H. \& Allard, F. (2005) Phase-Dependent Properties of Extrasolar Planet Atmospheres. ApJ, 632, 1132-1139. 2.6

Barman, T. S., Hauschildt, P. H., Schweitzer, A., Stancil, P. C., Baron, E. \& Allard, F. (2003) NLTE Calculations of a Na D Doublet in the Atmosphere of the Transiting Planet HD209458b. In Scientific Frontiers in Research on Extrasolar Planets, edited by D. Deming \& S. Seager, vol. 294 of Astronomical Society of the Pacific Conference Series. 2.6

Barman, T. S., Konopacky, Q. M., Macintosh, B. \& Marois, C. (2015) Simultaneous Detection of Water, Methane, and Carbon Monoxide in the Atmosphere of Exoplanet HR8799b. ApJ, 804, 61. 1.2.1

Bean, J. L., Désert, J.-M., Kabath, P., Stalder, B., Seager, S., Miller-Ricci Kempton, E., Berta, Z. K., Homeier, D. et al. (2011) The Optical and Near-infrared Transmission Spectrum of the Super-Earth GJ 1214b: Further Evidence for a Metal-rich Atmosphere. ApJ, 743, 92. 1.3, 2.4.1, 2.4.2, 2.7, 4.1, 4.3

Bean, J. L., Miller-Ricci Kempton, E. \& Homeier, D. (2010) A ground-based transmission spectrum of the super-Earth exoplanet GJ 1214b. Nature, 468, 669-672. $1.2 .2 .2,1.3,2.4 .1,2.4 .2,2.9,2.7,4,4.1,4.3$

Benedict, G. F., McArthur, B. E., Forveille, T., Delfosse, X., Nelan, E., Butler, R. P., Spiesman, W., Marcy, G. et al. (2002) A Mass for the Extrasolar Planet Gliese 876b Determined from Hubble Space Telescope Fine Guidance Sensor 3 Astrometry and High-Precision Radial Velocities. ApJ, 581, L115-L118. 1.1.1

Benedict, G. F., McArthur, B. E., Gatewood, G., Nelan, E., Cochran, W. D., Hatzes, A., Endl, M., Wittenmyer, R. et al. (2006) The Extrasolar Planet $\epsilon$ Eridani b: Orbit and Mass. AJ, 132, 2206-2218. 1.1.1

Bento, J., Wheatley, P. J., Copperwheat, C. M., Fortney, J. J., Dhillon, V. S., Hickman, R., Littlefair, S. P., Marsh, T. R. et al. (2014) Optical transmission photometry of the highly inflated exoplanet WASP-17b. MNRAS, 437, 15111518. 1.2.2.2, 1.4, 2.1, 2.5.5, 2.3, 2.6, 2.20, 2.21, 2.22, 2.23, 2.7, 4.1

Bergfors, C., Brandner, W., Daemgen, S., Biller, B., Hippler, S., Janson, M., Kudryavtseva, N., Geißler, K. et al. (2013) Stellar companions to exoplanet host stars: Lucky Imaging of transiting planet hosts. MNRAS, 428, 182-189. 2.6.3 
Berta, Z. K., Charbonneau, D., Désert, J.-M., Miller-Ricci Kempton, E., McCullough, P. R., Burke, C. J., Fortney, J. J., Irwin, J. et al. (2012) The Flat Transmission Spectrum of the Super-Earth GJ1214b from Wide Field Camera 3 on the Hubble Space Telescope. ApJ, 747, 35. 1.2.2.1, 1.3, 2.5.3, 4.1, 4.2

Boffin, H., Blanchard, G., Gonzalez, O., Moehler, S., Sedaghati, E., Gibson, N., van den Ancker, M., Smoker, J. et al. (2015) Making FORS2 Fit for Exoplanet Observations (again). The Messenger, 159, 6-9. 2.4.2, 2.7, 4.3

Brogi, M., Snellen, I. A. G., de Kok, R. J., Albrecht, S., Birkby, J. \& de Mooij, E. J. W. (2012) The signature of orbital motion from the dayside of the planet $\tau$ Boötis b. Nature, 486, 502-504. 1.2.1

Burrows, A., Rauscher, E., Spiegel, D. S. \& Menou, K. (2010) Photometric and Spectral Signatures of Three-dimensional Models of Transiting Giant Exoplanets. ApJ, 719, 341-350. 1.3

Carter, J. A. \& Winn, J. N. (2009) Parameter Estimation from Time-series Data with Correlated Errors: A Wavelet-based Method and its Application to Transit Light Curves. ApJ, 704, 51-67. 2.5.4

Charbonneau, D., Berta, Z. K., Irwin, J., Burke, C. J., Nutzman, P., Buchhave, L. A., Lovis, C., Bonfils, X. et al. (2009) A super-Earth transiting a nearby lowmass star. Nature, 462, 891-894. 1.2.2

Charbonneau, D., Brown, T. M., Noyes, R. W. \& Gilliland, R. L. (2002) Detection of an Extrasolar Planet Atmosphere. ApJ, 568, 377-384. 1.2.2.1

Charbonneau, D., Knutson, H. A., Barman, T., Allen, L. E., Mayor, M., Megeath, S. T., Queloz, D. \& Udry, S. (2008) The Broadband Infrared Emission Spectrum of the Exoplanet HD 189733b. ApJ, 686, 1341-1348. 1.2.2.1

Coccato, L., Bramich, D. M., Freudling, W. \& Moehler, S. (2014) Removal of systematics in photometric measurements: static and rotating illumination corrections in FORS2@VLT data. MNRAS, 438, 1256-1266. 2.4

Croll, B., Lafreniere, D., Albert, L., Jayawardhana, R., Fortney, J. J. \& Murray, N. (2011) Near-infrared Thermal Emission from WASP-12b: Detections of the Secondary Eclipse in Ks, H, and J. AJ, 141, 30. 1.2.1

Crossfield, I. J. M., Barman, T., Hansen, B. M. S., Tanaka, I. \& Kodama, T. (2012) Re-evaluating WASP-12b: Strong Emission at $2.315 \mu \mathrm{m}$, Deeper Occultations, and an Isothermal Atmosphere. ApJ, 760, 140. 2.6.3

Cumming, A., Marcy, G. W. \& Butler, R. P. (1999) The Lick Planet Search: Detectability and Mass Thresholds. ApJ, 526, 890-915. 1.1.1

de Mooij, E. J. W., Brogi, M., de Kok, R. J., Snellen, I. A. G., Croll, B., Jayawardhana, R., Hoekstra, H., Otten, G. P. P. L. et al. (2013) Search for Rayleigh Scattering in the Atmosphere of GJ1214b. ApJ, 771, 109. 1.3, 4.1 
Deming, D., Wilkins, A., McCullough, P., Burrows, A., Fortney, J. J., Agol, E., Dobbs-Dixon, I., Madhusudhan, N. et al. (2013) Infrared Transmission Spectroscopy of the Exoplanets HD 209458b and XO-1b Using the Wide Field Camera3 on the Hubble Space Telescope. ApJ, 774, 95. 1.3

Désert, J.-M., Sing, D., Vidal-Madjar, A., Hébrard, G., Ehrenreich, D., Lecavelier Des Etangs, A., Parmentier, V., Ferlet, R. et al. (2011) Transit spectrophotometry of the exoplanet HD 189733b. II. New Spitzer observations at $3.6 \mu \mathrm{m}$. A $\& A, \mathbf{5 2 6}$, A12. 2.6.2

Désert, J.-M., Vidal-Madjar, A., Lecavelier Des Etangs, A., Sing, D., Ehrenreich, D., Hébrard, G. \& Ferlet, R. (2008) TiO and VO broad band absorption features in the optical spectrum of the atmosphere of the hot-Jupiter $<$ ASTROBJ $>$ HD 209458b </ASTROBJ >. A\& A, 492, 585-592. 1.3

Diamond-Lowe, H., Stevenson, K. B., Bean, J. L., Line, M. R. \& Fortney, J. J. (2014) New Analysis Indicates No Thermal Inversion in the Atmosphere of HD 209458b. ApJ, 796, 66. 1.2.2.1, 1.3

Díaz, R. F., Almenara, J. M., Santerne, A., Moutou, C., Lethuillier, A. \& Deleuil, M. (2014) PASTIS: Bayesian extrasolar planet validation - I. General framework, models, and performance. MNRAS, 441, 983-1004. 1.1.2

Dragomir, D. (2015) The variability of nearby exoplanet host stars. IAU General Assembly, 22, 57688. 1.1.2.3

Eastman, J., Gaudi, B. S. \& Agol, E. (2013) EXOFAST: A Fast Exoplanetary Fitting Suite in IDL. PASP, 125, 83-112. 2.5

Eastman, J., Siverd, R. \& Gaudi, B. S. (2010) Achieving Better Than 1 Minute Accuracy in the Heliocentric and Barycentric Julian Dates. PASP, 122, 935-946. 2.3.3

Foreman-Mackey, D., Hogg, D. W., Lang, D. \& Goodman, J. (2013) emcee: The MCMC Hammer. PASP, 125, 306-312. 2.5.3

Fortney, J. J., Lodders, K., Marley, M. S. \& Freedman, R. S. (2008) A Unified Theory for the Atmospheres of the Hot and Very Hot Jupiters: Two Classes of Irradiated Atmospheres. ApJ, 678, 1419-1435. 1.3, 2.6

Fortney, J. J., Shabram, M., Showman, A. P., Lian, Y., Freedman, R. S., Marley, M. S. \& Lewis, N. K. (2010) Transmission Spectra of Three-Dimensional Hot Jupiter Model Atmospheres. ApJ, 709, 1396-1406. 1.3

Freedman, R. S., Marley, M. S. \& Lodders, K. (2008) Line and Mean Opacities for Ultracool Dwarfs and Extrasolar Planets. ApJS, 174, 504-513. 1.3

Fressin, F., Knutson, H. A., Charbonneau, D., O’Donovan, F. T., Burrows, A., Deming, D., Mandushev, G. \& Spiegel, D. (2010) The Broadband Infrared Emission Spectrum of the Exoplanet TrES-3. ApJ, 711, 374-379. 1.2.1, 1.3 
Gibson, N. P. (2014) Reliable inference of exoplanet light-curve parameters using deterministic and stochastic systematics models. MNRAS, 445, 3401-3414. 1.4

Gibson, N. P., Aigrain, S., Barstow, J. K., Evans, T. M., Fletcher, L. N. \& Irwin, P. G. J. (2013a) A Gemini ground-based transmission spectrum of WASP-29b: a featureless spectrum from 515 to $720 \mathrm{~nm}$. MNRAS, 428, 3680-3692. 1.2.2.2, 1.3, 4.1

Gibson, N. P., Aigrain, S., Barstow, J. K., Evans, T. M., Fletcher, L. N. \& Irwin, P. G. J. (2013b) The optical transmission spectrum of the hot Jupiter HAT-P-32b: clouds explain the absence of broad spectral features? MNRAS, 436, 2974-2988. $1.2 .2 .2,1.3,1.4,4.1$

Gibson, N. P., Aigrain, S., Roberts, S., Evans, T. M., Osborne, M. \& Pont, F. (2012) A Gaussian process framework for modelling instrumental systematics: application to transmission spectroscopy. MNRAS, 419, 2683-2694. 1.4

Gibson, N. P., Pont, F. \& Aigrain, S. (2011) A new look at NICMOS transmission spectroscopy of HD 189733, GJ-436 and XO-1: no conclusive evidence for molecular features. MNRAS, 411, 2199-2213. 1.2.2.1

Gillon, M., Lanotte, A. A., Barman, T., Miller, N., Demory, B.-O., Deleuil, M., Montalbán, J., Bouchy, F. et al. (2010) The thermal emission of the young and massive planet CoRoT-2b at 4.5 and $8 \mu \mathrm{m}$. A $\& A, \mathbf{5 1 1}, \mathrm{A} 3.1 .3$

Holman, M. J., Fabrycky, D. C., Ragozzine, D., Ford, E. B., Steffen, J. H., Welsh, W. F., Lissauer, J. J., Latham, D. W. et al. (2010) Kepler-9: A System of Multiple Planets Transiting a Sun-Like Star, Confirmed by Timing Variations. Science, 330, 51-. 1.1.2.1

Horne, K. (1986) An optimal extraction algorithm for CCD spectroscopy. PASP, 98, 609-617. 2.3

Husser, T.-O., Wende-von Berg, S., Dreizler, S., Homeier, D., Reiners, A., Barman, T. \& Hauschildt, P. H. (2013) A new extensive library of PHOENIX stellar atmospheres and synthetic spectra. $A \mathscr{G} A, \mathbf{5 5 3}$, A6. 2.5.1

Jordán, A., Espinoza, N., Rabus, M., Eyheramendy, S., Sing, D. K., Désert, J.-M., Bakos, G. Á., Fortney, J. J. et al. (2013) A Ground-based Optical Transmission Spectrum of WASP-6b. ApJ, 778, 184. 1.2.2.2, 1.3

Kashyap, V. L. \& Drake, J. J. (2000) PINTofALE: Package for the Interactive Analysis of Line Emission. In AAS/High Energy Astrophysics Division \#5, vol. 32 of Bulletin of the American Astronomical Society. 2.3 .2

Kipping, D. M. (2009) Transit timing effects due to an exomoon. MNRAS, 392, 181-189. 1.1.2.1

Knutson, H. A., Benneke, B., Deming, D. \& Homeier, D. (2014) A featureless transmission spectrum for the Neptune-mass exoplanet GJ436b. Nature, 505, 66-68. 1.3, 4.1 
Knutson, H. A., Charbonneau, D., Allen, L. E., Burrows, A. \& Megeath, S. T. (2008) The 3.6-8.0 $\mu \mathrm{m}$ Broadband Emission Spectrum of HD 209458b: Evidence for an Atmospheric Temperature Inversion. ApJ, 673, 526-531. 1.2.1, 1.2.2.1, 1.3

Knutson, H. A., Charbonneau, D., Allen, L. E., Fortney, J. J., Agol, E., Cowan, N. B., Showman, A. P., Cooper, C. S. et al. (2007) A map of the day-night contrast of the extrasolar planet HD 189733b. Nature, 447, 183-186. 1.2.1

Knutson, H. A., Charbonneau, D., Burrows, A., O’Donovan, F. T. \& Mandushev, G. (2009) Detection of A Temperature Inversion in the Broadband Infrared Emission Spectrum of TrES-4. ApJ, 691, 866-874. 1.2.1

Knutson, H. A., Howard, A. W. \& Isaacson, H. (2010) A Correlation Between Stellar Activity and Hot Jupiter Emission Spectra. ApJ, 720, 1569-1576. 1.3

Koldovský, Z., Tichavský, P. \& Oja, E. (2006) Efficient Variant of Algorithm FastICA for Independent Component Analysis Attaining the Cramer-Rao Lower Bound. IEEE Transactions on Neural Networks, 17, 1265-1277. 2.5.6

Kopal, Z. (1950) Detailed effects of limb darkening upon light and velocity curves of close binary systems. Harvard College Observatory Circular, 454, 1-12. 1.1.2.2

Kreidberg, L., Bean, J. L., Désert, J.-M., Benneke, B., Deming, D., Stevenson, K. B., Seager, S., Berta-Thompson, Z. et al. (2014) Clouds in the atmosphere of the super-Earth exoplanet GJ1214b. Nature, 505, 69-72. 1.3, 2.5.6, 4.1

Lecavelier Des Etangs, A., Pont, F., Vidal-Madjar, A. \& Sing, D. (2008) Rayleigh scattering in the transit spectrum of HD 189733b. A\& A, 481, L83-L86. 2.6.1

Machalek, P., McCullough, P. R., Burke, C. J., Valenti, J. A., Burrows, A. \& Hora, J. L. (2008) Thermal Emission of Exoplanet XO-1b. ApJ, 684, 1427-1432. 1.3

Madhusudhan, N. (2012) C/O Ratio as a Dimension for Characterizing Exoplanetary Atmospheres. ApJ, 758, 36. 1.3

Madhusudhan, N. \& Seager, S. (2010) On the Inference of Thermal Inversions in Hot Jupiter Atmospheres. ApJ, 725, 261-274. 1.3

Mallonn, M., von Essen, C., Weingrill, J., Strassmeier, K. G., Ribas, I., Carroll, T. A., Herrero, E., Granzer, T. et al. (2015) Transmission spectroscopy of the inflated exo-Saturn HAT-P-19b. A\&A A, 580, A60. 1.2.2.2, 4

Mandel, K. \& Agol, E. (2002) Analytic Light Curves for Planetary Transit Searches. $A p J$, 580, L171-L175. 2.5, 2.5.2, 2.5.5

Mandell, A. M., Haynes, K., Sinukoff, E., Madhusudhan, N., Burrows, A. \& Deming, D. (2013) Exoplanet Transit Spectroscopy Using WFC3: WASP-12 b, WASP-17 b, and WASP-19 b. ApJ, 779, 128. 1.3, 1.4, 2.1, 2.5.5, 2.3, 2.5.6, 2.15, 2.5.7.1, $2.18,2.6,2.20,2.21,2.22,2.23,2.7,4.1$ 
Markwardt, C. B. (2009) Non-linear Least-squares Fitting in IDL with MPFIT. In Astronomical Data Analysis Software and Systems XVIII, edited by D. A. Bohlender, D. Durand \& P. Dowler, vol. 411 of Astronomical Society of the Pacific Conference Series. 2.5.2

Mayor, M. \& Queloz, D. (1995) A Jupiter-mass companion to a solar-type star. Nature, 378, 355-359. 1

McArthur, B. E., Endl, M., Cochran, W. D., Benedict, G. F., Fischer, D. A., Marcy, G. W., Butler, R. P., Naef, D. et al. (2004) Detection of a Neptune-Mass Planet in the $\rho^{1}$ Cancri System Using the Hobby-Eberly Telescope. ApJ, 614, L81-L84. 1.1 .1

Moehler, S., Freudling, W., Møller, P., Patat, F., Rupprecht, G. \& O’Brien, K. (2010) Correction of Field Rotator-Induced Flat-Field Systematics - A Case Study Using Archived VLT-FORS Data. PASP, 122, 93-102. 2.4, 2.4.2

Murgas, F., Pallé, E., Cabrera-Lavers, A., Colón, K. D., Martín, E. L. \& Parviainen, H. (2012) Narrow band H $\alpha$ photometry of the super-Earth GJ 1214b with GTC/OSIRIS tunable filters. A\&A, 544, A41. 1.2.2.2, 4

Murgas, F., Pallé, E., Zapatero Osorio, M. R., Nortmann, L., Hoyer, S. \& CabreraLavers, A. (2014) The GTC exoplanet transit spectroscopy survey . I. OSIRIS transmission spectroscopy of the short period planet WASP-43b. A\&A, 563, A41. 1.2.2.2, 4

Ngo, H., Knutson, H. A., Hinkley, S., Crepp, J. R., Bechter, E. B., Batygin, K., Howard, A. W., Johnson, J. A. et al. (2015) Friends of Hot Jupiters. II. No Correspondence between Hot-jupiter Spin-Orbit Misalignment and the Incidence of Directly Imaged Stellar Companions. ApJ, 800, 138. 2.6.3, 3.2

Nikolov, N., Sing, D. K., Burrows, A. S., Fortney, J. J., Henry, G. W., Pont, F., Ballester, G. E., Aigrain, S. et al. (2015) HST hot-Jupiter transmission spectral survey: haze in the atmosphere of WASP-6b. MNRAS, 447, 463-478. 1.2.2.1, 1.3

Nikolov, N., Sing, D. K., Pont, F., Burrows, A. S., Fortney, J. J., Ballester, G. E., Evans, T. M., Huitson, C. M. et al. (2014) Hubble Space Telescope hot Jupiter transmission spectral survey: a detection of $\mathrm{Na}$ and strong optical absorption in HAT-P-1b. MNRAS, 437, 46-66. 1.2.2.1, 1.3

Nortmann, L., Dreizler, S., Bean, J. L. \& Barman, T. (2015) Ground-based investigation of the highly irradiated atmosphere of the exoplanet WASP-17 b in the red optical with VLT/FORS2. Submitted for publication in A\&A. (document), 2

Nortmann, L., Pallé, E., Murgas, F., Dreizler, S., Iro, N. \& Cabrera-Lavers, A. (2016) The GTC exoplanet transit spectroscopy survey. IV. Confirmation of the flat transmission spectrum of HAT-P-32b. AEAA, 594, A65. (document), 3 
Ohta, Y., Taruya, A. \& Suto, Y. (2005) The Rossiter-McLaughlin Effect and Analytic Radial Velocity Curves for Transiting Extrasolar Planetary Systems. ApJ, 622, 1118-1135. 1.1.2.1

Oshagh, M., Santos, N. C., Ehrenreich, D., Haghighipour, N., Figueira, P., Santerne, A. \& Montalto, M. (2014) Impact of occultations of stellar active regions on transmission spectra. Can occultation of a plage mimic the signature of a blue sky? A\&A, 568, A99. 1.3

Parmentier, V., Showman, A. P. \& Lian, Y. (2013) 3D mixing in hot Jupiters atmospheres. I. Application to the day/night cold trap in HD 209458b. A\&GA, 558, A91. 1.3

Parviainen, H., Aigrain, S., Thatte, N., Barstow, J. K., Evans, T. M. \& Gibson, N. (2015) Exoplanet transmission spectroscopy using KMOS. MNRAS, 453, 38753885. 4.2

Parviainen, H., Pallé, E., Nortmann, L., Nowak, G., Iro, N., Murgas, F. \& Aigrain, S. (2016) The GTC exoplanet transit spectroscopy survey. II. An overly large Rayleigh-like feature for exoplanet TrES-3b. A\&A, 585, A114. 1.3, 4.2

Pont, F., Knutson, H., Gilliland, R. L., Moutou, C. \& Charbonneau, D. (2008) Detection of atmospheric haze on an extrasolar planet: the 0.55-1.05 $\mu \mathrm{m}$ transmission spectrum of HD 189733b with the HubbleSpaceTelescope. MNRAS, 385, 109-118. 2.6.1

Santerne, A., Díaz, R. F., Almenara, J.-M., Lethuillier, A., Deleuil, M. \& Moutou, C. (2013) Astrophysical false positives in exoplanet transit surveys: why do we need bright stars? In SF2A-2013: Proceedings of the Annual meeting of the French Society of Astronomy and Astrophysics, edited by L. Cambresy, F. Martins, E. Nuss \& A. Palacios. 1.1.2

Schwarz, H., Brogi, M., de Kok, R., Birkby, J. \& Snellen, I. (2015) Evidence against a strong thermal inversion in HD 209458b from high-dispersion spectroscopy. A\&A, 576, A111. 1.3

Seager, S. \& Deming, D. (2010) Exoplanet Atmospheres. ARA\&A, 48, 631-672. 1.2

Seager, S. \& Mallén-Ornelas, G. (2003) A Unique Solution of Planet and Star Parameters from an Extrasolar Planet Transit Light Curve. ApJ, 585, 1038-1055. 1.1 .2

Seager, S. \& Sasselov, D. D. (2000) Theoretical Transmission Spectra during Extrasolar Giant Planet Transits. ApJ, 537, 916-921. 1.2.2, 1.3

Sedaghati, E., Boffin, H. M. J., Csizmadia, S., Gibson, N., Kabath, P., Mallonn, M. \& Van den Ancker, M. E. (2015) Regaining the FORS: optical ground-based transmission spectroscopy of the exoplanet WASP-19b with VLT+FORS2. A $\dot{\varepsilon}$, 576, L11. 2.4.2, 2.7, 4.3 
Sing, D. K., Désert, J.-M., Fortney, J. J., Lecavelier Des Etangs, A., Ballester, G. E., Cepa, J., Ehrenreich, D., López-Morales, M. et al. (2011a) Gran Telescopio Canarias OSIRIS transiting exoplanet atmospheric survey: detection of potassium in XO-2b from narrowband spectrophotometry. A\&A, 527, A73. 1.2.2.2, 4

Sing, D. K., Huitson, C. M., Lopez-Morales, M., Pont, F., Désert, J.-M., Ehrenreich, D., Wilson, P. A., Ballester, G. E. et al. (2012) GTC OSIRIS transiting exoplanet atmospheric survey: detection of sodium in $\mathrm{XO}-2 \mathrm{~b}$ from differential long-slit spectroscopy. MNRAS, 426, 1663-1670. 1.2.2.2, 4

Sing, D. K., Pont, F., Aigrain, S., Charbonneau, D., Désert, J.-M., Gibson, N., Gilliland, R., Hayek, W. et al. (2011b) Hubble Space Telescope transmission spectroscopy of the exoplanet HD 189733b: high-altitude atmospheric haze in the optical and near-ultraviolet with STIS. MNRAS, 416, 1443-1455. 1.1.2.3, 1.3

Sing, D. K., Wakeford, H. R., Showman, A. P., Nikolov, N., Fortney, J. J., Burrows, A. S., Ballester, G. E., Deming, D. et al. (2015) HST hot-Jupiter transmission spectral survey: detection of potassium in WASP-31b along with a cloud deck and Rayleigh scattering. MNRAS, 446, 2428-2443. 1.2.2.1, 1.3

Snellen, I. A. G., de Mooij, E. J. W. \& Burrows, A. (2010) Bright optical day-side emission from extrasolar planet CoRoT-2b. A\&GA, 513, A76. 1.2.1

Southworth, J., Hinse, T. C., Dominik, M., Fang, X.-S., Harpsøe, K., Jørgensen, U. G., Kerins, E., Liebig, C. et al. (2012) High-precision photometry by telescope defocusing - IV. Confirmation of the huge radius of WASP-17 b. MNRAS, 426, 1338-1348. 2.1, 2.5, 2.5.5, 2.3, 2.6.1, 2.20, 2.21, 2.22, 2.23

Spiegel, D. S., Silverio, K. \& Burrows, A. (2009) Can TiO Explain Thermal Inversions in the Upper Atmospheres of Irradiated Giant Planets? ApJ, 699, 14871500. 1.3

Stevenson, K. B., Bean, J. L., Fabrycky, D. \& Kreidberg, L. (2014a) A Hubble Space Telescope Search for a Sub-Earth-sized Exoplanet in the GJ 436 System. ApJ, 796, 32. 1.3

Stevenson, K. B., Bean, J. L., Seifahrt, A., Désert, J.-M., Madhusudhan, N., Bergmann, M., Kreidberg, L. \& Homeier, D. (2014b) Transmission Spectroscopy of the Hot Jupiter WASP-12b from 0.7 to $5 \mu \mathrm{m}$. AJ, 147, 161. 2.5.6, 4.2

Stevenson, K. B., Désert, J.-M., Line, M. R., Bean, J. L., Fortney, J. J., Showman, A. P., Kataria, T., Kreidberg, L. et al. (2014c) Thermal structure of an exoplanet atmosphere from phase-resolved emission spectroscopy. Science, 346, 838-841. 1.2 .1

Stevenson, K. B., Harrington, J., Fortney, J. J., Loredo, T. J., Hardy, R. A., Nymeyer, S., Bowman, W. C., Cubillos, P. et al. (2012) Transit and Eclipse Analyses of the Exoplanet HD 149026b Using BLISS Mapping. ApJ, 754, 136. 4.2 
Thatte, A., Deroo, P. \& Swain, M. R. (2010) Selective principal component extraction and reconstruction: a novel method for ground based exoplanet spectroscopy. $A \mathscr{G} A, \mathbf{5 2 3}$, A35. 1.4, 2.5.6

Tichavský, P., Koldovský, Z., Yeredor, A., Gomez-Herrero, G. \& Doron, E. (2008) A Hybrid Technique for Blind Separation of Non-Gaussian and Time-Correlated Sources Using a Multicomponent Approach. IEEE Transactions on Neural Networks, 19, 421-430. 2.5.6

Todorov, K., Deming, D., Harrington, J., Stevenson, K. B., Bowman, W. C., Nymeyer, S., Fortney, J. J. \& Bakos, G. A. (2010) Spitzer IRAC Secondary Eclipse Photometry of the Transiting Extrasolar Planet HAT-P-1b. ApJ, 708, 498-504. 1.2.2.1

Wakeford, H. R., Sing, D. K., Deming, D., Gibson, N. P., Fortney, J. J., Burrows, A. S., Ballester, G., Nikolov, N. et al. (2013) HST hot Jupiter transmission spectral survey: detection of water in HAT-P-1b from WFC3 near-IR spatial scan observations. MNRAS, 435, 3481-3493. 1.3

Waldmann, I. P. (2012) Of "Cocktail Parties" and Exoplanets. ApJ, 747, 12. 1.4, 2.5.6

Winn, J. N., Holman, M. J., Torres, G., McCullough, P., Johns-Krull, C., Latham, D. W., Shporer, A., Mazeh, T. et al. (2008) The Transit Light Curve Project. IX. Evidence for a Smaller Radius of the Exoplanet XO-3b. ApJ, 683, 1076-1084. 2.5 .4

Winn, J. N., Johnson, J. A., Marcy, G. W., Butler, R. P., Vogt, S. S., Henry, G. W., Roussanova, A., Holman, M. J. et al. (2006) Measurement of the SpinOrbit Alignment in the Exoplanetary System HD 189733. ApJ, 653, L69-L72. 1.1.2.1

Winn, J. N., Noyes, R. W., Holman, M. J., Charbonneau, D., Ohta, Y., Taruya, A., Suto, Y., Narita, N. et al. (2005) Measurement of Spin-Orbit Alignment in an Extrasolar Planetary System. ApJ, 631, 1215-1226. 1.1.2.1

Wolszczan, A. \& Frail, D. A. (1992) A planetary system around the millisecond pulsar PSR1257 + 12. Nature, 355, 145-147. 1

Wood, P. L., Maxted, P. F. L., Smalley, B. \& Iro, N. (2011) Transmission spectroscopy of the sodium ' $\mathrm{D}$ ' doublet in WASP-17b with the VLT. MNRAS, 412, 2376-2382. 2.1

Yeredor, A. (2000) Blind separation of Gaussian sources via second-order statistics with asymptotically optimal weighting. IEEE Signal Processing Letters, 7, 197200. 2.5.6

Zhao, M., O'Rourke, J. G., Wright, J. T., Knutson, H. A., Burrows, A., Fortney, J., Ngo, H., Fulton, B. J. et al. (2014) Characterization of the Atmosphere of the 
Hot Jupiter HAT-P-32Ab and the M-dwarf Companion HAT-P-32B. ApJ, 796, 115. 3.2

Zhou, G. \& Bayliss, D. D. R. (2012) Detection of sodium absorption in WASP-17b with Magellan. MNRAS, 426, 2483-2488. 2.1

Zhou, G., Bayliss, D. D. R., Kedziora-Chudczer, L., Salter, G., Tinney, C. G. \& Bailey, J. (2014) $\mathrm{K}_{s}$-band secondary eclipses of WASP-19b and WASP-43b with the Anglo-Australian Telescope. MNRAS, 445, 2746-2757. 1.2.1 



\section{Acknowledgements}

First and foremost, I would like to thank Stefan Dreizler for his commitment during the supervision of this thesis. He was always approachable and eager to help, even at odd hours. I appreciate the freedom he gave me in perusing my thesis work, which has allowed me to take advantage of many great opportunities.

I would, further, like to thank my co-advisors Ansgar Reiners and Peter Hauschildt for their helpful feedback during the meetings of the GrK 1351 'Extrasolar Planets and their Host Stars'. I valued these meetings as they provided an informal but stimulating environment for scientific exchange between students and advisors interested in the same topic.

I would like to thank the co-authors of my papers, Enric Pallé, Felipe Murgas, Nicolas Iro, Antonio Cabrera-Lavers, Jacob Bean, Travis Barman and Stefan Dreizler for the helpful scientific discussions and their support in finishing my thesis project. I would like to give special thanks to Markus Kissler-Patig and Bob Fosbury for their mentoring during my stay at ESO at the beginning of my thesis project. Our lunch and coffee discussions have broadened my horizon and rejuvenated my curiosity.

I would like to thank Enric Pallé for his mentoring during my stays at IAC. My deepest thanks go to all the wonderful people at IAC, who have welcomed me so warmly in their midst. You have made my stays in Tenerife not only scientifically productive but also personally enriching. I will forever treasure my time on the island. At this point I would like to give special thanks to Hannu Parviainen for opening his house to me, showing me around the island and teaching me to embrace the island way of life as well as for our tremendously helpful scientific conversations. Finally, I would like to thank my friends and family for their open ears and moral support, for sharing their experiences and looking out for me. Special thanks goes to Sebastian Schnaidt for his assistance during the very last days. 



\title{
Curriculum vitae
}

\author{
Lisa Nortmann \\ Christophorusweg 14 \\ 37075 Göttingen
}

Geburtstag: 25. 01. 1987

Geburtsort: Hamburg

Nationalität: deutsch

\section{Ausbildung}

\section{Universität}

$01 / 2012-12 / 2015$

19. 12.2011

10. 08. 2009

$10 / 2006-12 / 2011$

Wissenschaftliche Mitarbeiterin, (Universität Göttingen)

Abschluss M. Sc. Physics (Universität Göttingen)

Abschluss B. Sc. Physics (Universität Göttingen)

Physikstudium, (Universität Göttingen)

\section{Schule}

26. 06. 2006

$08 / 1999-06 / 2006$

allgemeine Hochschulreife

Matthias-Claudius-Gymnasium Gehrden

$1997-1999$

Burgbergschule Gehrden (Orientierungsstufe)

$1996-1997$

Grundschule Gehrden

$1993-1996$

Schule Trenknerweg Hamburg (Grundschule) 